\title{
A Review of Possible Planetary Atmospheres in the TRAPPIST-1 System
}

\author{
Martin Turbet $^{1}$ (D) Emeline Bolmont ${ }^{1}$. \\ Vincent Bourrier $^{1}$ - Brice-Olivier Demory ${ }^{2}$. \\ Jérémy Leconte $^{3} \cdot$ James Owen $^{4} \cdot$ Eric T. Wolf $^{5}$
}

Received: 14 January 2020 / Accepted: 4 July 2020 / Published online: 23 July 2020

(C) The Author(s) 2020

\begin{abstract}
TRAPPIST-1 is a fantastic nearby ( $~ 39.14$ light years) planetary system made of at least seven transiting terrestrial-size, terrestrial-mass planets all receiving a moderate amount of irradiation. To date, this is the most observationally favourable system of potentially habitable planets known to exist. Since the announcement of the discovery of the TRAPPIST-1 planetary system in 2016, a growing number of techniques and approaches have been used and proposed to characterize its true nature. Here we have compiled a stateof-the-art overview of all the observational and theoretical constraints that have been obtained so far using these techniques and approaches. The goal is to get a better understanding of whether or not TRAPPIST-1 planets can have atmospheres, and if so, what they are made of. For this, we surveyed the literature on TRAPPIST-1 about topics as broad as irradiation environment, planet formation and migration, orbital stability, effects of tides and Transit Timing Variations, transit observations, stellar contamination, density measurements, and numerical climate and escape models. Each of these topics adds a brick to our understanding of the likely — or on the contrary unlikely_atmospheres of the seven known planets of the system. We show that (i) Hubble Space Telescope transit observations, (ii) bulk density measurements comparison with $\mathrm{H}_{2}$-rich planets mass-radius relationships, (iii) atmospheric escape modelling, and (iv) gas accretion modelling altogether offer solid evidence against the presence of hydrogen-dominated—cloud-free and cloudy-atmospheres around
\end{abstract}

Understanding the Diversity of Planetary Atmospheres

Edited by François Forget, Oleg Korablev, Julia Venturini, Takeshi Imamura, Helmut Lammer and Michel Blanc

\section{Turbet}

1 Observatoire Astronomique de l'Université de Genève, 51 chemin de Pégase, 1290 Sauverny, Switzerland

2 Center for Space and Habitability, University of Bern, Gesellschaftsstrasse 6, 3012, Bern, Switzerland

3 Laboratoire d'astrophysique de Bordeaux, Univ. Bordeaux, CNRS, B18N, allée Geoffroy Saint-Hilaire, 33615 Pessac, France

4 Astrophysics Group, Department of Physics, Imperial College London, Prince Consort Rd, London, SW7 2AZ, UK

5 Laboratory for Atmospheric and Space Physics, University of Colorado, Boulder, CO 80309, USA 
TRAPPIST-1 planets. This means that the planets are likely to have either (i) a high molecular weight atmosphere or (ii) no atmosphere at all. There are several key challenges ahead to characterize the bulk composition(s) of the atmospheres (if present) of TRAPPIST-1 planets. The main one so far is characterizing and correcting for the effects of stellar contamination. Fortunately, a new wave of observations with the James Webb Space Telescope and nearinfrared high-resolution ground-based spectrographs on existing very large and forthcoming extremely large telescopes will bring significant advances in the coming decade.

Keywords TRAPPIST-1 $\cdot$ Exoplanets $\cdot$ Atmospheres $\cdot$ Review

\section{Introduction}

Nearly 25 years after the first detection of an exoplanet orbiting a solar-type star (Mayor and Queloz 1995), several thousand extrasolar planets have been detected at a frenetic rate (Schneider et al. 2011; Akeson et al. 2013). While the science of exoplanets initially focused mainly on the detection of exoplanets, it is gradually moving towards their characterisation. A large number of space missions (e.g. Hubble Space Telescope, James Webb Space Telescope, ARIEL) and ground-based instruments (e.g. HARPS, VLT-ESPRESSO, ELT-HIRES) mounted on large telescopes are in the process of thoroughly characterizing the atmospheric composition, chemistry, clouds, and many other properties of large warm exoplanets which are the most amenable for in-depth characterization. This opens up the field of comparative exoplanetology. To a lesser extent, the detection rate of small possibly temperate exoplanets, much more difficult to observe, has also exploded in recent years. Nearly 40 exoplanets with a mass and/or radius similar to that of the Earth, and with incident fluxes close to that received on Earth, have been detected so far. However, the vast majority of these planets are inaccessible to our telescopes for the characterization of their atmosphere and surface. The TRAPPIST-1 system - at the heart of this review-provides us with a natural laboratory to characterize for the first time, in a few years only, the atmospheres and surfaces of temperate rocky planets outside the solar system. The exploration of TRAPPIST-1 is likely to revolutionize, through comparative planetology, all the knowledge we have accumulated so far about the evolution of the atmospheres and habitability of terrestrial planets.

TRAPPIST (the TRansiting Planets and PlanestIsimals Small Telescope; Gillon et al. 2011, 2013), a small 60-cm ground-based telescope located at the ESO La Silla Observatory in Chile, monitored the brightness of the star 2MASS J23062928-0502285 (a.k.a. EPIC 246199087, or simply TRAPPIST-1) for 245 hours over 62 nights from 17 September to 28 December 2015. The analysis of the light curves measured during these observation series (Gillon et al. 2016) led to the detection of two transit-like signatures with amplitudes close to $1 \%$ named TRAPPIST-1b and $\mathrm{c}$, and a tentative detection of a third planet for which the orbital period was not known. Starting 19 September 2016, nearly 20 days of quasi-continuous photometric monitoring with NASA's Spitzer Space Telescope ${ }^{1}$ identified that the third signal measured by TRAPPIST was in fact a combination of multiple signals due to the presence of several additional planets in the system (Gillon et al. 2017), named TRAPPIST-1d, e, f and g. These observations also led to the detection of an orphan transit, indicating the possible presence of a seventh planet in the system, named TRAPPIST- $1 \mathrm{~h}$.

\footnotetext{
${ }^{1}$ This photometric campaign was complemented by TRAPPIST (South and North), LT/IO:O, UKIRT/WFCAM, WHT/ACAM, SAAO-1m/SHOC, VLT/HAWK-1, HCT/HFOSC and HST/WFC3 observations, listed by decreasing number of hours of observation (Gillon et al. 2017).
} 
The existence of this seventh planet was later confirmed (Luger et al. 2017b) through a 79 consecutive days observation campaign (starting 15 December 2016) with the NASA's Kepler Space Telescope in its two-reaction wheel mission (a.k.a. K2; Howell et al. 2014). Follow-up observations (Delrez et al. 2018; Ducrot et al. 2018; Burdanov et al. 2019; Ducrot et al. 2020) later not only confirmed the existence of at least seven temperate, terrestrial-size planets around the star TRAPPIST-1, but also helped to better constrain their main properties (summarized in Table 1).

The TRAPPIST-1 system is exceptional because it is - through a series of techniques that will be discussed in this review paper-the most observationally favourable system of potentially habitable planets (i.e. planets that could have liquid water on their surface and can therefore have the preconditions for life as we know it on Earth) known to exist. This mostly results from a subtle combination of (i) its proximity (39.14 light years from us), (ii) the fact that planets are transiting (frequently) in front of their star, and (iii) the extremely small radius of the ultra-cool dwarf host star TRAPPIST-1.

Since the discovery of the TRAPPIST-1 system was announced in 2016 (Gillon et al. 2016), a flourishing number of multidisciplinary scientific works have been carried out (about 170 peer-reviewed publications per year in 2018 and 2019; source: NASA/ADS) to obtain information on the true nature of the TRAPPIST-1 system. The main purpose of this review is to set the stage of what we think we have learned so far about this system and what this implies for the presence and nature (if any) of TRAPPIST-1 planetary atmospheres. The second purpose of this review is to discuss the future opportunities available-with increasingly large telescopes and increasingly performant instruments - to characterize the nature of the TRAPPIST-1 planets, particularly through their atmosphere, and to identify the potential challenges that lie ahead.

Firstly, we review in Sect. 2 previous works on the stellar environment (irradiation, stellar activity) in order to identify the context in which the planets of the TRAPPIST- 1 system and their atmospheres have evolved. Secondly, we present in Sect. 3 previous works carried out on the orbital architecture of the system, which contains key information on (1) how the planets were formed (including how much volatile they accreted in the first place), (2) their mode of rotation and (3) their masses. These are three key pieces of information for interpreting the nature of the TRAPPIST-1 planets and their possible atmospheres. Thirdly, we gather and then discuss in Sect. 4 all existing multi-wavelengths transit observations (with HST, Spitzer, K2 and ground-based telescopes) of TRAPPIST-1 planets. These observations can not only help us to eliminate a number of hypotheses about the compositions of TRAPPIST-1 planetary atmospheres, but also to identify key challenges for their spectroscopic characterization with the future generation of large telescopes. Fourthly, we review in Sect. 5 the theoretical and numerical advances that have been made-using sophisticated numerical atmospheric and escape models - in recent years on the atmospheres of planets orbiting ultra-cool stars, and what this implies for the range of possible compositions of planetary atmospheres in the TRAPPIST-1 system. Fifthly, we provide in Sect. 6 an overview of the near and far-future prospects to detect and characterize (if present) these atmospheres. While there are many challenges ahead, the prospects for future characterization are extremely promising. Finally, the most important conclusions of this review are summarized in Sect. 7.

\section{Constraints from the Stellar Environment}

A fundamental characteristic of the TRAPPIST-1 planets is that they orbit a very small, very cold and very low mass star. The evolution of the luminosity and the activity of such stars 


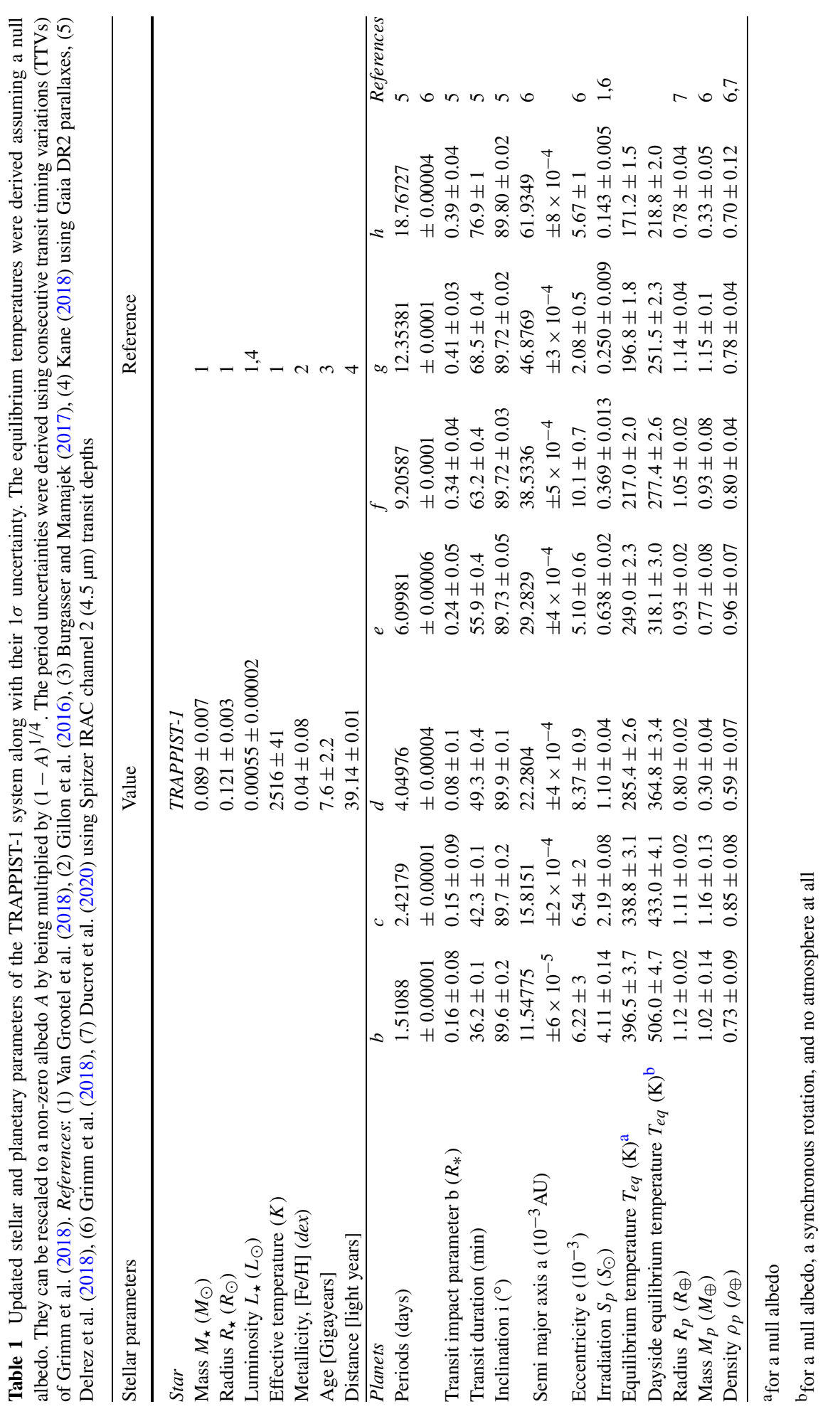




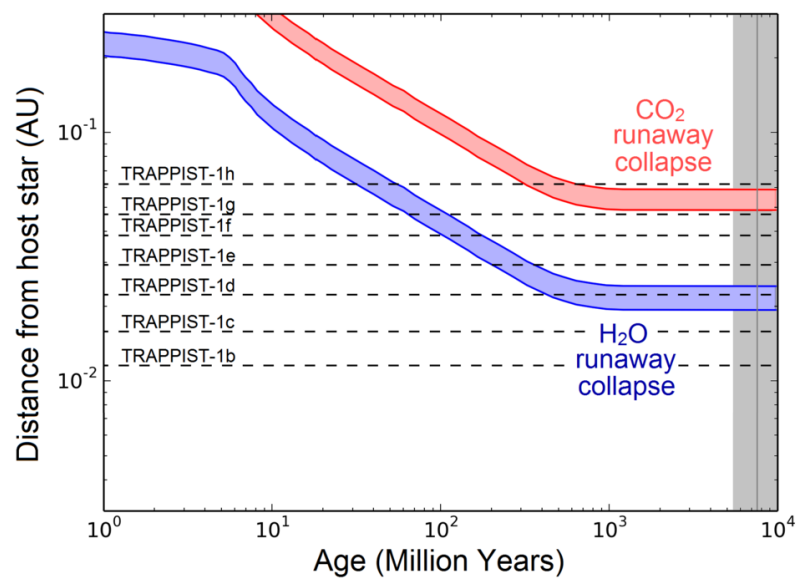

Fig. 1 Architecture of the TRAPPIST-1 system and evolution of the runaway greenhouse/atmospheric collapse limit for water (a.k.a. the traditional inner edge of the Habitable Zone) and carbon dioxide. The spread of the runaway greenhouse/atmospheric collapse for water was calculated assuming a synchronous planet (i.e. at $1.4 \times$ the bolometric flux received on Earth; see Yang et al. 2014a.) and a non-synchronous planet (i.e. at $0.9 \times$ the bolometric flux received on Earth, using the results of the 1-D calculations of Kopparapu et al. 2013b,a). Note that the $1.4 \times \mathrm{F}_{\oplus}$ limit is a conservative estimate according to the results of Kopparapu et al. 2016 showing this threshold could vary depending on the metallicity of the host star, because the stellar mass-luminosity relationship depends on the metallicity, and thus does the rotation rate of the planet. Moreover, this runaway greenhouse estimate has been calculated assuming a cold start which is likely not a good approximation for planets orbiting such ultra-cool dwarfs, and that are thought to have started hot. The spread of the runaway greenhouse/atmospheric collapse for $\mathrm{CO}_{2}$ was calculated based on the results of Turbet et al. (2018) that the irradiation limit at which $\mathrm{CO}_{2}$ cannot accumulate in the atmosphere of TRAPPIST-1 planets is located between the orbit of TRAPPIST-1g and $\mathrm{h}$. This result is discussed in more details in the Sect. 5.6 of the manuscript. These lines were drawn assuming a luminosity derived from evolutionary models for a $0.089 M_{\odot}$ M-dwarf (Van Grootel et al. 2018). For reference, we added the estimated age of $7.6 \pm 2.2$ Gigayears based on Burgasser and Mamajek (2017). The figure was adapted from Bourrier et al. (2017a)

have severe consequences on the evolution of planetary atmospheres, which we review in this section.

\subsection{Temporal Evolution of TRAPPIST-1 Luminosity and Runaway Greenhouse}

Ultra-cool stars such as TRAPPIST-1 can stay for hundreds of millions of years in the Pre Main Sequence (PMS) phase, a phase during which their luminosity can decrease possibly by several orders of magnitude (Chabrier and Baraffe 1997; Baraffe et al. 1998, 2015). During this PMS phase, planets are exposed to strong irradiation, which make them very sensitive to atmospheric processes such as hydrodynamical escape (Vidal-Madjar et al. 2003; Lammer et al. 2003) or runaway greenhouse (Ramirez and Kaltenegger 2014), indicating that all the common-so-called volatile-molecular species (e.g. $\mathrm{H}_{2} \mathrm{O}, \mathrm{SO}_{2}, \mathrm{NH}_{3}, \mathrm{CO}_{2}$ ) and most of their byproducts must be in gaseous form in the atmosphere.

As an illustration, and following Bolmont et al. (2017a) and Bourrier et al. (2017a), Fig. 1 shows how the limit at which all water (in blue) should be vaporized in a planetary atmosphere, as a function of time. In other words, for planets located closer to TRAPPIST-1 than the blue curve, water is expected to be unstable in condensed (solid or liquid) form and should form a steam atmosphere. This limit is also known as the runaway greenhouse (Ingersoll 1969; Kasting 1988; Pierrehumbert 2010; Goldblatt and Watson 2012). 
First, Fig. 1 illustrates the fact that TRAPPIST-1b, c, and also maybe TRAPPIST-1d, ${ }^{2}$ have spent their entire life in a state where water can only be present in the form of steam. Second, the four outermost planets of the system are compatible with the presence of surface water in liquid or icy form today, but all four planets must have spent a significant fraction of their lives in a state where all water was trapped in vapour form in the atmosphere.

For illustration, and based on the results of Turbet et al. (2018), we added in Fig. 1 the limit (in red) at which all available $\mathrm{CO}_{2}$ should be vaporized in a planetary atmosphere (or collapse on the surface, respectively), as a function of time. The conditions required for atmospheric collapse were studied in depth with numerical and analytical models (Joshi et al. 1997; Wordsworth 2015; Koll and Abbot 2016; Auclair-Desrotour and Heng 2020) and applied specifically to the TRAPPIST-1 system in Turbet et al. (2018). The only planets of the system sensitive to strong $\mathrm{CO}_{2}$ atmospheric collapse today are TRAPPIST-1g and h. $\mathrm{CO}_{2}$ collapse can theoretically occur on the other planets but it requires some special conditions (Turbet et al. 2018) discussed in Sect. 5.6 and Fig. 9, the main condition being that the planets must be depleted of non-condensable gases. Other common gases such as $\mathrm{CH}_{4}, \mathrm{O}_{2}$, $\mathrm{CO}$ or $\mathrm{N}_{2}$ are too volatile to be sensitive to atmospheric collapse on the TRAPPIST-1 planets (Turbet et al. 2018). If present, these volatile species should be in the atmosphere (i.e. not trapped on the surface).

\subsection{Stellar Activity and Atmospheric Loss}

Knowing the XUV (i.e. from X to UV) irradiation of TRAPPIST-1 is crucial because it affects the stability and erosion of planetary atmospheres (Lammer et al. 2003; Bolmont et al. 2017a), controls photochemical reactions in the upper atmosphere (Rugheimer et al. 2015a; Arney et al. 2017; Chen et al. 2019), and can further influence the development and survival of life on a planet surface (Rugheimer et al. 2015b; O'Malley-James and Kaltenegger 2017; Ranjan et al. 2017). As a M8-type star, TRAPPIST-1 is thought to be a very active star, with a strong X/Extreme UV (EUV) flux (Wheatley et al. 2017; Bourrier et al. 2017a) and frequent, intense flaring events (Vida et al. 2017). However, little is known about the FUV emission of these ultra-cool dwarfs. In fact TRAPPIST-1 is the coldest exoplanet host star for which FUV emission has been measured, via measurement of its Lyman- $\alpha$ line with HST/STIS (Bourrier et al. 2017b). The comparison between this measurement and that of TRAPPIST-1 X-ray emission (Wheatley et al. 2017) further show that the stellar chromosphere is only moderately active compared to its transition region and corona.

Based on (i) HST/STIS Lyman- $\alpha$ observations of TRAPPIST-1 (Bourrier et al. 2017b,a), (ii) XMM-Newton X-ray observations of TRAPPIST-1 (Wheatley et al. 2017), (iii) constraints from GALEX far-UV and mid-UV photometry survey (partly based on the work of Schneider and Shkolnik 2018) on a sample of very nearby, similar late (M8) stars, and (iv) PHOENIX Models, ${ }^{3}$ Peacock et al. (2019) constructed full emission spectra of TRAPPIST-1 from $X$ to far-infrared wavelengths. Figure 2 shows a calculated emission spectrum of TRAPPIST-1 (Peacock et al. 2019), normalized to a total bolometric flux of $1366 \mathrm{~W} \mathrm{~m}^{-2}$, i.e. the mean irradiation received at the top of the atmosphere of present-day Earth. This corresponds to the stellar flux that an hypothetical planet located at $\sim 0.023 \mathrm{AU}$ of the star TRAPPIST-1 (between the orbits of planet $\mathrm{d}$ and e) would receive. Black data points correspond (by increasing wavelength) to:

\footnotetext{
${ }^{2}$ Whether or not TRAPPIST-1d is below or beyond the runaway greenhouse irradiation limit for water depends on subtle cloud and atmospheric circulation feedbacks (Yang et al. 2013, 2014b; Kopparapu et al. 2016; Wolf 2017; Turbet et al. 2018).

3 available on https://phoenix.ens-lyon.fr/simulator/index.faces.
} 


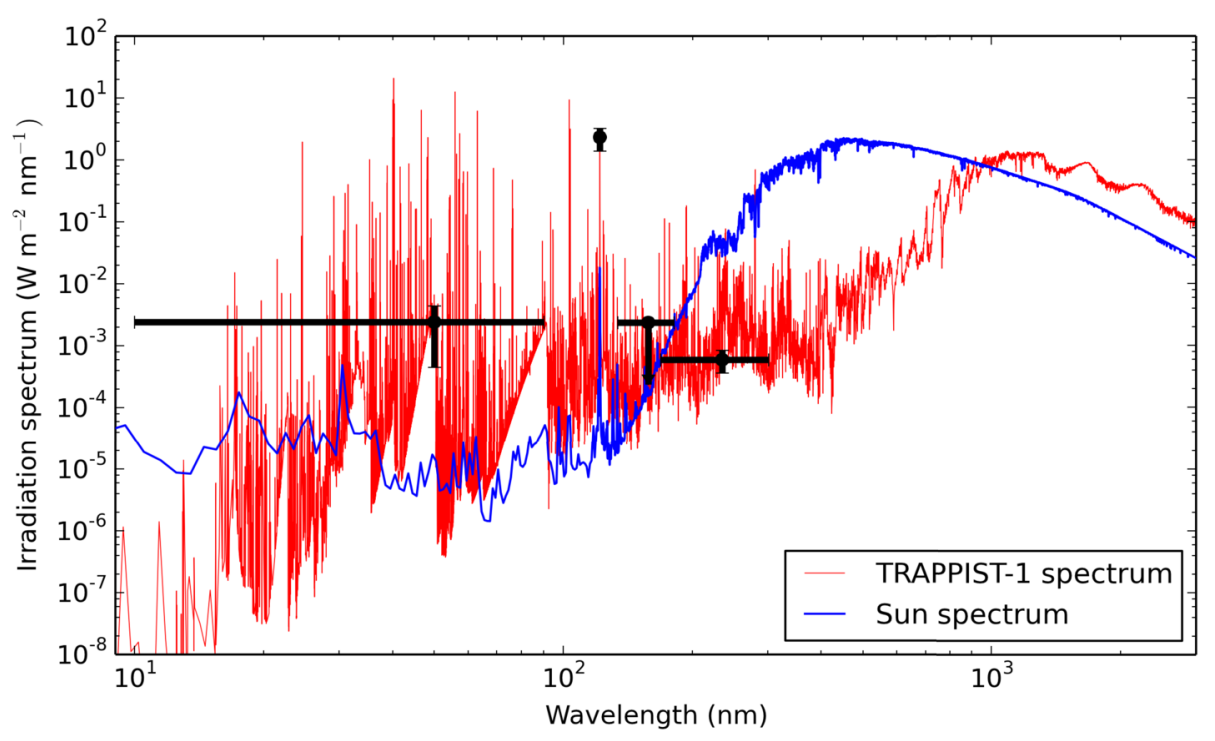

Fig. 2 This figure shows irradiation spectra emitted by the star TRAPPIST-1 (red line) and the Sun (blue line). Both spectra were normalized to a total bolometric flux of $1366 \mathrm{~W} \mathrm{~m}^{-2}$, i.e. the mean irradiation received at the top of the atmosphere of present-day Earth. The solar spectrum (blue line) is the solar reference spectrum (SOLAR-ISS) taken from Meftah et al. (2018). The TRAPPIST-1 spectrum (red line) is calculated in Peacock et al. (2019) (scenario 1A). Black data points are described in the main text (Sect. 2.2)

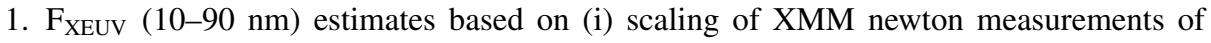
X-ray emission of TRAPPIST-1 (Wheatley et al. 2017) into EUV emission using the $\mathrm{F}_{\mathrm{EUV}} / \mathrm{F}_{\mathrm{X}}$ scaling relationship of Chadney et al. (2015); (ii) scaling of Ly $\alpha$ line measurements of TRAPPIST-1 using HST/STIS observations (Bourrier et al. 2017b,a) into EUV emission using the $\mathrm{F}_{\mathrm{EUV}} / \mathrm{F}_{\mathrm{Ly} \alpha}$ scaling relationship of Linsky et al. (2014), summed with the observed X-ray flux of Wheatley et al. (2017).

2. Ly $\alpha$ line (121.44-121.7 nm) measurements based on HST/STIS observations of TRAPPIST-1 (Bourrier et al. 2017b,a). Note that the synthetic spectrum of TRAPPIST-1 (red line) was plotted at very high resolution between 121.44 and $121.7 \mathrm{~nm}$ to make the comparison with the HST/STIS data easier.

3. GALEX FUV (134.0-181.1 nm) upper estimate measurements of the emission of three very nearby M8-M8.5 very low mass stars (2MASS 12590470-4336243, 2MASS 10481463-3956062, 2MASS 18353790+3259545), i.e. that are of a spectral type similar to TRAPPIST-1. The three stars have an estimated age of $~ 5$ Gigayears (Schneider and Shkolnik 2018; Peacock et al. 2019), which is within a few gigayears of the estimated age of TRAPPIST-1 (Burgasser and Mamajek 2017).

4. GALEX NUV (168.7-300.8 $\mathrm{nm}$ ) measurements of the emission of the same three very nearby M8-M8.5 very low mass stars.

Note that the infrared part of the calculated TRAPPIST-1 spectrum of Peacock et al. (2019) (red line) is consistent with the IRTF/SpeX near-infrared measurements of Gillon et al. (2016).

Figure 2 shows that while the thermal emission of TRAPPIST-1 ( $\left.T_{\text {eff }} \sim 2516 \mathrm{~K}\right)$ is shifted to higher wavelength than the Sun $\left(T_{\text {eff }}=5778 \mathrm{~K}\right)$, the non thermal emission of TRAPPIST-1 is significantly higher than that of the Sun (relative to the total bolometric 
flux) for wavelengths lower than $150 \mathrm{~nm}$. In other words, at constant bolometric flux, the $\mathrm{X}$-EUV and more energetic photon flux is much larger for TRAPPIST-1 than Sun-like stars. Moreover, this strong high-energy photon flux was likely much higher in the past (Bourrier et al. 2017a; Fleming et al. 2020). In fact, because the XUV irradiation is expected to decrease faster with time than the bolometric irradiation, the relative fraction of XUV irradiation was likely even much larger than today. This is an important aspect because it indicates that the atmospheric escape processes were likely much more efficient in the TRAPPIST-1 system than in the solar system. This energetic photon flux is indeed likely to drive strong atmospheric escape, possibly hydrodynamically-driven (Roettenbacher and Kane 2017; Bourrier et al. 2017a). Repeated measurements of TRAPPIST-1 Lyman- $\alpha$ line with HST/STIS show that the stellar UV emission varies over timescales of a few months (Bourrier et al. 2017b,a), suggesting similar variability in the strength of atmospheric escape and highlighting the need for long-term monitoring of the system.

Furthermore, flaring events likely add an additional, significant component to the high-energy emission of TRAPPIST-1. Vida et al. (2017) measured using K2 data that TRAPPIST-1 frequently produces flaring events of integrated intensities ranging from $1.26 \times 10^{23}$ to $1.24 \times 10^{26} \mathrm{~J}$. As a reference, the energy brought by the most intense flaring event reported by Vida et al. (2017) corresponds to the integrated bolometric emission of TRAPPIST-1 during $\sim 10$ minutes. For comparison, the most intense known solar flares have an integrated intensity $\sim 10^{25} \mathrm{~J}$ (Shibata and Magara 2011), which corresponds to the integrated bolometric emission of the Sun during $\sim 0.03$ second. While flares can have a substantial effect on atmospheric erosion, and possibly even on photochemistry (Segura et al. 2010), they should have a minimal effect on the direct warming of the surface and atmosphere of TRAPPIST-1 planets.

In addition, atmospheric stripping by the strong stellar winds of TRAPPIST-1 is thought to be efficient (Garraffo et al. 2017; Dong et al. 2017, 2018, 2019; Fraschetti et al. 2019) for planets orbiting such a low mass star.

Consequently, it is likely that the planets of the TRAPPIST- 1 system all have lost a significant fraction of their initial atmosphere, and may possibly have completely lost it. The fact that the planets of the TRAPPIST-1 system today have an atmosphere or not results from a competition between (i) the efficiency and duration of the atmospheric escape processes and (ii) the amount of volatiles (i.e., which can form an atmosphere; this can for example be water, carbon dioxide, methane, nitrogen, etc.) initially present on the planet and later brought in by degassing and by cometary or asteroid impacts.

\section{Constraints from the Orbital Architecture of the TRAPPIST-1 Planetary System}

The orbital architecture of the TRAPPIST-1 planetary system is very peculiar (see Fig. 3). First, it is extremely compact. All seven planets are confined within $\sim 0.06$ AU from their host star (Luger et al. 2017b). Secondly, all planets have a highly circularized orbit, with eccentricities lower than 0.01 for all planets (Gillon et al. 2017; Grimm et al. 2018). Thirdly, the system is very coplanar (Luger et al. 2017a; Delrez et al. 2018). Last but not least, all the planets form a resonant chain, and therefore have strong mutual gravitational interactions. Each pair of adjacent planets (bc, cd, de, ef, fg, gh) have period ratios near small integer ratios, and each triplets of adjacent planets (bcd, cde, def, efg, fgh) follow three-body Laplace resonances (Luger et al. 2017b). We discuss below how we can take advantage of this peculiar orbital architecture (i) to infer the formation and migration history of the TRAPPIST-1 

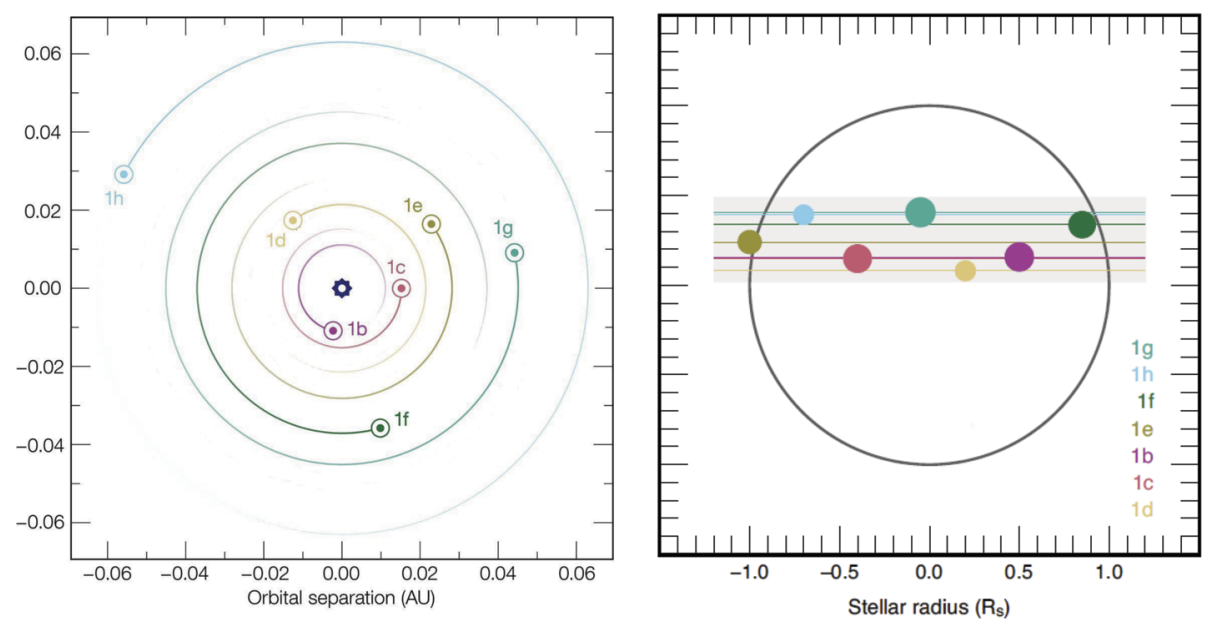

Fig. 3 Representation of the TRAPPIST-1 system viewed from above (left panel, figure adapted from Luger et al. 2017b) or seen edge-on with the seven planets transiting in front of their star (right panel; figure taken from Delrez et al. 2018)

system, (ii) to infer masses of the planets, first through orbital stability analysis, then through Transit Timing Variations (TTVs) analysis, and (iii) to predict the possible rotational states of TRAPPIST-1 planets, all three of which have implications on the possible atmospheres of TRAPPIST-1 planets today.

\subsection{Formation and Migration Scenarios for the TRAPPIST-1 System}

In the initial discovery papers (Gillon et al. 2017; Luger et al. 2017b) as well as in following discussions on the system's formation and evolution (Ormel et al. 2017; Tamayo et al. 2017; Papaloizou et al. 2018; Coleman et al. 2019; Schoonenberg et al. 2019; Brasser et al. 2019), it has been proposed that the TRAPPIST-1 planets had to undergo migration to end up being locked into resonances. Specifically, several authors (Ormel et al. 2017; Coleman et al. 2019) have argued that the most likely formation scenario for the TRAPPIST-1 system is (i) planets formed far away from their host star, likely exterior to the water ice line and (ii) planets migrated inwards (in a timescale of $\sim 10^{6}$ years) in resonant convoys to reach their present location, very close to their host star. The inner edge of the disk would provide a migration barrier (Masset et al. 2006) such that the planets pile up into chains of mean motion resonances (Terquem and Papaloizou 2007; Ogihara and Ida 2009; Cossou et al. 2014; Izidoro et al. 2017).

Meanwhile, MacDonald and Dawson (2018) argued that long-distance migration is not the only plausible explanation for the formation of the TRAPPIST-1 resonant chain. In fact, they showed using orbital numerical simulations that if TRAPPIST-1 planets have formed (quasi) in situ, then either short-distance migration or eccentricity damping could have naturally lead the system toward a resonant chain similar to TRAPPIST-1 system.

Whether TRAPPIST-1 planets formed in situ or beyond the ice line has severe consequences regarding the amount of volatile species (e.g. water) that the planets were able to accrete in the first place. If planets formed in situ, then planets are likely dry today due to strong atmospheric erosion; if planets formed beyond the ice line, then planets are likely 
volatile-rich (and water-rich) because even atmospheric erosion should be insufficient to remove $>1-10 \%$ of the total planetary mass in volatile for these planets (Tian and Ida 2015; Bolmont et al. 2017a; Bourrier et al. 2017a).

In fact, even if the first scenario-in which planets formed far from their host star and then migrated inward-is correct, it is possible that the volatile content remains very different between the inner and outer planets of the system. First, it is possible that the inner and outer planets have migrated in several distinct groups (Papaloizou et al. 2018) — that merged afterward - and have thus been formed at different locations of the protoplanetary disk, with different bulk compositions. Then, it is possible that the seven planets were each formed mainly from planetesimal accretion or pebble accretion (Coleman et al. 2019; Schoonenberg et al. 2019) which would lead to a scatter in TRAPPIST-1 planets volatile bulk composition.

Last but not least, even if the planets formed in situ and all volatiles on the surface and atmosphere were stripped through atmospheric erosion, secondary outgassing or latestage volatile delivery could still have been brought through cometary or asteroid impacts (Kral et al. 2018; Dencs and Regály 2019). Impact volatile delivery holds mostly for outer TRAPPIST-1 planets, for which impactor velocities are expected to be low enough that volatile delivery dominates over impact erosion mechanisms (Kral et al. 2018).

We note there are some ongoing efforts to characterize the outer part of the TRAPPIST-1 system (Marino et al. 2020) to better constrain the whole architecture of the system and possibly look for exocometary or exoasteroid belts.

\subsection{Stability of the TRAPPIST-1 System}

The fact that the TRAPPIST-1 system is observed today with its near-integer period ratios after $\sim 8$ billion years (Burgasser and Mamajek 2017) suggests that the orbital architecture of the system is long-lived.

Despite this apparent long-term stability, initial N-body simulations aimed at reproducing the TRAPPIST-1 system (Gillon et al. 2017) were unstable on a very short timescale ( $\sim 0.5$ million years) even when including the eccentricity damping effect of tides (which only delayed the instability by a few million years).

In contrast, Tamayo et al. (2017) prepared N-body simulations of various planetary systems similar to TRAPPIST-1 and let them evolve through disk migration to form resonant chains of planets. They found that, even without accounting for tidal dissipation, most physically plausible resonant chains of planets were stable on timescales of at least $50 \mathrm{Myr}$, i.e. two orders of magnitude larger than in Gillon et al. (2017). This result shows that when a TRAPPIST-1-like resonant chain of terrestrial-mass planets is formed, it is generally very stable over time (Tamayo et al. 2017). However, the exact stable orbital configuration of a resonant planet chain depends strongly on the parameters (orbital periods, masses, radii, eccentricities, etc.) of the planets. This indicates that the stability of a given observed resonant chain of planets such as TRAPPIST-1 is highly dependent on the initial planet parameters assumed. Therefore, it is likely that the N-body simulations of Gillon et al. (2017) were unstable because the selected planet properties of the planets were far enough from reality.

Quarles et al. (2017) took advantage of this result to estimate the masses of TRAPPIST-1 planets. For this, they performed thousands of N-body simulations of TRAPPIST-1 with planet properties perturbed from the observed values and then identified those that were stable for millions of years. With this stability analysis, Quarles et al. (2017) identified selfconsistent orbital solutions (i.e. that are stable on the long-term) from which they derived a posterior distribution of masses for each of the seven TRAPPIST-1 planets. Theses masses are provided in Table 2. Makarov et al. (2018) confirmed-using the planet properties of Quarles et al. (2017) — that the stability of the system was greatly improved. 
Table 2 Estimates of TRAPPIST-1 planet masses derived using (i) stability analysis, and (ii) TTV analysis. TTV masses and stability masses are all compatible within $1 \sigma$. However, the TTV estimates are much more precise than the stability ones. Stability masses were derived from Quarles et al. (2017) $^{\mathrm{a}}$, while TTV masses were derived from Grimm et al. $(2018)^{\mathrm{b}}$. Mass estimates are provided in Earth mass units, and with $1 \sigma$ uncertainties

Fig. 4 Measured transit times of TRAPPIST-1c (with corresponding $1 \sigma$ uncertainties) are indicated by coloured symbols, according to the origin of the data (Spitzer, K2 or other telescopes). The grey line indicates the spread of TTV fits obtained for one thousand distinct MCMC calculations samples (Grimm et al. 2018). The low-frequency TTV component is visible in the top panel, and the high-frequency (chopping) TTV component is visible in the bottom panel. A detailed list of all transits is given in the appendix of Grimm et al. (2018). This figure was adapted from Fig. 2 of Grimm et al. (2018), which also shows the TTVs of the 6 other planets in the system

\begin{tabular}{llc}
\hline Planet & Stability ${ }^{\mathrm{a}}$ masses & $\mathrm{TTV}^{\mathrm{b}}$ masses \\
\hline T1b & $0.88_{-0.53}^{+0.62}$ & $1.017_{-0.143}^{+0.154}$ \\
T1c & $1.35_{-0.59}^{+0.61}$ & $1.156_{-0.131}^{+0.142}$ \\
T1d & $0.42_{-0.21}^{+0.25}$ & $0.297_{-0.035}^{+0.039}$ \\
T1e & $0.55_{-0.35}^{+0.51}$ & $0.772_{-0.075}^{+0.079}$ \\
T1f & $0.68_{-0.18}^{+0.17}$ & $0.934_{-0.078}^{+0.080}$ \\
T1g & $1.39_{-0.69}^{+0.76}$ & $1.148_{-0.095}^{+0.098}$ \\
T1h & $0.47_{-0.26}^{+0.26}$ & $0.331_{-0.049}^{+0.056}$ \\
\hline
\end{tabular}
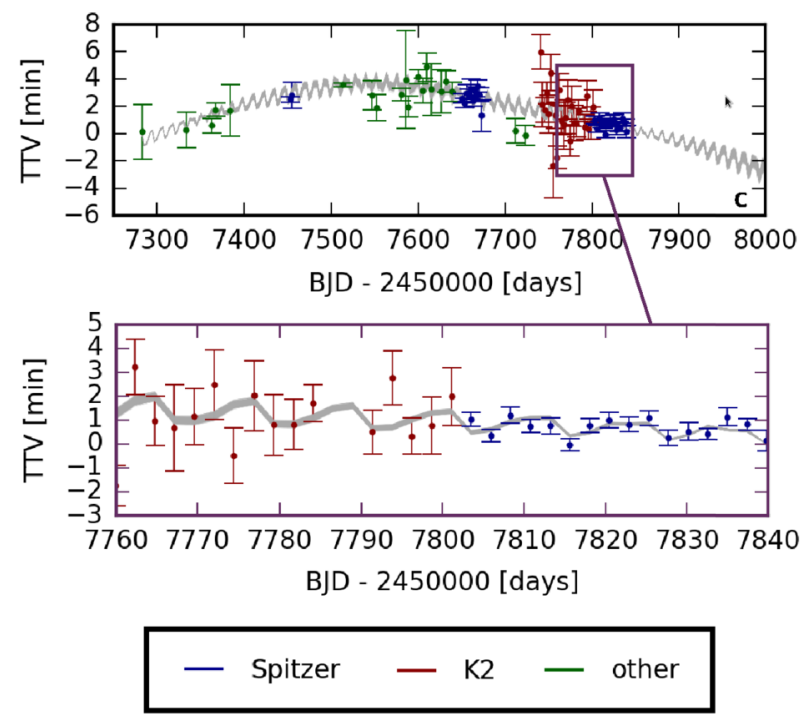

\subsection{Transit Timing Variations}

In tightly packed planetary systems such as TRAPPIST-1, the continuous exchange of angular momentum between gravitationally interacting planets causes them to accelerate and decelerate along their orbits. This makes in turn their transit times occur early or late compared with a Keplerian orbit, possibly in a detectable way. Detecting these changes in transit times is known as the Transit Timing Variations (TTV) technique (Holman and Murray 2005; Agol et al. 2005).

All TRAPPIST-1 planets exhibit transit timing variations (Gillon et al. 2017; Grimm et al. 2018) owing to gravitational pulls by their closest neighbours. The TTV signal for each planet is dominated primarily by interactions with adjacent planets, and these signals have the potential to be particularly large because each planet is near first-order mean motion resonance with its neighbours. In the TRAPPIST-1 system, the TTV amplitudes range in magnitude from 2 min to 1 hour (Gillon et al. 2017; Grimm et al. 2018) depending on the planet. 
To get accurate TTV data, it is necessary to derive precise timings for the transits of the planets. This requires transit observations with a large aperture telescope (to get as much photons as possible) and a stable photometry, to get the best possible time-resolved SNR of the transit light curve and thus the transit timing. TTVs have two components:

1. a low-frequency component, visible in the upper panel of Fig. 4. To capture this TTV component, regular transit observations are needed over several years.

2. a high-frequency component-aka chopping (Holman et al. 2010; Deck and Agol 2015)_visible in the lower panel of Fig. 4. The periodicity of chopping pattern encodes the timespan between successive conjunctions of pairs of successive planets whose amplitudes yield the masses of adjacent perturbing planets. To capture this TTV component, continuous transit observations are needed over several tens of days.

For the TRAPPIST-1 system, the most accurate TTV datasets were obtained with Spitzer (Spitzer Proposals ID 13067, 13175, 14223) space mission (see Fig. 4). However, transit observations with ground-based telescopes were also useful to constrain the long-term (or low-frequency) component of the TTV.

TTV datasets were then analyzed through inversion, a process through which observed transit times are fit using a model of gravitationally interacting planets in order to determine the system parameters (Carter et al. 2012). This specifically includes the determination of planetary masses, relative to the mass of the star. TRAPPIST-1 TTVs were first modeled in Gillon et al. (2017) and then completed in Grimm et al. (2018), for which a much larger amount of TTV data (a total of 284 individual transit timings) has been included. Grimm et al. (2018) used a sophisticated algorithm to compare and fit the outcome of a large number of N-body simulations of the TRAPPIST-1 system-initialized with a wide range of planet parameters-with the TTV data. Using this methodology, they were able to derive masses estimates for the TRAPPIST-1 planets. Theses masses estimates are provided in Table 2. TTVs masses and Stability Analyses masses are all compatible within $1 \sigma$ error bars. However, we recommend the use of TTVs masses which have a much better measurement accuracy. These masses can be used (see Sect. 5) to evaluate the planet bulk densities and gather information on their possible atmospheres. We note that significant efforts are currently being made to use all observational data-including all the latest Spitzer observation campaigns - to estimate the masses of the TRAPPIST-1 planets through TTVs as accurately as possible (Agol et al. 2020, in preparation).

\subsection{Effect of Tides on TRAPPIST-1 Planets}

All observed TRAPPIST-1 planets are inside an orbital distance of $\sim 0.06$ AU. For such close-in planets, tidal interactions are expected to be strong and to influence the orbital and rotational dynamics of the system.

In fact, the TRAPPIST-1 system principally evolves due to the gravitational tides raised by the star in the planets (the planetary tide). Simple order of magnitude calculations show that the tides exerted by the planets in the star are today a priori negligible (Turbet et al. 2018). Moreover, the contribution of tidal torques between planets has been shown to be overall very small (Wright 2018; Hay and Matsuyama 2019), compared to the planetary tides. Planetary tides act to (i) slow down the rotation rate of the planets, (ii) reduce their obliquity, and (iii) circularize their orbits. It can trap the planets into spin-orbit resonances, possibly down to the synchronous rotation.

Turbet et al. (2018) estimated the evolution timescales for the rotation to range from $10^{-4}$ Myr for TRAPPIST-1b to 7 Myr for TRAPPIST-1h. For the obliquity, the evolution 
timescales range from $10^{-3}$ Myr for planet-b to $80 \mathrm{Myr}$ for planet-h. These timescales, computed assuming a tidal dissipation for the planets to be a tenth of the dissipation of the Earth-i.e. close to an ocean-less earth -, are highly uncertain. However, considering the estimated age of the system of $\sim 8$ Gigayear (Burgasser and Mamajek 2017), it is reasonable to assume that all planets have reached a state of near tidal equilibrium, with small obliquities and a slow rotation. The exact rotation will depend on the presence and strength of other processes able to balance the braking effect of tides.

Indeed, it is now known that some other processes can sometimes act to avoid the synchronous state. A first possibility is that thermal tides in the atmosphere can create a strong enough torque to balance the stellar tidal torque on the mantle, as is expected to be the case on Venus (Chapman and Lindzen 1970; Ingersoll and Dobrovolskis 1978; Correia and Laskar 2001; Leconte et al. 2015; Auclair-Desrotour et al. 2017). For this process to be efficient, the planet must be close enough from the star so that tides in general are able to affect the planetary spin, but far enough so that bodily tides are not strong enough to overpower atmospheric tides. In the TRAPPIST-1 system, this zone rests well beyond the position of the seven known planets (see Fig. 3 of Leconte et al. 2015). Atmospheric tides should thus be unable to significantly affect the spins of TRAPPIST-1 planets.

Another possibility for planets on an eccentric enough orbit is capture into a higher order spin-orbit resonance (Goldreich and Peale 1966), i.e. higher than the synchronous rotation. Using the formalism of Ribas et al. (2016), Turbet et al. (2018) calculated that the eccentricity of a given planet in the TRAPPIST-1 system must be above $\sim 10^{-2}$ to be possibly captured in a higher order spin-orbit resonance. Probability of capture becomes greater than $10 \%$ only for an eccentricity greater than 0.03 (compatible with the calculations of Makarov (2012), although made for a different system). Simulations of the system dynamics accounting for tides and planet-planet interactions (Turbet et al. 2018) seem to show that such eccentricities are on the very high end of the possible scenarios. This was also confirmed in the TTV analysis of Grimm et al. (2018) showing that all TRAPPIST-1 planets most likely have eccentricities equal or below $10^{-2}$. Recently, Auclair-Desrotour et al. (2019) developed a tidal model for ocean planets and showed that, although resonances of oceanic modes are likely to decrease the critical eccentricity for which eccentric planets can be trapped in 3:2 spin-orbit resonance, this should not directly affect the end spin-state of the TRAPPIST-1 planets because their eccentricities are likely too low.

In summary, none of the two aforementioned processes should be strong enough to counteract bodily tides so that all TRAPPIST-1 planets are mostly likely today in a synchronousrotation state.

However, it has been recently shown by Vinson et al. (2019) using a pendulum spin model introduced in Vinson and Hansen (2017) that due to planet mutual interactions, some of the TRAPPIST-1 planets may be pushed in a specific spin state of the synchronous rotation with (i) significant libration of the spin state and/or (ii) complete circulation of the spin state. In the numerical simulations of Vinson et al. (2019), the timescale for the spin libration and/or circulation is on the order of several Earth years, i.e. on the order of hundreds of orbits of TRAPPIST-1 planets. They also noted that these libration and/or circulation spin-states are quasi-stable and that TRAPPIST-1 planets can shift from one state to another on the order of $10^{3}-10^{5}$ years. The exact timescales depend on the planet considered, and tidal dissipation factors assumed.

Whether the planets are in a classic synchronous state, in a higher order spin-orbit resonance, or in a synchronous state with strong libration, possibly even circulation, would have profound implications for the possible climates and atmospheres of TRAPPIST-1 planets (see an example in Fig. 1 of Turbet et al. 2016). 
Last but not least, it is important to mention that tidal heating is likely the dominant source of internal heating at least for the innermost TRAPPIST-1 planets (Turbet et al. 2018; Barr et al. 2018; Makarov et al. 2018; Dobos et al. 2019). For instance, Turbet et al. (2018) calculated that the mean surface tidal heating flux on TRAPPIST-1b is within 0.7$40 \mathrm{~W} \mathrm{~m}^{-2}$, depending on the tidal dissipation factor assumed and eccentricity calculated. Similar orders of magnitude for the tidal heating flux were obtained by Papaloizou et al. $2018\left(<5 \mathrm{~W} \mathrm{~m}^{-2}\right.$ ), Barr et al. 2018 (between 0.8 and $4 \mathrm{~W} \mathrm{~m}^{-2}$ ), and Dobos et al. 2019 (between 0.1 and $2 \mathrm{~W} \mathrm{~m}^{-2}$ ). Differences are due to different tidal dissipation factors assumed and different eccentricities calculated and/or assumed. Interestingly, the viscosity of the mantle depends on its physical state (e.g. temperature), which itself depends on the rate of heating. Makarov et al. (2018) thus argued that in planets with strong potential heating (such as TRAPPIST-1b) the tidal dissipation might be determined by a feedback loop (between the physical state of the planetary interior and the heating rate) rather independently from the exact eccentricity.

All these tidal heat flux estimates are likely much larger for TRAPPIST-1b than expected radiogenic heating. On Earth, the typical radiogenic heating is $\sim 0.08 \mathrm{~W} \mathrm{~m}^{-2}$ (Davies and Davies 2010; Davies 2013) but it is likely to be lower on TRAPPIST-1 planets given the age of the system (Burgasser and Mamajek 2017), about twice that of the solar system. Uncertainties on the initial inventory of thermally important radioactive elements as well as on the stellar age are high (Burgasser and Mamajek 2017), which may affect this conclusion. Last, we note that the mechanism of electromagnetic induction heating proposed by Kislyakova et al. (2017) should have a negligible contribution to the surface heat flux. The maximum induction heating estimated for TRAPPIST-1 planets by Kislyakova et al. (2017) yields a surface flux of $2 \times 10^{-2} \mathrm{~W} \mathrm{~m}^{-2}$.

In summary, tidal heating is expected to be the dominant interior heating process for TRAPPIST-1 inner planets, but likely not for outer ones. The tidal heating flux may be high enough for innermost planets that it could melt the mantle and possibly trigger intense volcanic activity and thus outgassing of volcanic gases. However, and for all planets, tidal heating is at least two orders of magnitude lower than the stellar flux they received, indicating that the direct surface and atmospheric warming from tides is negligible.

\section{Constraints from Transit Observations}

Since the initial discovery of the TRAPPIST-1 system, many ground and space-based largeaperture telescopes have been used to measure transits of all the seven TRAPPIST-1 planets in a large range of wavelengths. Figure 5 summarizes all the transit observations that have been published as of December 2019 (de Wit et al. 2016; Gillon et al. 2017; Bourrier et al. 2017b,a; de Wit et al. 2018; Delrez et al. 2018; Ducrot et al. 2018; Luger et al. 2017b; Burdanov et al. 2019; Wakeford et al. 2019; Ducrot et al. 2020) as a function of the wavelength of observation, producing the most complete transmission spectra of TRAPPIST-1 planets to date. At least three effects can explain radius variations with wavelength: (i) atomic/molecular absorptions by TRAPPIST-1 planetary atmospheres, (ii) instrumental biases and (iii) contamination by the stellar activity (e.g. presence of spots). Below, we review the information gathered and lessons learned from these multi-wavelengths transit observations. 

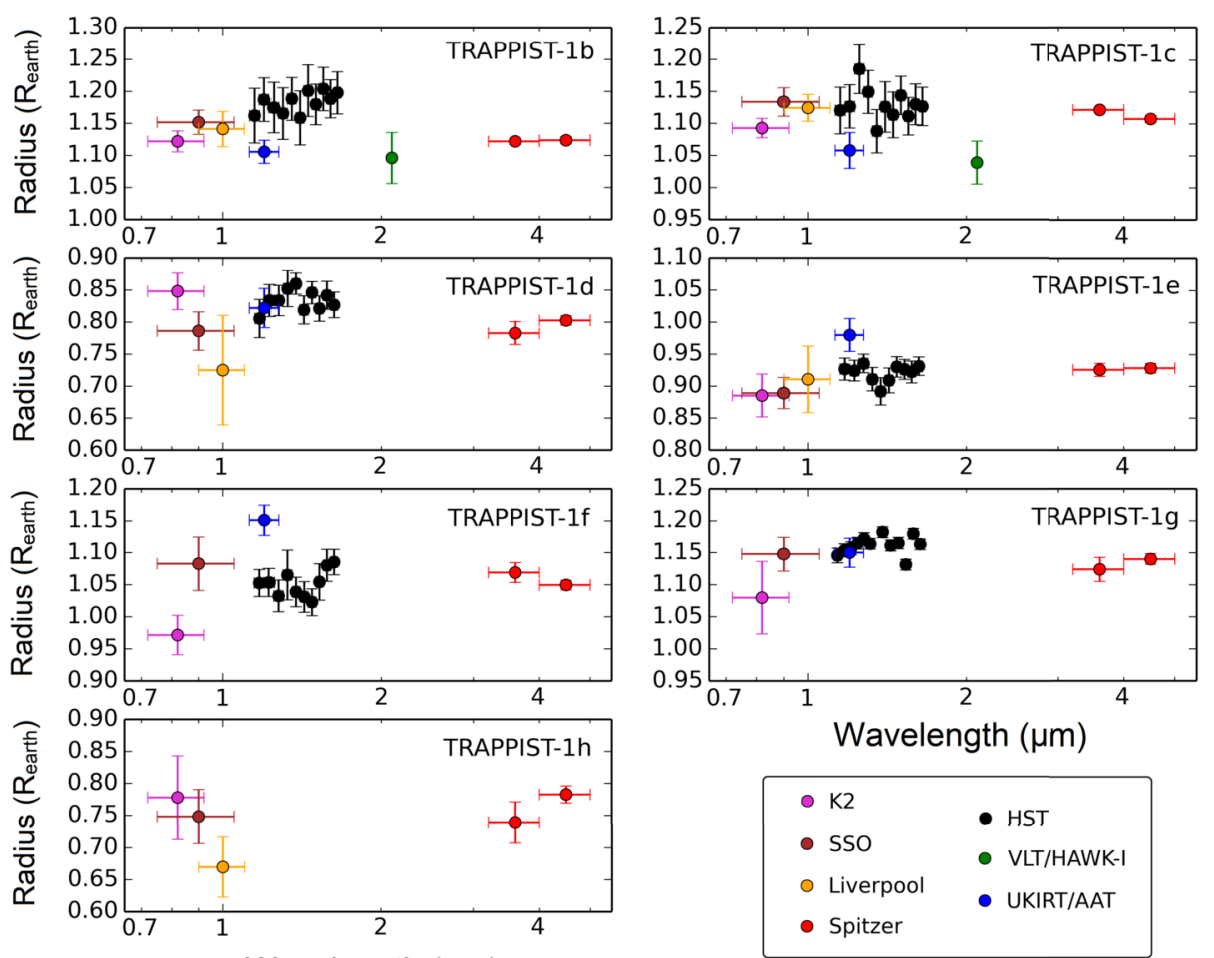

Fig. 5 This figure shows transit spectra (in Earth radius units) of the seven TRAPPIST-1 planets. These spectra were constructed using the transit depth measurements obtained with Spitzer (Gillon et al. 2017; Delrez et al. 2018; Ducrot et al. 2018, 2020), HST (de Wit et al. 2016, 2018; Wakeford et al. 2019), K2 (Luger et al. 2017b; Ducrot et al. 2018), SPECULOOS-South Observatory aka SSO and Liverpool telescope (Ducrot et al. 2018), VLT/HAWK-I, UKIRT and AAT (Burdanov et al. 2019). These transit depths were then converted into transit radii using the TRAPPIST-1 stellar radius estimate of Van Grootel et al. (2018), i.e. $R_{\star}=0.121 R_{\odot} \pm 0.003$. The absolute error bars on the planetary radii due to the uncertainty on the radius of the star (about 2.5\% according to Van Grootel et al. 2018) have not been applied here, because it is the relative uncertainties that are of interest here. Note that some of the transit observations (e.g. ground-based observations, HST/WFC3 observations) may not have a reliable absolute monochromatic baseline level (Ducrot et al. 2018, 2020)

\subsection{No Cloud-Free Hydrogen-Dominated Atmospheres on Most TRAPPIST-1 Planets}

Hubble Space Telescope (HST) observations of the transits of TRAPPIST-1 planets (de Wit et al. 2016, 2018) have brought the strongest constraint so far on the possible atmospheres of TRAPPIST-1 planets. Transits were observed on HST using the WFC3/IR instrument (1.1$1.7 \mu \mathrm{m}$ ) first on TRAPPIST-1bc (de Wit et al. 2016) and then on TRAPPIST-1defg (de Wit et al. 2018; Wakeford et al. 2019). Improvements in the data reduction pipeline of the HST transit observations were proposed later in Zhang et al. (2018), which reported a net increase in the efficiency of HST observations by $\sim 25 \%$.

de Wit et al. (2016) and de Wit et al. (2018) produced synthetic spectra of $\mathrm{H}_{2}$-dominated cloud-free atmospheres and compared them with real HST data (see black data points in Fig. 5). They showed that the lack of prominent features in the HST spectra rules out cloud- 
free (and haze-free) hydrogen-dominated atmospheres for TRAPPIST-1b, c, d, e, f at 12, $10,8,6$, and $4 \sigma$, respectively. For example, de Wit et al. (2016) showed that the expected amplitude of the $1.4 \mu \mathrm{m}$ water feature in a hydrogen-dominated, low molecular weight atmosphere is $\sim 2000 \mathrm{ppm}$ (in transit depth) for TRAPPIST-1b and c, corresponding to planetary radius variation $\sim 0.15-0.20 R_{\oplus}$ which are not seen in HST transit observations. de Wit et al. (2018) and Moran et al. (2018) calculated that the amplitude of the same feature is less than $1000 \mathrm{ppm}$ (or less than $0.07 R_{\oplus}$ ) for TRAPPIST-1g, mostly because the atmosphere is colder, thus reducing the atmospheric scale height $H=\frac{R T}{M g}$, where $R$ is the perfect gas constant, $T$ the atmospheric temperature, $M$ the mean molar mass of the atmosphere, and $g$ the gravity. As a result, a clear hydrogen-rich atmosphere cannot be firmly ruled out for TRAPPIST-1g with HST observations only (de Wit et al. 2018; Moran et al. 2018).

Moran et al. (2018) then performed atmospheric calculations to explore if more sophisticated models of hydrogen-rich atmospheres (including higher metallicity, clouds, photochemical hazes) could also be ruled out by HST observations. They determined that $\mathrm{H}_{2}$-rich atmospheres (with solar metallicity ${ }^{4}$ ) with high altitude clouds (at pressures of 12 mbar or lower) are consistent with the HST observations for TRAPPIST-1d and e. Moreover, they found that HST observations cannot rule out (at $3 \sigma$ ) hydrogen-dominated atmosphere (with a cloud layer at 0.1 bar) with 300,100 and $60 \times$ solar metallicity for TRAPPIST-1d, e and f, respectively. This stems from the fact that high metallicity hydrogen-dominated atmosphere have a much larger mean molecular weight, and thus a lower atmospheric scale height and therefore reduced atmospheric feature amplitudes.

In conclusion, most of TRAPPIST-1 planets are unlikely to have an extended hydrogendominated atmosphere. However, this possibility cannot be completely ruled out by the HST/WFC3 observations, because either (i) a very high altitude cloud cover or (ii) very high metallicity $\mathrm{H}_{2}$-dominated atmospheres could in principle both fit HST/WFC3 observations. Furthermore, Lyman- $\alpha$ observations obtained with HST/STIS showed marginal flux decrease at the time of TRAPPIST-1 $b$ and $c$ transits, which could hint at the presence of extended hydrogen exospheres around these planets Bourrier et al. (2017b,a). More HST/STIS observations of TRAPPIST-1 planetary transits (HST Proposal ID 15304, PI: Julien de Wit) are expected to put more precise constraints in a near-future.

\subsection{Possible Indications of Stellar Contamination}

No significant absorption features have been detected so far in any individual transmission spectra (see Fig. 5). Yet, it is possible to increase the SNR of transit observations by combining the transmission spectra of all planets (see black dots in Fig. 6). Part of the variations seen in the combined transit spectrum may be due to the presence of atmospheric absorptions, but also likely to the presence of heterogeneities in the photosphere of the star TRAPPIST- 1. This includes the presence of both occulted and unocculted (cold) spots and/or (hot) faculae.

When a planet transits in front of its host star, the transit depth of the planet is measured by taking the difference of the disk-integrated stellar spectrum between in and out of transit. It is usually assumed in this calculation that the disk-integrated spectrum is identical to the light incident on the planet and its atmosphere. In reality, however, the planet is occulting only a small region within the transit chord, and only at a given time. Due to the presence of spots, faculae, and even latitudinal temperature gradients, the spectrum of this small region

\footnotetext{
${ }^{4}$ Metallicity refers to the overall heavy-element abundance. An atmosphere with solar metallicity therefore has the same composition of heavy elements (in particular carbon and oxygen, which can be converted into water vapour and methane) as the Sun.
} 
may differ significantly from the disk-averaged spectrum of the star. As a result, the presence of heterogeneities in the stellar photosphere can bias transit observations.

Rackham et al. (2018) recently called into question the fidelity of the transit observationsmore specifically of the HST measurements (de Wit et al. 2016, 2018)—of TRAPPIST-1 planets, because of possible contamination of the transmission spectra by the presence of spots and faculae in the photosphere of the star TRAPPIST-1. This contamination is also known as the 'transit light source effect'. For this, Rackham et al. 2018 (see their equations (1)-(3)) developed a simple stellar contamination spectrum model based on three components (photosphere, unocculted spot, unocculted faculae) each with a given temperature and spatial coverage. Each component can be fitted with a combination of three synthetic stellar spectra (e.g. PHOENIX spectra) at three different temperatures. The original model of Rackham et al. (2018) assumes that no heterogeneities (e.g. spots or faculae) are present within the transit chord; or, if they are, that they can be identified in the light curve and properly taken into account. However, because the precision of observations may not allow stellar surface heterogeneities within the transit chord to be reliably detected, Zhang et al. (2018) (final published version; see their equation (7)) proposed an extension to the model of Rackham et al. (2018) by taking into account the presence of spots and faculae in the transit chord. They also allowed the covering fraction of spots and faculae in the transit chord to differ from the whole-disk values. Based on the formalism of Rackham et al. (2018), two stellar contamination scenarios have been proposed so far:

1. The first model (Rackham et al. 2018; Zhang et al. 2018) assumes a typical spot size of thousands of $\mathrm{km}$, or $\sim 0.04 \%$ of the projected stellar disk of TRAPPIST-1 (i.e. very similar in size to large spot groups on the Sun's photosphere). This assumption is based on the observation that long-baseline monitoring of TRAPPIST-1 using the Spitzer Space Telescope (Gillon et al. 2017; Delrez et al. 2018; Ducrot et al. 2020) and HST/WFC3 (de Wit et al. 2016) shows no definitive evidence of spot crossing events; and that the spot size must be significantly lower than $\sim 0.5 \%$ of the projected stellar disk otherwise it should have impacted the stellar variability of TRAPPIST-1 in HST/WFC3 and Spitzer/IRAC observations (Rackham et al. 2018). Using this assumption, the stellar contamination model is based on three components (i.e. the photosphere is decomposed into three regions of three different sizes and temperatures) designed to fit existing transit data from K2, SPECULOOS-South, HST/WFC3 and Spitzer/IRAC channel 2 (4-5 $\mu \mathrm{m})$, as illustrated by the blue curve in Fig. 6. Their complete transmission spectrum (blue line in Fig. 6) is fully consistent with stellar contamination, except with the more recent Spitzer/IRAC channel $1(3.15-3.9 \mu \mathrm{m})$ presented in Ducrot et al. (2020). The three components of this model (Zhang et al. 2018) are (i) a photosphere ( $T=2400 \mathrm{~K})$, (ii) hot faculae $(T=3000 \mathrm{~K})$ covering $50 \%$ of the projected stellar disk and cold spots ( $T=2000 \mathrm{~K}$ ) covering $40 \%$ of it. Note that in the final model of Zhang et al. 2018 (published version, and not the first submitted arXiv version ${ }^{5}$ ), the spot coverage is much

\footnotetext{
${ }^{5}$ Zhang et al. 2018 (first arXiv version) initially used the original formalism of Rackham et al. 2018 (see equation (3)) to build their stellar contamination spectrum, i.e. assuming no heterogeneities - spots or faculae-are present within the transit chord. Ducrot et al. (2018) compared the model of Zhang et al. 2018 (first arXiv version) and discarded it (by more than 10 $\sigma$ ) mostly using K2 observations in the visible wavelengths. Meanwhile, Zhang et al. 2018 (published version) updated their model to include the presence of spots and faculae in the transit chord, considering the precision of observations may not allow stellar surface heterogeneities within the transit chord to be reliably detected. In their revised model (blue line in Fig. 6; see also equation (7) of Zhang et al. 2018), they assume the transit chord is composed of the same three spectral components as the integrated disk, but they allow their covering fractions to differ from the whole-disk values.
} 
COMBINED TRANSMISSION SPECTRUM OF TRAPPIST $-1 b+c+d+e+f+g$ PLANETS

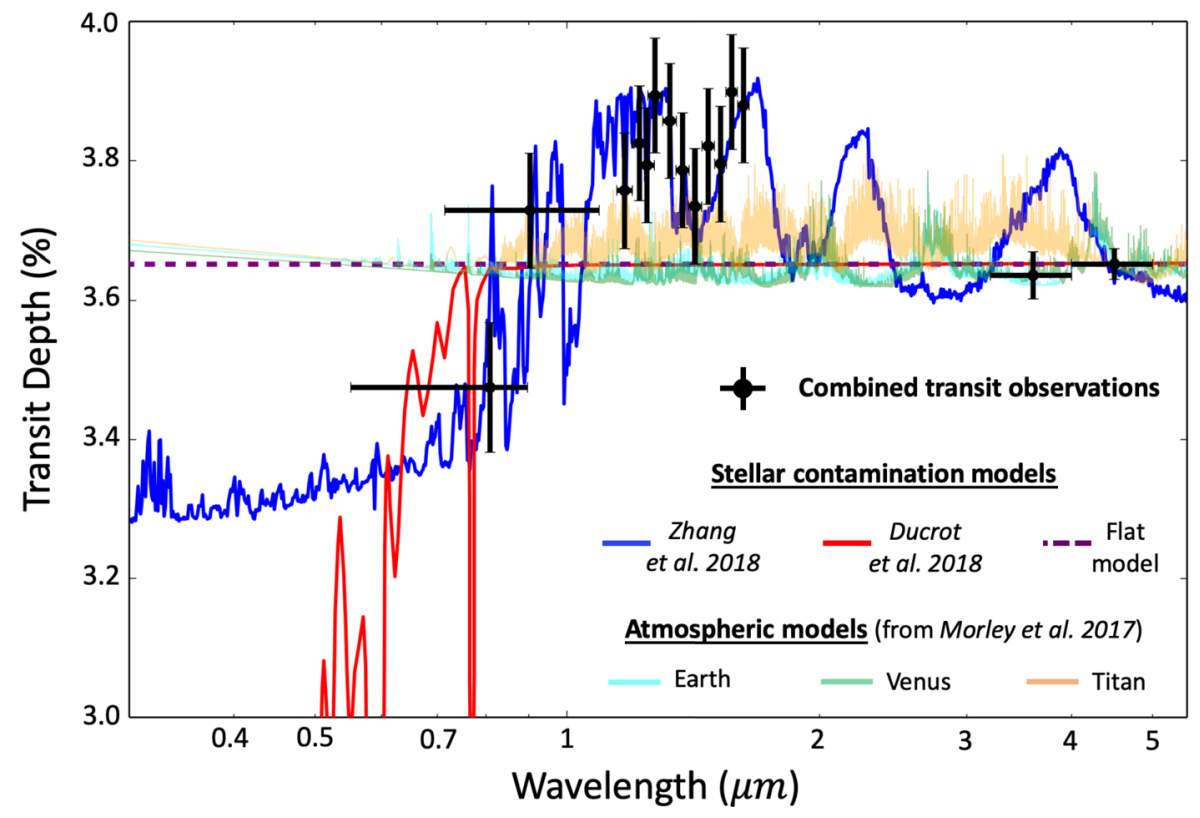

Fig. 6 Stellar contamination models fit to the K2+SPECULOOS-South+HST/WFC3+Spitzer/IRAC channels 1 and 2 (de Wit et al. 2016; Gillon et al. 2017; Luger et al. 2017b; Delrez et al. 2018; de Wit et al. 2018; Ducrot et al. 2018, 2020) combined TRAPPIST-1 transmission spectra for planets $\mathrm{b}+\mathrm{c}+\mathrm{d}+\mathrm{e}+\mathrm{f}+\mathrm{g}$ (black points and error bars, in transit depth \% units). The blue stellar contamination spectrum (Zhang et al. 2018) corresponds to a three components model with (i) a photosphere $(T=2400 \mathrm{~K})$, (ii) hot faculae $(T=3000 \mathrm{~K})$ covering $50 \%$ of the projected stellar disk and cold spots $(T=2000 \mathrm{~K})$ covering $40 \%$ of it. The red stellar contamination spectrum (Morris et al. 2018a; Ducrot et al. 2018) corresponds to a two-component model with (i) a photosphere $(T=2500 \mathrm{~K})$ and (ii) a few very bright spots ( $T=5300 \mathrm{~K})$. The dashed purple stellar contamination spectrum corresponds to a flat model (i.e. no stellar contamination), which also corresponds to the best fit scenario in Wakeford et al. (2019). For each contamination spectrum, a small offset was added to ensure that each spectrum is compatible with the Spitzer/IRAC $4.5 \mu \mathrm{m}$ transit measurement. Note that in the three contamination models (blue, red, and dashed purple lines), the signal (when fitted) is assumed to be fully stellar, i.e. no contribution from wavelength-dependent absorption by planetary atmospheres. The pale (i) cyan, (ii) green and (iii) orange lines correspond to combined synthetic transmission spectra from planetary atmospheres all made of (i) Earth-like, (ii) Venus-like and (iii) Titan-like compositions. These combined spectra were computed by summing the synthetic spectra of TRAPPIST- $1 \mathrm{~b}+\mathrm{c}+\mathrm{d}+\mathrm{e}+\mathrm{f}+\mathrm{g}$ from Morley et al. (2017). They assume no stellar contamination

lower in the transit chord that in the rest of the planet suggesting, (i) a latitudinal variation of spot coverage on the star, and (ii) that it is important to take this possibility into account in the fit.

2. The second stellar contamination model (Morris et al. 2018a; Ducrot et al. 2018) is based on the observation that the activity of the star TRAPPIST-1 as measured with K2 and Spitzer (Morris et al. 2018a) was best described by a two-component model with (i) a photosphere $(T=2500 \mathrm{~K})$ and (ii) a few very bright spots $(T=5300 \mathrm{~K})$ with a fractional lower limit coverage of $\sim 0.005 \%$. In fact, Morris et al. (2018a) argued that cold spots (if present) should produce variability in the Spitzer light curves that is yet absent in existing data, motivating therefore a two-component model without cold spots. Additionally, Ducrot et al. (2018) reported that the photosphere of TRAPPIST-1 is most likely 
described by a base photosphere with a small fraction of hot faculae $(T>4000 \mathrm{~K})$. This stems from the fact that their stellar contamination model (red line in Fig. 6) is fully consistent with existing transit observations, including the recent Spitzer/IRAC channel 1 (3.15-3.9 $\mu \mathrm{m})$ data presented in Ducrot et al. (2020), but not the HST $(1.1-1.7 \mu \mathrm{m})$ data (de Wit et al. 2016, 2018) which they discarded from their analysis (see discussion hereafter). Ducrot et al. (2018) noted that the stellar photosphere may in principle also be fitted with high latitude cold spots.

Both models appear to be roughly consistent with stellar contamination in the combined transmission spectrum. However, depending on which model is correct, our ability to characterize in detail the possible atmospheres of TRAPPIST-1 planets with forthcoming large aperture telescopes such as JWST may be strongly affected. This is particularly critical in infrared wavelengths for which the two models have very different predictions. While the model of Morris et al. (2018a) and Ducrot et al. (2018) predicts a nearly flat contamination spectrum for wavelengths $>0.7 \mu \mathrm{m}$ (red line in Fig. 6), the model of Rackham et al. (2018) and Zhang et al. (2018) predicts strong spectral variations (blue line in Fig. 6). The latter stellar contamination spectrum would alter the transit depths of the TRAPPIST-1 planets for planetary atmospheric species by roughly $1-15 x$ the strength of planetary features, for wavelengths $>0.7 \mu \mathrm{m}$, thus significantly complicating JWST follow-up transit observations of this system. Moreover, this contamination would also introduce a bias as high as $\sim 2.5 \%$ (more likely an overestimate) on planetary radii estimated with Spitzer IRAC channel 2 (Rackham et al. 2018), and could thus bias the density estimates.

Below, we review the pros and cons of the two models:

1. Fit of the combined transmission spectrum: Both models provide a reasonable fit of existing transit data (see red and blue lines in Fig. 6). However, if HST data is included in the fit, the model of Zhang et al. (2018) performs very well and the model of Ducrot et al. (2018) can be discarded. Without HST data, the model of Ducrot et al. (2018) performs better than the model of Zhang et al. (2018).

2. HST data and the inverted $1.4 \mu \mathrm{m}$ water vapour feature: Ducrot et al. (2018) reported that the HST observations (de Wit et al. 2016, 2018) may not have a reliable absolute monochromatic baseline level, due to orbit-dependent systematic effects. This is a good argument to remove the HST monochromatic data from the global fit of the stellar contamination model. However, the chromatic variation of transit depth within the 1.1$1.7 \mu \mathrm{m}$ range can in principle be used separately as an additional constraint. While de Wit et al. $(2016,2018)$ do not see any strong evidence for variations within the HST/WFC3 band, Zhang et al. (2018) show-by improving on the HST/WFC3 data analysis pipeline and summing the contributions of all planets from $b$ to $g$ - the presence of a strong inverted water vapour feature (black data points between 1.1 and $1.7 \mu \mathrm{m}$, in Fig. 6) in the six-planets combined transmission spectrum. Zhang et al. 2018 (see their Fig. 11) also found that the same water vapour inverted feature is present in all possible five-planet combined transmission spectra, indicating it is not solely due to the spectrum of an individual planet. The presence of this water vapour inverted feature-if confirmed-is a strong evidence for the reliability of the stellar contamination model of Zhang et al. (2018).

3. Planetary radius bias: The model of Rackham et al. (2018) and Zhang et al. (2018) predicts a $\sim 2.5 \%$ radius bias in Spitzer/IRAC infrared wavelengths, while the model of Morris et al. (2018a) and Ducrot et al. (2018) does not predict any. Morris et al. (2018c) re-evaluated the TRAPPIST-1 planets transit depths by estimating - in the Spitzer transit light curves-(i) the durations of the ingress/egress, (ii) the duration of the transit and 
(iii) the impact parameter. The method is detailed in Morris et al. (2018b) (see equations (5)-(8)). This method, also known as the transit light-curve 'self-contamination' technique, is very weakly affected by the presence of stellar heterogeneities and can thus be used in principle to derive transit depth estimates that are not biased by the presence of heterogeneities in the stellar photosphere (Morris et al. 2018b). By using this method, Morris et al. (2018c) found consistent transit depth measurements between the traditional method and the light curve self-contamination method of Morris et al. (2018b) and concluded on the absence of statistically significant evidence of stellar contamination in the Spitzer infrared wavelengths, thus supporting the model of Morris et al. (2018a) and Ducrot et al. (2018). However, Zhang et al. (2018) reported that the light curve selfcontamination method has such large uncertainties (see Fig. 3 of Morris et al. 2018c) that they do not have the sensitivity to probe the level of stellar contamination predicted by the model of Rackham et al. (2018) and Zhang et al. (2018).

4. K2/I band/Spitzer stellar variability and size of stellar heterogeneities: While the Rackham et al. (2018) and Zhang et al. (2018) model assumed that the heterogeneities (two components) in TRAPPIST-1 photosphere are similar in size to those in the Sun's photosphere, Morris et al. (2018a) and Ducrot et al. (2018) model assumed instead an empirically driven hypothetical spot distribution for TRAPPIST-1, consisting of (one component) a few small, bright (hot) spots. The former model is based on the assumption that the $\sim 1 \%$ TRAPPIST-1 typical I-band variability (Rackham et al. 2018) indicates the absence of large/giant heterogeneities in TRAPPIST-1 photosphere. The latter model is based instead on the assumption that the variability in the K2 and Spitzer light curves is driven by rotational modulation due to starspots (Morris et al. 2018a). While K2 (400$900 \mathrm{~nm}$ ) light curves show a variability of $\sim 1.25 \%$ with a period of 3.3 days (Luger et al. 2017b) comparable to the typical I-band variability (Rackham et al. 2018), Spitzer (4$5 \mu \mathrm{m}$ IRAC channel) light curves show very little variability $\leq 0.1 \%$ (Delrez et al. 2018; Morris et al. 2018a; Ducrot et al. 2020). This observation led Morris et al. (2018a) to conclude that large $\left(\sim 10^{4} \mathrm{~km}\right)$ dark spots should be absent in the stellar photosphere, otherwise they would produce much stronger variability in Spitzer light curves (see Fig. 2 in Morris et al. 2018a and discussion in Ducrot et al. 2018). A two-component photosphere model (Morris et al. 2018a) with a few very bright spots can however drive the modulation with a 3.3 day period in the $\mathrm{K} 2$ bandpass without generating a corresponding signal in the Spitzer $4.5 \mu \mathrm{m}$ band, in agreement with the observations. Note that these observations do not discard the model of Rackham et al. (2018) and Zhang et al. (2018) which assumed much smaller dark spots (a factor of 10 lower than in Morris et al. (2018a)). This also means that the model of Morris et al. (2018a) and Ducrot et al. (2018) could be improved by adding a third component of small, dark spots. Meanwhile, Wakeford et al. (2019) noted that the spot temperature posterior distribution of Zhang et al. 2018 (see their Fig. 13 and 20) is truncated to high temperature, potentially preventing it from reaching the parameter space of very hot bright spots found in Morris et al. (2018a) and Ducrot et al. (2018).

5. No clear spot crossing events during transits: Analyses of observations in the visible and near-IR carried out by K2, SPECULOOS and the Liverpool telescope do not show transit depth variability arising from stellar spot crossing events during transits (Delrez et al. 2018; Ducrot et al. 2018, 2020). Ducrot et al. (2020) identified a few outliers in the Spitzer transit light curves but they interpreted most of them as instrumental noise. This means that spots must be either cool and smaller than the size of the planets (which would reduce the probability and amplitude of a spot occultation) or reside outside of the transit chords (Morris et al. 2018a). These observations are particularly constraining 
on the bright spots, that should be absent of the transit chords of TRAPPIST-1. This is surprising, given the transit chords represents $\sim 56 \%$ of an hemisphere (Delrez et al. 2018).

To conclude, it is still unclear which of the two models is the best predictor of the stellar contamination. In the model of Rackham et al. (2018) and Zhang et al. (2018), the stellar contamination is so strong in the infrared (by comparison to the expected amplitude of the atmospheric molecular absorption features; see Fig. 6) that it is undoubtedly the most important limitation to our ability to characterize the atmosphere of the TRAPPIST-1 planets with the James Webb Space Telescope. In the model of Morris et al. (2018a) and Ducrot et al. (2018), the stellar contamination is so flat in the infrared wavelengths that observations made with the two channels of Spitzer/IRAC could already be used to discard some high mean molecular weight atmospheres. For instance, if the stellar contaminaton model of Morris et al. (2018a) and Ducrot et al. (2018) is correct, then we conclude based on Fig. 6 (orange line, for Titan-like atmospheres) that all TRAPPIST-1 planets cannot be $\mathrm{CH}_{4}$ dominated (Ducrot et al. 2020). This stems from the fact that the $3.3 \mu \mathrm{m} \mathrm{CH}_{4}$ band should otherwise produce a significant variation of transit depth between the two Spitzer/IRAC warm channels that is not observed (Ducrot et al. 2018, 2020).

With a few exceptions (fit of HST data, Spitzer IRAC channel 1 data), both models appear to be so far broadly compatible with observational data. Besides, and as supported by the recent work of Wakeford et al. (2019), it seems that the two models could be reconciled by (i) adding a small, dark spot component and (ii) allowing for a very hot bright spot component. Better constraints on the nature of stellar heterogeneities present in the photosphere of TRAPPIST-1 is key to characterizing TRAPPIST-1 planetary atmospheres.

\section{Constraints from Numerical Modelling}

In this section, we review the various theoretical and numerical efforts that have been recently undertaken to better constrain the nature of the possible atmospheres of TRAPPIST-1 planets. Below, we list all common gases in known planetary atmospheres $\left(\mathrm{H}_{2}, \mathrm{H}_{2} \mathrm{O}, \mathrm{CO}_{2}\right.$, $\mathrm{CH}_{4}, \mathrm{~N}_{2}, \mathrm{O}_{2}$ ) and discuss whether or not these gases may have predominantly accumulated in the atmospheres of TRAPPIST-1 planets, based on the results of numerical 1D and 3D climate models, photochemical models, and atmospheric escape models.

\section{$5.1 \mathrm{H}_{2} / \mathrm{He}$ Envelopes}

It has been argued (de Wit et al. 2016, 2018; Wakeford et al. 2019) based on HST transit observations that most of TRAPPIST-1 planets are unlikely to be endowed with a hydrogendominated atmosphere, unless high altitude clouds/hazes are present or high metallicity atmospheres are considered (Moran et al. 2018). This is discussed in more details in Sect. 4.1.

Here we argue that hydrogen-dominated atmospheres are unlikely for TRAPPIST-1 planets, based also on their mass and radius estimates. Using the numerical atmospheric calculations of $\mathrm{H}_{2}$-dominated atmospheres presented in Grimm et al. (2018), we constructed (see Fig. 7) mass-radius relationships for hydrogen-dominated atmospheres. For this, we assumed (i) a terrestrial core composition (Zeng et al. 2016), ${ }^{6}$ (ii) hydrogen envelopes of various mass fraction (from $10^{-6}$ to $10^{-2}$ ), (iii) atmospheric temperature-pressure profiles

\footnotetext{
${ }^{6}$ Note that here and in the following subsection, we adopt a terrestrial core composition to construct massradius relationships. Although the core composition is not known (and is thus a highly degenerate quantity),
} 


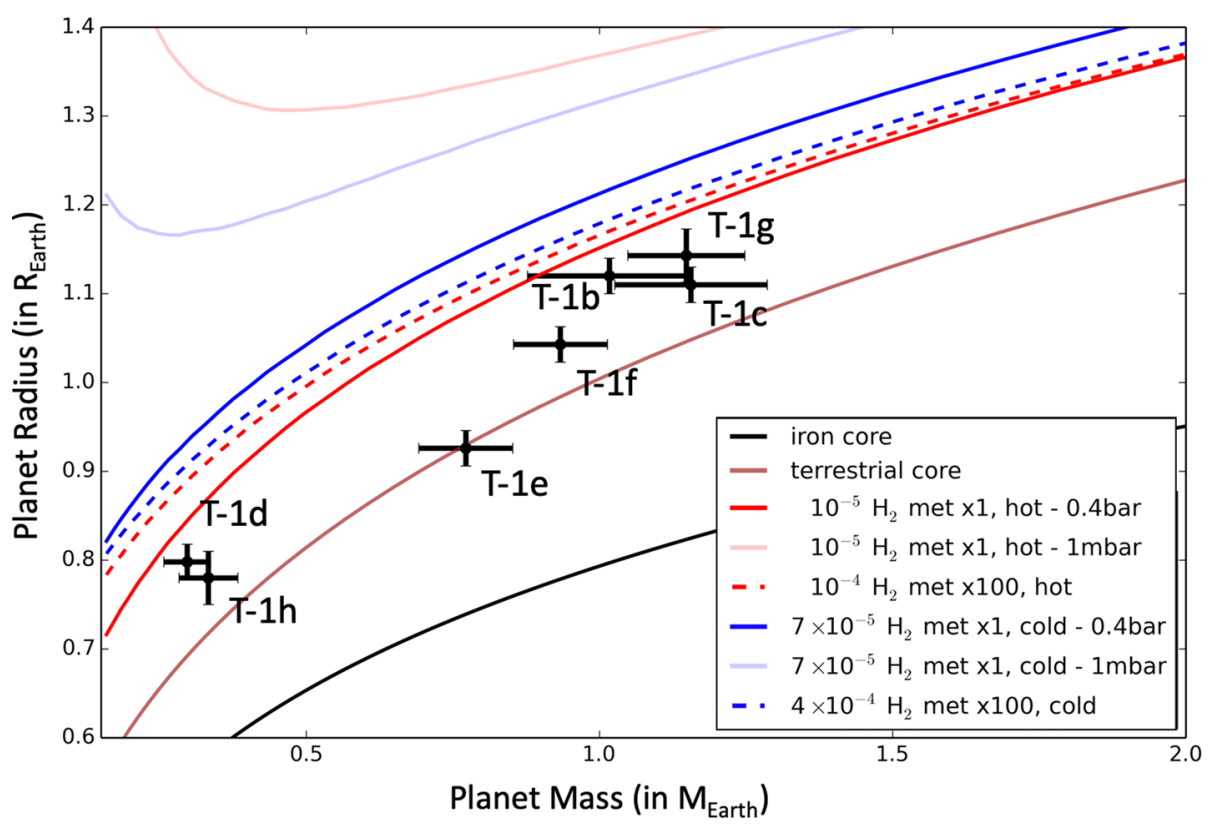

Fig. 7 Mass-radius relationships for various interior compositions and hydrogen envelope masses. The mass-radius relationships for planets endowed with hydrogen envelopes were constructed (i) assuming a core of terrestrial composition (Zeng et al. 2016), (ii) endowed with a hydrogen envelope of 1x solar metallicity (solid lines) or 100x solar metallicity (dotted lines) for $\mathrm{H}_{2} \mathrm{O}$ and $\mathrm{CH}_{4}$. Red lines (and blue lines, respectively) indicate a scenario where the atmospheric temperature profile has been calculated in the irradiation condition of TRAPPIST-1b (of TRAPPIST-1h, respectively), hence the name 'hot' (and 'cold', respectively). All transit radii were computed assuming a transit pressure of 0.4 bar, which is a conservative assumption based on the results of Grimm et al. (2018) (Table 4). We also plotted the expected transit radii assuming a transit pressure at $1 \mathrm{mbar}$ (pale red and blue lines). For comparison, we added the masses and radii of the seven TRAPPIST-1 planets measured from Grimm et al. (2018) and Ducrot et al. (2020), with their associated $1 \sigma$ error bars. For reference, we also added a terrestrial composition (Zeng et al. 2016) that ressembles that of the Earth, and a pure iron core composition (Seager et al. 2007)

based on the atmospheric numerical climate calculations of Grimm et al. (2018). For simplicity, we calculated mass-radius relationships based on two distinct atmospheric scenarios: first, a 'hot' scenario using the temperature-pressure profile calculated for a planet receiving the irradiation of TRAPPIST-1b, assuming 1x solar metallicity; and a 'cold' scenario, using the temperature-pressure profile calculated for a planet receiving the irradiation of TRAPPIST-1h, assuming 1x solar metallicity. At first order, numerical atmospheric models show (Grimm et al. 2018) that these temperature profiles can be described by an isothermal stratosphere (down to $\sim 0.1$ bar) complemented with a dry convective troposphere (from $\sim 0.1$ bar down to the surface). The transit radius of the planet was then computed by integrating the hydrostatic equation (assuming a gravity varying with altitude) using the Saumon

it has been shown by Unterborn et al. (2018) — using the Hypatia catalog (Hinkel et al. 2014) - that F-G-K stars of similar metallicity to TRAPPIST-1 have a Fe/Mg $=1.72 \pm 0.46$ in mass ratios, which gives a Fe/Si ratio of $1.49 \pm 0.4$ (while fixing the $\mathrm{Mg} / \mathrm{Si}$ mass ratio to 0.87 ). These values are similar to the solar mass ratio estimates of $\mathrm{Fe} / \mathrm{Si}=1.69$ and $\mathrm{Mg} / \mathrm{Si}=0.89$ (Lodders et al. 2009), which motivates the use of a terrestrial core composition in the construction of the mass-radius relationships. 
et al. (1995) equation of state and assuming a transit pressure of 0.4 bar, the latter being a conservative estimate for all planets according to the results of Grimm et al. 2018 (Table 4).

In the most conservative scenario (high metallicity, cold atmosphere, transit pressure at 0.4 bar meaning the atmosphere is assumed to be cloud-free), Fig. 7 shows that the maximum hydrogen-to-core mass fraction allowed (at $1 \sigma$ ) by measured radii and masses of TRAPPIST-1 planets (Grimm et al. 2018; Ducrot et al. 2020) is roughly $4 \times 10^{-4}$. If the atmospheres were to be cloudy, then this maximum hydrogen content would be further reduced (e.g. down to $\sim 10^{-4}$ for a cloud top at 1 mbar).

According to Wheatley et al. (2017), TRAPPIST-1 planets receive between $3 \times 10^{3}$ (for planet b) and $10^{2} \mathrm{erg} \mathrm{s}^{-1} \mathrm{~cm}^{-2}$ (for planet h) XUV flux from the star TRAPPIST-1 today. This results in mass loss rates between $5 \times 10^{8}$ and $3 \times 10^{7} \mathrm{~g} \mathrm{~s}^{-1}$ for TRAPPIST-1b and $\mathrm{h}$, respectively, calculated in the energy-limited escape formalism (Bolmont et al. 2017a; Bourrier et al. 2017a), using revised mass and radius estimates of Grimm et al. (2018) and Ducrot et al. (2020). Furthermore, using the XUV flux history reconstruction of Bolmont et al. (2017a) and Bourrier et al. (2017a), we evaluate a cumulative (over 8 billion years) total hydrogen mass loss of $10^{23} \mathrm{~kg}$ (i.e. $2 \times 10^{-2}$ total mass fraction) and $10^{22} \mathrm{~kg}$ (i.e. $5 \times 10^{-3}$ total mass fraction) for TRAPPIST- $1 \mathrm{~b}$ and $\mathrm{h}$, respectively. This is 2000 and $100 \times$, respectively, the amount of hydrogen required to fit the mass and radius of TRAPPIST- $1 \mathrm{~b}$ and $\mathrm{h}$, assuming planets with a terrestrial core endowed with a high metallicity $\mathrm{H}_{2}$-dominated envelope. As a result, the hydrogen-to-core mass fraction envelope required to fit the mass-radius relationships would be removed in $\sim 100$ million years or less only, which is significantly lower than the estimated age of the TRAPPIST-1 system (Burgasser and Mamajek 2017).

This escape rate should be even larger considering that a $\mathrm{H}_{2}$-dominated atmosphere around a terrestrial-mass planet should be strongly extended (due to the reduction of gravity with altitude), and that the escape rate is expected to scale with $R_{\mathrm{p}}^{3}$ (Erkaev et al. 2007). This atmospheric expansion is illustrated in the $\mathrm{H}_{2}$-rich mass-radius relationships (see Fig. 7) that shows the difference of optical radius whether the atmosphere is assumed to be opaque at 0.4 bar (solid blue and red lines) i.e. cloud-free, or it is assumed to be opaque at $1 \mathrm{mbar}$ (pale solid blue and red lines) i.e. with high altitude clouds. Assuming the XUV radius (i.e. the planetary radius at which the atmosphere becomes optically thick to XUV photons) occurs at a pressure of $\sim 1$ nanobar (Murray-Clay et al. 2009; Lopez et al. 2012), we evaluate (using equation (16) of Grimm et al. 2018) that the XUV radius of TRAPPIST-1h is $\sim 1.5 \times$ that of its optical radius (for $100 \times$ solar metallicity), increasing the escape rate by a factor of $\sim 3$. For TRAPPIST- $1 b$ (and assuming $1 \times$ solar metallicity), the XUV radius can increase by $\sim 1.9$ thus increasing the escape rate by a factor of $\sim 7$.

In summary, based on $\mathrm{H}_{2}$-dominated mass-radius relationships for TRAPPIST-1 planets (Fig. 7), as well as hydrodynamical escape rate estimates (Owen and Mohanty 2016; Wheatley et al. 2017; Bolmont et al. 2017a; Bourrier et al. 2017a), $\mathrm{H}_{2}$-dominated envelopes are unlikely to be stable around any of the TRAPPIST-1 planets. Sustaining a hydrogen-rich atmosphere today is not theoretically impossible though, but it requires to consider a finetuned scenario where the planets were to be observed now exactly at the critical moment just before the complete loss of their initial hydrogen envelope (see Owen and Mohanty 2016, Fig. 13, for an example of hydrogen envelope evolution). However, it would not be possible to do this fine tuning for multiple planets in the same system. Besides, the absence of a large spread among TRAPPIST-1 planet densities is another argument against the presence of $\mathrm{H}_{2}$-dominated atmospheres. This stems from the fact that any significant change of $\mathrm{H}_{2}$ content-e.g. arising from variations in the hydrogen-rich gas accretion rates during the planet formation phase (Hori and Ogihara 2020) or from variations in the $\mathrm{H}_{2}$ escape rates (Bolmont et al. 2017b; Bourrier et al. 2017a) —among TRAPPIST-1 planets should produce a very different density, which is not observed (see Fig. 7). 
This argument, plus the HST/WFC3-IR transit measurements, are strong arguments against the presence of $\mathrm{H}_{2}$-dominated envelopes around TRAPPIST-1 planets. This is also supported by recent calculations (Hori and Ogihara 2020) showing the total mass loss of hydrogen-rich envelopes around TRAPPIST-1 planets is anyway likely higher than the amount of hydrogen-rich gas they can accrete during their formation.

\subsection{Water Envelope}

Based on the masses and radii measurements of Grimm et al. (2018) and Ducrot et al. (2020), at least three planets of the system (TRAPPIST-1b, $d$ and g) are not compatible with a bare rock composition at $1 \sigma$. This stems from the fact that these three planets have a measured bulk density that is lower than a pure $\mathrm{Mg} / \mathrm{Si}$ planet (i.e. the lightest rocky planet that can be constructed), indicated by the black line in Fig. 8. If confirmed, this result is a major achievement because it shows that these three planets must be enriched with volatiles and most likely with water (the most abundant of them) to lower the planetary density.

Dorn et al. (2018) showed using a Bayesian inference analysis that possible water mass fractions may range from 0 to $25 \%$. More recently, Turbet et al. (2020a) showed that the water content of the three innermost planets TRAPPIST-1b, $\mathrm{c}$ and $\mathrm{d}$ may in fact have been overestimated. This stems from the fact that these planets receive more irradiation than the runaway greenhouse limit (see Fig. 1) for which water has been shown to be unstable in condensed form and should rather form a thick, extended $\mathrm{H}_{2} \mathrm{O}$-dominated atmosphere. For the planet to exhibit the same radius, this $\mathrm{H}_{2} \mathrm{O}$-dominated atmosphere needs to be much less massive than a denser condensed water envelope (Turbet et al. 2019a, 2020a). Taking into account this atmospheric effect, Turbet et al. (2020a) showed that TRAPPIST-1b, c and d should not have more (assuming a terrestrial core composition) than 2, 0.3 and $0.08 \%$ of water, respectively.

Bourrier et al. (2017a) investigated - using the formalism of Bolmont et al. (2017a) the history of hydrodynamic water loss from the planets in the energy-limited regime. They found that planet $\mathrm{g}$ and those closer in could have lost more than 20 Earth oceans through hydrodynamic escape, if the system is as old as $\sim 8$ Gigayear (Burgasser and Mamajek 2017). However, TRAPPIST-1e to h might have lost less than three Earth oceans if hydrodynamic escape stopped once they entered the habitable zone, i.e. when diffusion of water to the upper atmosphere (and then photodissociation of water) becomes the limiting process of water loss. Late-stage outgassing could also have brought significant amounts of water for the outer planets after they entered the Habitable Zone. Altogether, this indicates that, while the three inner planets are likely dry today, the outer planets of the TRAPPIST-1 system may have retained some water in their atmosphere or on their surface today.

\section{$5.3 \mathrm{O}_{2}$ Atmospheres}

$\mathrm{O}_{2}$ is the natural molecule to discuss after $\mathrm{H}_{2} \mathrm{O}$ because it has been shown that $\mathrm{O}_{2}$ could easily accumulate from the photodissociation of $\mathrm{H}_{2} \mathrm{O}$ molecules and the subsequent loss of lighter $\mathrm{H}$ atoms.

Luger and Barnes (2015) calculated that as much as $10^{3}-10^{4}$ bar of $\mathrm{O}_{2}$ could have possibly accumulated in the atmosphere of planets orbiting very low mass stars such as TRAPPIST-1. However, the exact amount of $\mathrm{O}_{2}$ that may accumulate in the atmosphere of TRAPPIST-1 planets depends on:

1. how much water is present initially. Tian (2015) showed that the more water is present initially on the planet, the more $\mathrm{O}_{2}$ should build up on the planet. This means in particular 
CONDENSED WATER

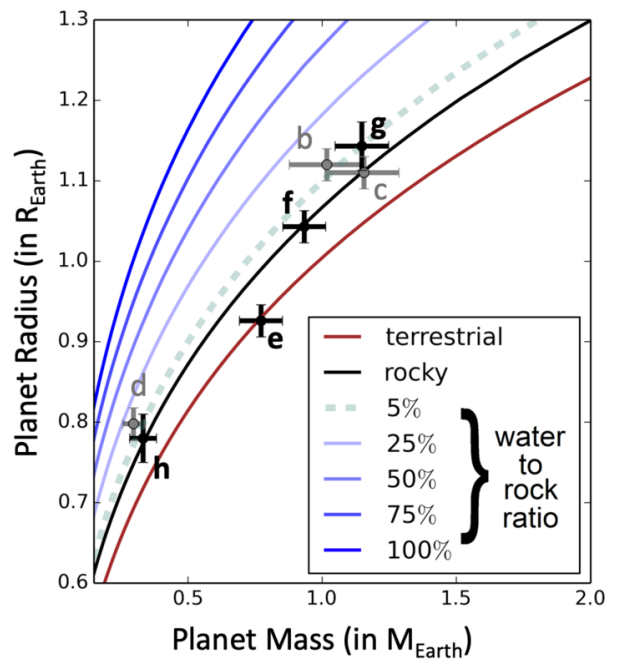

STEAM ATMOSPHERE

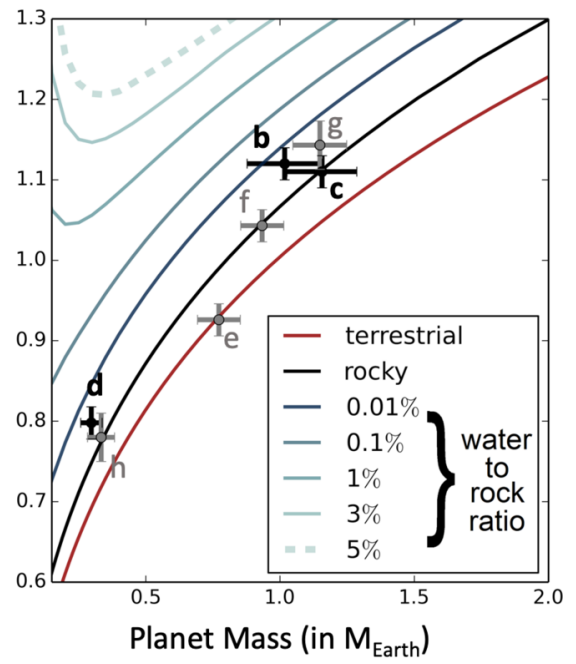

Fig. 8 Mass-radius relationships for various interior compositions and water content, assuming water is in the condensed form (left panel) and water forms an atmosphere (right panel). The rocky composition mass-radius relationship assumes a pure $\mathrm{MgSiO}_{3}$ interior and was taken from Zeng et al. (2016). The water-rich mass-radius relationships for water in condensed form (left panel) were derived using the data from Zeng et al. (2016). The water-rich mass-radius relationships for water in gaseous form (right panel) were calculated in Turbet et al. (2020a). All mass-radius relationships with water were built assuming a pure $\mathrm{MgSiO}_{3}$ interior. For comparison, we added the measured positions of the seven TRAPPIST-1 planets measured from Grimm et al. (2018), Ducrot et al. (2020), with their associated $1 \sigma$ error bars. Based on the irradiation they receive compared to the theoretical runaway greenhouse limit (see Fig. 7), TRAPPIST-1e, f, g and h should be compared with mass-radius relationships on the left, while TRAPPIST-1b, $\mathrm{c}$ and $\mathrm{d}$ should be compared with those on the right. To emphasize this, we indicated on each panel in black the planets (and their associated $1 \sigma$ error bars) for which mass-radius relationships (with water) are appropriate. In contrast, we indicated on each panel in grey the planets (and their associated $1 \sigma$ error bars) for which mass-radius relationships (with water) are not appropriate. For reference, we also added a terrestrial composition that ressembles that of the Earth. Note that mass-radius relationships for steam planets (right panel) can be easily built following the procedure described in Appendix D of Turbet et al. (2020a). The figure was adapted from Turbet et al. (2020a)

that if the TRAPPIST-1 planets formed dry, then the accumulation of $\mathrm{O}_{2}$ should be very limited. This assumes no atmosphere-interior interactions.

2. how efficiently hydrogen atoms can drag oxygen atoms with them. Bolmont et al. (2017a) found that, in most of the configurations of their calculations, oxygen atoms are dragged away by the escaping hydrogen atoms, thus reducing the $\mathrm{O}_{2}$ buildup to hundreds of bar, at maximum (see also Johnstone 2020). This is about two orders of magnitude lower than calculated in Luger and Barnes (2015). Furthermore, dragged along oxygen atoms could cool the outflows, resulting in reduced escape rates.

3. how efficient atmosphere-interior interactions are. Wordsworth et al. (2018) showed that during the runaway greenhouse phase where all water of the planet is trapped in the atmosphere, the surface temperature due to the greenhouse effect of water is so high (Kopparapu et al. 2013b; Goldblatt et al. 2013; Turbet et al. 2019a) that the surface and mantle are expected to melt. In this case, most oxygen produced from $\mathrm{H}_{2} \mathrm{O}$ photolysis can be absorbed by the magma ocean, thus limiting the $\mathrm{O}_{2}$ atmospheric buildup (Hamano et al. 2013; Schaefer et al. 2016; Wordsworth et al. 2018). In the scenarios of Wordsworth et al. (2018), $\mathrm{O}_{2}$ buildup is maximum if the water content is low enough that the planet 
reaches the point where (i) it is beyond the runaway greenhouse meaning all water is vaporized, but (ii) it has low enough water (i.e. roughly 1 Earth water ocean content) that the surface is solidified and cannot absorb $\mathrm{O}_{2}$ anymore. While in this worst case scenario, no more than $\sim 10^{2}$ bar of $\mathrm{O}_{2}$ should build up, in most cases Wordsworth et al. (2018) show the $\mathrm{O}_{2}$ buildup should be limited to 1 bar (50 bar, respectively) for TRAPPIST-1h (TRAPPIST-1b, respectively). Finally, Way M. J. and Del Genio (2020) recently proposed that the surface of a planet may not even need to be a magma ocean for large quantities of $\mathrm{O}_{2}$ to be absorbed. $\mathrm{O}_{2}$ may be indeed absorbed by magma released through large scale resurfacing processes as seen on Venus in the past several hundred million years.

While there are multiple theoretical uncertainties in the above dependencies that have yet to be fully worked out, the conclusion of these works is that $\mathrm{O}_{2}$-dominated atmospheres are one of the most serious candidates for the composition of TRAPPIST-1 planetary atmospheres (Lincowski et al. 2019).

\section{$5.4 \mathrm{CH}_{4} / \mathrm{NH}_{3}$ Atmospheres}

In the same way that $\mathrm{H}_{2} \mathrm{O}$ is expected to be efficiently photodissociated in a water-dominated atmosphere, the photodissociation of $\mathrm{CH}_{4}$ and $\mathrm{NH}_{3}$ (which are not sensitive to the condensation cold trap at the radiation levels received on the TRAPPIST-1 planets; see Turbet et al. 2018) by UV radiation may play a significant role in shaping the TRAPPIST-1 planet atmospheres. High energy cross-section of $\mathrm{CH}_{4}$ peaks around $80 \mathrm{~nm}$ and is significantly higher in the $20-150 \mathrm{~nm}$ wavelength range than for wavelengths just beyond $150 \mathrm{~nm}$ by at least 6 orders of magnitude (Keller-Rudek et al. 2013; Arney et al. 2017). In the 20-150 nm wavelength range, the emission of the star TRAPPIST-1 is much stronger than the Sun (see Fig. 2), relative to their total bolometric emission. This indicates that the photodissociation rate of $\mathrm{CH}_{4}$ (with a photodissociation energy threshold $\sim 277 \mathrm{~nm}$ ) is expected to be very strong around a star like TRAPPIST-1.

It is, for example, estimated that it should take roughly $10 \mathrm{My}$ for Titan to remove all the methane (0.07 bar) from the atmosphere (Yung et al. 1984) and that as much as $~ 30$ bar could have been photochemically removed in the last 4 billion years. If the photodissociation rate scales linearly with the incoming X/EUV flux, as much as $10^{2}-10^{4}$ times (averaged over the surface) more methane could have been photochemically destroyed in the atmosphere of TRAPPIST-1 planets (Turbet et al. 2018). Over the expected age of the system of $7.6 \pm 2.2$ Gy (Burgasser and Mamajek 2017), the planets could have lost between $\sim 120$ bar -Titan's limit, including the gravity correction- and $10^{6}$ bar of $\mathrm{CH}_{4}$-when scaling linearly the $\mathrm{CH}_{4}$ loss with the expected X/EUV flux history on TRAPPIST-1b-through (i) photolysis, then (ii) organic haze formation, and (iii) haze sedimentation on the surface, light hydrogen being lost to space in the process. This mechanism is known as the photochemical atmospheric collapse (Lorenz et al. 1997; Turbet et al. 2018).

Knowing that the $\mathrm{NH}_{3}$ high-energy absorption cross-section also peaks around $80 \mathrm{~nm}$ and is significantly higher in the $20-150 \mathrm{~nm}$ wavelength range (Keller-Rudek et al. 2013), and that the amplitude of the cross-section is similar to that of $\mathrm{CH}_{4}$ (peak at $5 \times 10^{-17} \mathrm{~cm}^{2}$ molecule ${ }^{-1}$ for $\mathrm{CH}_{4}$ versus $3 \times 10^{-17} \mathrm{~cm}^{2}$ molecule ${ }^{-1}$ for $\mathrm{NH}_{3}$, around $80 \mathrm{~nm}$ and at room temperature), the same photochemical atmospheric collapse mechanism should operate for $\mathrm{NH}_{3}$. Unlike $\mathrm{CH}_{4}, \mathrm{NH}_{3}$ (with a photodissociation energy threshold $\sim 301 \mathrm{~nm}$ ) absorption cross-section is also high in the 160-210 nm wavelength range (Keller-Rudek et al. 2013). This means that - comparatively to planets orbiting Sun-like star-the $\mathrm{NH}_{3}$ photochemical atmospheric collapse could be weaker. 
Sustaining continuously a $\mathrm{CH}_{4}$-rich (or $\mathrm{NH}_{3}$-rich) atmosphere over TRAPPIST-1 lifetime would require an extremely large source of methane (or ammonia, respectively). Low concentration of $\mathrm{CH}_{4}$ (up to the $\sim 0.3 \%$ level) could be sustained in TRAPPIST-1 planetary atmospheres assuming Earth-like $\mathrm{CH}_{4}$ surface production rate from the Earth biosphere (Rugheimer et al. 2015a). $\mathrm{CH}_{4}$ production rates would however have to be extremely high to maintain a $\mathrm{CH}_{4}$-dominated atmosphere. A similar argument could be made with regard to $\mathrm{NH}_{3}$ (Kasting 1982). We do, however, acknowledge that the formation of high-altitude organic hazes from the $\mathrm{NH}_{3}$ and/or $\mathrm{CH}_{4}$ photolysis (Sagan and Chyba 1997; Wolf and Toon 2010; Arney et al. 2016) may shield these molecules. This could reduce the $\mathrm{CH}_{4}$ and/or $\mathrm{NH}_{3}$ photolysis rate, which would increase their lifetime.

Similarly, large quantities of $\mathrm{N}_{2}$ could be photodissociated, forming $\mathrm{HCN}$ (Liang et al. 2007; Tian et al. 2011; Krasnopolsky 2009, 2014) and could be lost subsequently in long nitrogen-enriched carbonated chains that could sediment on the surface. This mechanism could in principle remove efficiently $\mathrm{N}_{2}$ from the atmosphere in the long term (Turbet et al. 2018). Coupled photochemical Global Climate Models (see e.g. Chen et al. 2019) could be used in the future to further explore these photochemical atmospheric collapse mechanisms to understand when and to what extent it should play a role in shaping TRAPPIST-1 planetslike atmospheres.

\section{$5.5 \mathrm{~N}_{2}$ Atmospheres}

If it is not lost in a chemically reduced atmosphere, $\mathrm{N}_{2}$ could also be lost because of ion escape mechanisms.

Dong et al. (2018) carried out numerical simulations to characterize the stellar wind produced by TRAPPIST-1 and the resulting atmospheric ion escape rates for all of the seven planets, assuming $\mathrm{CO}_{2}$-dominated atmospheres. In their calculations, the stellar wind-driven atmospheric escape rate ranges from 0.1 to 10 bar per billion year, depending on the planet considered (T1b versus T1h). This number could be lowered by up to two orders of magnitude when considering the planet has a strong magnetic field (Dong et al. 2017).

Dong et al. (2019) showed with the case of TRAPPIST-1g that the rate of atmospheric escape due to stellar wind can actually increase by a factor of $\sim 100$ for $\mathrm{O}_{2}$-dominated atmospheres, since $\mathrm{O}_{2}$-dominated exospheres are not expected to cool as efficiently as $\mathrm{CO}_{2}$ dominated ones. Although to the best of our knowledge no numerical experiments have yet been conducted to study the stellar wind-driven atmospheric escape rate for $\mathrm{N}_{2}$-dominated atmospheres around low mass stars, we argue that quantitatively similar results are expected. $\mathrm{O}_{2}$ and $\mathrm{N}_{2}$ have different structural, electronic and radiative properties (Fennelly and Torr 1992; Heays et al. 2017; Gordon et al. 2017), resulting in a much higher concentration of O+ than that of N+ in the present-day Earth's upper atmosphere (Bilitza et al. 2017). However, Tian et al. $(2008 \mathrm{a}, \mathrm{b})$ showed in fact with a thermosphere model that $\mathrm{N}+$ and $\mathrm{O}+$ ions are expected to be present in quite similar abundance in the upper atmosphere of Earth-like (i.e. $\mathrm{N}_{2} / \mathrm{O}_{2}$-dominated) planetary atmospheres, assuming that they are exposed to large ExtremeUV $(E U V)$ fluxes typical of the young Sun or low-mass stars. As a matter of fact, while the upper atmosphere density of $\mathrm{N}+$ in their model is always at least two orders of magnitude lower than that of $\mathrm{O}+$ for a present-day Earth incident EUV flux, the density of $\mathrm{N}+$ is very close (and always at least $2.5 \times$ lower) to that of $\mathrm{O}+$ for a $10 \times$ higher incident EUV flux (see Tian et al. 2008b, Fig. 7). Using the prescriptions of Tian et al. (2008a), Lichtenegger et al. (2010) evaluated that the stellar wind-driven atmospheric escape rate ranges from 20 to 500 bar of $\mathrm{N}_{2}$ lost per billion year for a planet with an Earth-like atmosphere and exposed to an Extreme-UV from 7 to 20 times that of present-day Earth. 
Although the presence of additional coolant gases such as $\mathrm{CO}_{2}$ may lower these rates (Lichtenegger et al. 2010; Johnstone et al. 2018), we conclude that while an Earth-like atmosphere (i.e. $\sim 1$ bar $\mathrm{N}_{2}$-dominated atmosphere) may be stable against stellar wind-driven escape for the outermost planets of the TRAPPIST- 1 system in the event that $\mathrm{CO}_{2}$ is abundant, this is unlikely to be the case for the inner planets in the system. We acknowledge however that assessing the amount of nitrogen that may have been lost through this nonthermal escape process deserves to be studied in more details with models adapted to the TRAPPIST-1 system (see e.g. Dong et al. 2019).

\section{6 $\mathrm{CO}_{2}$ Atmospheres}

$\mathrm{CO}_{2}$ is the most widespread molecule in the atmospheres of the terrestrial planets of the solar system, and is one of the most likely gases to accumulate in the atmospheres of TRAPPIST-1 planets (Lincowski et al. 2019).

The strongest argument for the presence (and possibly the accumulation) of $\mathrm{CO}_{2}$ resides in its robustness to atmospheric escape processes. $\mathrm{CO}_{2}$ is a very good radiative coolant of upper atmospheres (Tian 2009; Johnstone et al. 2018) due mostly to strong absorption bands (or emission bands, according to the Kirchhoff's law of radiation) in the thermal infrared wavelengths. This radiative cooling, coupled with a heavy molecular weight, strongly reduces the efficiency of thermal escape. In addition, simulations of Dong et al. (2017, 2018, 2020) show $\mathrm{CO}_{2}$ should be rather robust to ion escape mechanisms too.

For the outer TRAPPIST-1 planets (efgh), $\mathrm{CO}_{2}$ accumulation could be limited by surface condensation, which could be particularly strong if the planets were to be locked in synchronous rotation (see Sect. 3.4). Turbet et al. (2018) explored this possibility with 3D Global Climate Model simulations taking into account $\mathrm{CO}_{2}$ surface and atmospheric condensation (see Fig. 9). Specifically, they showed that the $\mathrm{CO}_{2}$ surface collapse mechanism must be all the more important as the planet considered is far from the host star TRAPPIST-1, and the amount of non-condensable gas (that transport heat efficiently; and broaden the absorption lines of $\mathrm{CO}_{2}$, which increases the greenhouse effect) is low.

Recently, Hu et al. (2020) used 1-D atmospheric photochemistry models of TRAPPIST-1 planets assuming $\mathrm{CO}_{2}$-rich atmospheres to show the photodissociation of $\mathrm{CO}_{2}$ may lead to the accumulation of $\mathrm{CO}$ and $\mathrm{O}_{2}$, in agreement with previous calculations (Gao et al. 2015), because the recombination of $\mathrm{CO}$ and $\mathrm{O}$ would be slow around low mass stars such as TRAPPIST-1. While in Mars and Venus catalytical cycles recombine $\mathrm{CO}$ and $\mathrm{O}_{2}$ into $\mathrm{CO}_{2}$ (and thus stabilize $\mathrm{CO}_{2}$ ), Hu et al. (2020) showed that this may not be the case for planets orbiting a very low mass star like TRAPPIST-1. This stems from the fact (Hu et al. 2020) that the photodissociation rate of $\mathrm{CO}_{2}$ is higher (due to increased far-UV emission) and catalytical cycles are less efficient (due to decreased near-UV emission) to reform $\mathrm{CO}_{2}$. We note that while $\mathrm{CO}$ and $\mathrm{O}_{2}$ are not radiatively significant greenhouse gases, the build up of multiple bars of these gases can warm the planet through pressure broadening of remaining $\mathrm{CO}_{2}$, and through adiabatic heating (see Table 4 in Lincowski et al. 2018).

Depending on the water vapour abundance in the atmosphere, and thus whether a liquid water ocean is present or not ( $\mathrm{CO}$ and $\mathrm{O}_{2}$ could also be recombined directly in the ocean), $\mathrm{CO}$ and $\mathrm{O}_{2}$ could potentially massively accumulate in a $\mathrm{CO}_{2}$-rich planetary atmosphere around TRAPPIST-1 (Gao et al. 2015; Hu et al. 2020). 

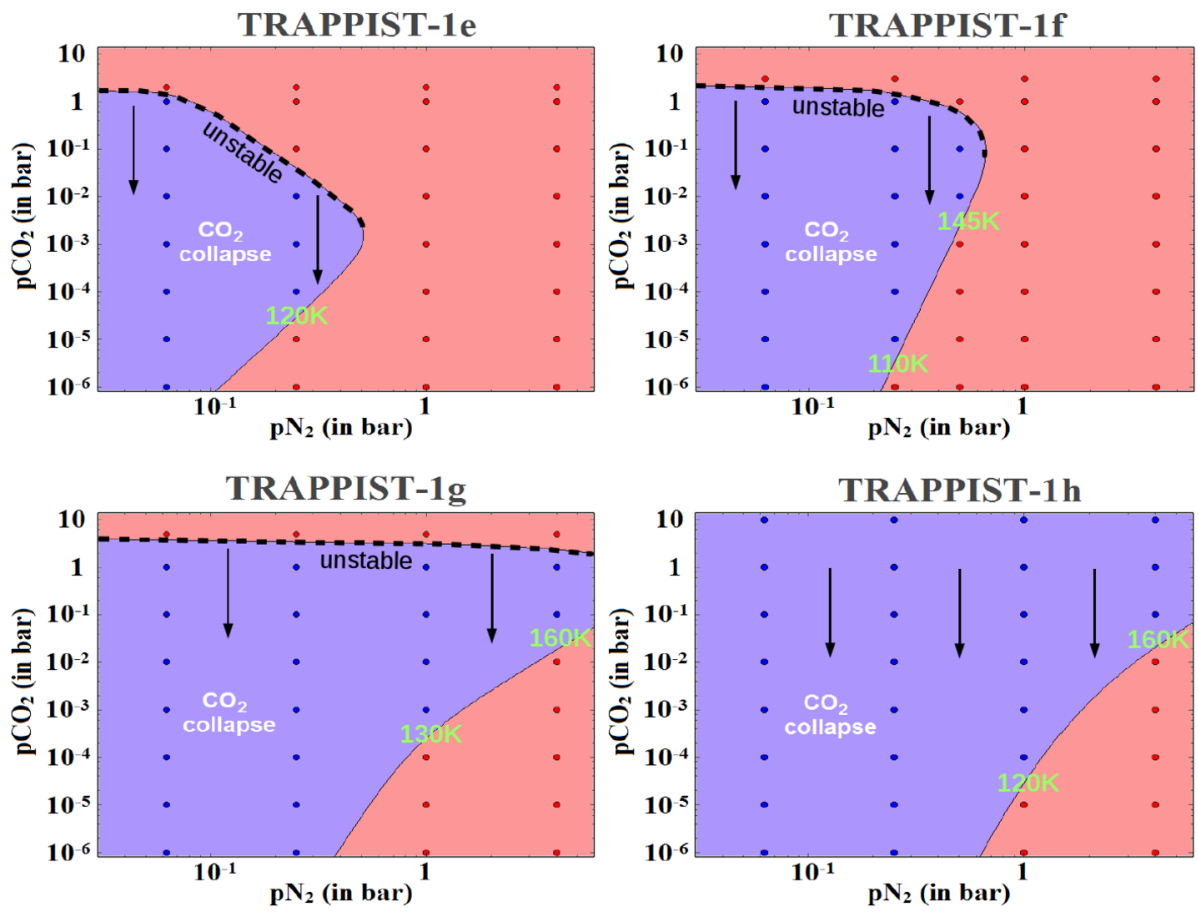

Fig. 9 This diagram indicates the range of $\mathrm{N}_{2}$ and $\mathrm{CO}_{2}$ partial pressures for which TRAPPIST-1 planetary atmospheres are robust to $\mathrm{CO}_{2}$ surface condensation collapse (red regions) or not (blue regions). Each dot corresponds to the result of a 3-D Global Climate Model simulation. The black arrows indicate how planets that have an unstable atmosphere (due to $\mathrm{CO}_{2}$ surface condensation) would evolve on the diagram. Temperatures (in green) correspond to the rough estimate (based on GCM simulations) of the surface temperature of the coldest point of the planet, at the stable lower boundary (blue is up; red is down). The figure was taken from Turbet et al. (2018)

\subsection{Implications for the Presence of Surface Liquid Water on TRAPPIST-1 Planets}

Several 3-D Global Climate Models have been used to explore the potential habitability of TRAPPIST-1 planets, i.e. their ability to sustain liquid water on their surface. In short, and looking at Fig. 1 we see that there are three main possible scenarios

- Planets b and c (and potentially d) receive more insolation than the runaway greenhouse limit for water. A global surface ocean is therefore unstable, resulting in steam atmospheres and the eventual loss of water to space, pushing the planets into a desiccated state. The presence of surface liquid water would require some very specific circumstances, such as a very thin atmosphere with surface liquid water trapped on the nightside, assuming they are locked in synchronous rotation (Leconte et al. 2013; Turbet et al. 2016, 2018).

- Planets e, $\mathrm{f}$ and $\mathrm{g}$ are in a region where only moderate amounts of $\mathrm{CO}_{2}$ would suffice to warm the surface above the melting point of water (Wolf 2018; Turbet et al. 2018; Fauchez et al. 2019). TRAPPIST-1e, in particular, should be able to sustain surface liquid water for a wide range of atmospheric compositions and pressures (Turbet et al. 2018). Note presently underway is the TRAPPIST-1 Habitable Atmosphere Intercomparison (THAI) 


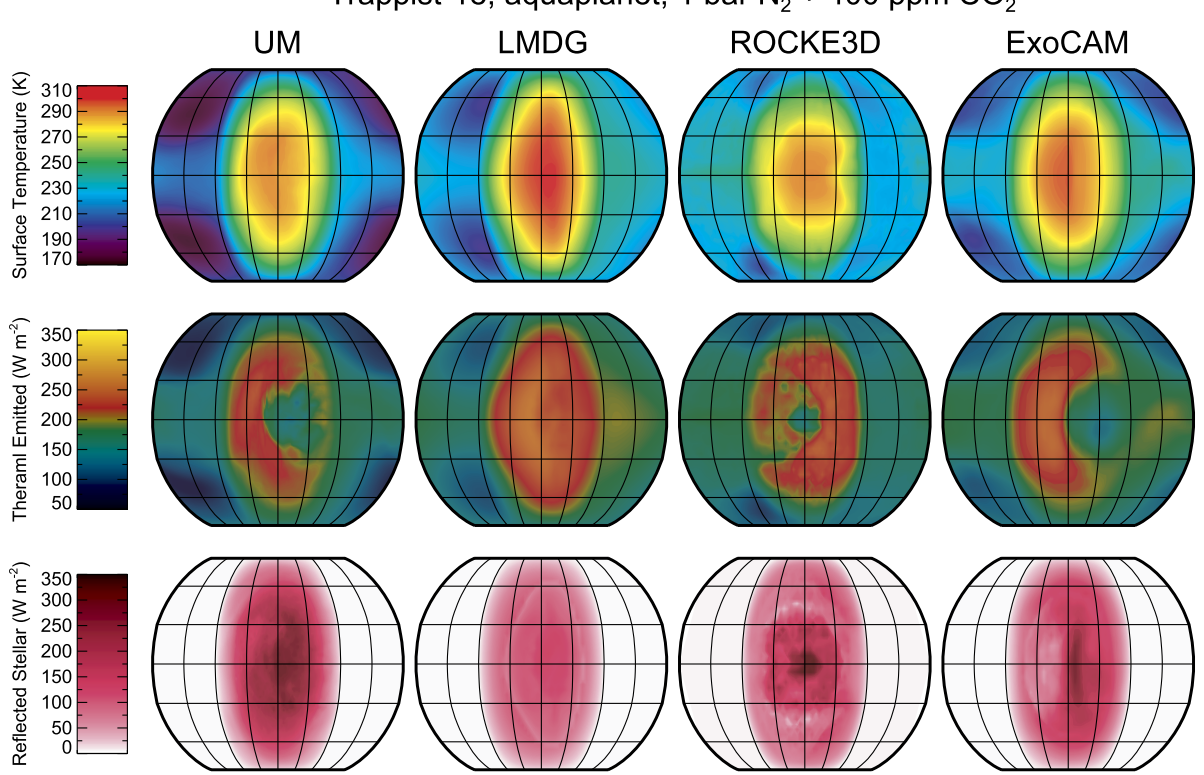

Fig. 10 Surface contours for surface temperature, thermal emitted radiation at the top of the atmosphere (TOA) and reflected stellar radiation at TOA for "Hab1" scenario (i.e. a scenario of a planet with global ocean and an atmospheric bulk composition similar to present-day Earth) simulated by four of the state-of-the-art exoplanet GCMs: the UK Met Office United Model (UM) (Mayne et al. 2014; Boutle et al. 2017), the Laboratoire de Météorologie Dynamique Generic model (LMDG) (Wordsworth et al. 2011; Turbet et al. 2018), the Resolving Orbital and Climate Keys of Earth and Extraterrestrial Environments with Dynamics (ROCKE-3D) (Way et al. 2017), and the National Center for Atmospheric Research Community Atmosphere Model version 4 modified for exoplanets (ExoCAM) (Wolf and Toon 2015; Wolf 2017). The figure was taken from Fauchez et al. (2020b)

project (Fauchez et al. 2020b), where multiple 3-D GCMs will be compared for habitable iterations of TRAPPIST-1e. This is illustrated in Fig. 10. While differences in the cloud fields produce variations in the thermal emitted and reflected stellar fluxes, all GCMs tested similarly predict temperate surface conditions and large areas of open ocean on the day-side of the planet, assuming an Earth-like atmospheric composition.

- Planet $\mathrm{h}$ is further out where the classical $\mathrm{CO}_{2}$ runaway collapse is supposed to take place. To reach temperate conditions, in addition to a very thick $\mathrm{CO}_{2}$ atmosphere, the planet would need to retain enough volcanic gases such as $\mathrm{CH}_{4}$ or $\mathrm{H}_{2}$ in order to block $\mathrm{CO}_{2}$ infrared windows and prevent the collapse of the atmosphere (Pierrehumbert and Gaidos 2011; Wordsworth et al. 2017; Ramirez and Kaltenegger 2017; Lincowski et al. 2018; Turbet et al. 2018, 2019b, 2020b).

A detailed discussion about the potential habitability of TRAPPIST-1 planets is provided in Wolf $(2017,2018)$ as well as in the Sect. 7 of Turbet et al. (2018).

\section{Future Prospects}

Thanks to its exceptional properties (system very close to Earth, extremely small host star, planets transiting frequently, very compact orbital architecture), the TRAPPIST-1 system 
and its seven temperate-orbit, terrestrial-size planets is likely our best chance in the future to learn more about potentially habitable planets. There are several ways to learn more about the system and its planets in the future.

First of all, the basic properties of the seven planets (i.e. their masses and radii) need to be determined with more accuracy in order to carry out comparative planetology with high-precision density measurements. Mass measurements of TRAPPIST-1 planets could be improved with the help of a TTV analysis (i) including all existing transit light curves (e.g. all Spitzer light curves, which are the best quality data available so far; see Agol et al. 2020, in preparation) and accounting for subtle processes such as gravitational tides (that may influence TTV in such a compact system; see Bolmont et al. 2020) and for the presence of additional non-detected (yet!) planets in the system. Mass measurements could also be obtained with radial velocity measurements with near-infrared spectrographs mounted on big telescopes such as SPIRou (Klein and Donati 2019), CARMENES (Quirrenbach et al. 2014), IRD (Kotani et al. 2018) and NIRPS (Wildi et al. 2017). Near infrared wavelengths are optimal for these observations because this is where TRAPPIST-1 is the brightest. Eventually, the best mass measurements could be derived using a joined fit of the TTV measurements, the RV measurements, and the long-term stability of the system.

Likewise, radius measurements of TRAPPIST-1 planets could be improved through several ways. First, a more accurate measurement of the stellar radius will help to better constrain the planet's radii because their transit depths measure the planet-to-star radius ratios. TRAPPIST-1 radius has been measured to a $\sim 3 \%$ precision (at $1 \sigma$ ) so far (Van Grootel et al. 2018). The same $\sim 3 \%$ uncertainty propagates therefore on the absolute radii of the planets. The distance of the star TRAPPIST-1 has been measured-thanks to Gaia DR2 parallaxes - to the exquisite accuracy of $0.16 \%$ (Gaia Collaboration et al. 2018; Lindegren et al. 2018; Kane 2018), which is low enough that it has a very minor impact on the final radii uncertainty. The $\sim 3 \%$ stellar uncertainty mostly depends now on the uncertainty on the age of TRAPPIST-1 (Burgasser and Mamajek 2017), which propagates to stellar radius uncertainty in stellar evolution models such as those of Filippazzo et al. (2015). However, we acknowledge that the density should be better constrained than either the mass or radius, because it can be computed without the stellar uncertainty (Grimm et al. 2018). Note that the stellar radius estimate may also be biased by (i) magnetic activity effects and/or (ii) tidal interactions of the planets with the star (Burgasser and Mamajek 2017; Gonzales et al. 2019) which are not included in existing stellar evolution models. Secondly, stellar contamination of the photosphere of TRAPPIST-1 by spots need to be further investigated, as it was shown to be a potential source of bias - up to $\sim 2.5 \%$ for TRAPPIST- 1 in the infrared Spitzer IRAC bands - for the radius estimates (Rackham et al. 2018). Last, alternative methods for planetary radius determination such as the ingress/egress duration measurement (Morris et al. 2018c) could be used on larger datasets to derive independent, possibly more precise measurements.

The second main area of progress is atmospheric characterizations techniques: (i) transmission spectroscopy during transits and (ii) thermal infrared secondary eclipses and phase curves. Transmission spectroscopy observations have been initiated on TRAPPIST-1 planets in the near-infrared with HST/WFC3 observations (de Wit et al. 2016). Meanwhile, significant efforts have been made to prepare transmission spectroscopy observations with forthcoming large-aperture telescopes (Barstow and Irwin 2016; Morley et al. 2017; Lincowski et al. 2018; Krissansen-Totton et al. 2018; Wunderlich et al. 2019; Lustig-Yaeger et al. 2019; Fauchez et al. 2019; Pidhorodetska et al. 2020) with a particular focus on the James Webb Space Telescope (Gillon et al. 2020) which should be in operation within a few years. Morley et al. (2017) first used a very simplified reverse 1-D radiative-convective 

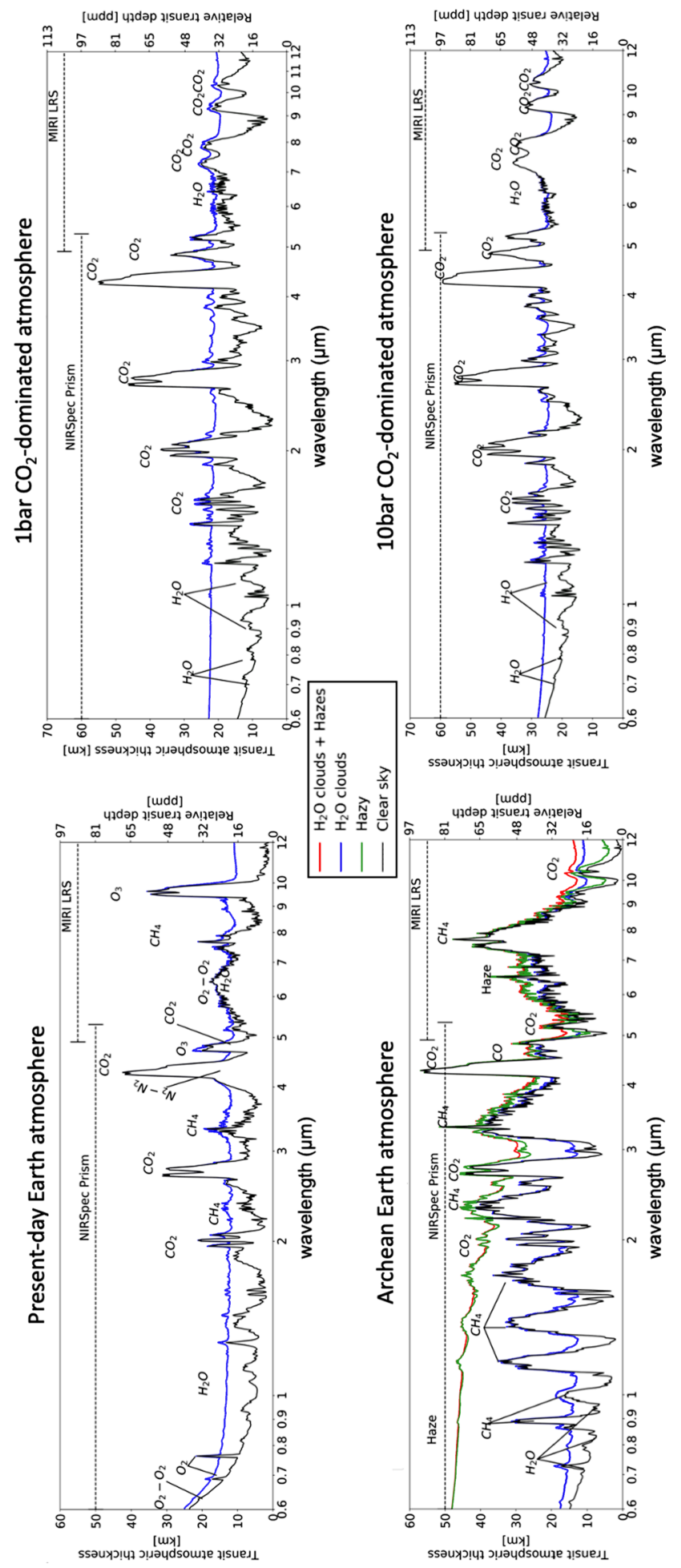

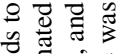

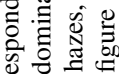

웡 월

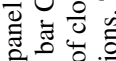

중 的政

Iิ

훈

定

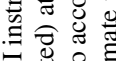

플의

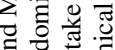

U

远: 西

त

Z

는하요

政

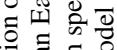

政它

o

表

\&

एँ

ठ용

त्ज ज ले

㯊

고을

के

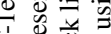

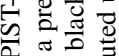

言芒。

政

के 0

농

응

䒕嘅

के

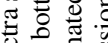

ญํㅣㄹ.

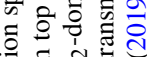

ह

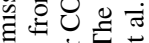

和藏

폽을응

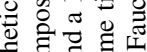

司寻

六艺氙导

=

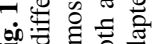

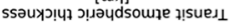

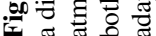


model of TRAPPIST-1 planets to determine our ability to characterize Earth-like, Venus-like and Titan-like atmospheres around TRAPPIST-1 planets. They found that $\sim 20$ transits are required for a $5 \sigma$ detection of molecular spectral features (i.e. to rule out a flat line at $5 \sigma$ confidence) for most of TRAPPIST-1 planets, but could be as low as 4 transits for some planets if their properties are favourable. Lincowski et al. (2018) then used a 1-D radiative-convective model coupled with a photochemistry module to show that transit spectroscopy on JWST can be used to distinguish between some of the possible atmospheres (e.g. $\mathrm{CO}_{2}$-dominated and $\mathrm{O}_{2}$-dominated, discussed in Sect. 5) expected on TRAPPIST-1 planets. Using the same models, Lustig-Yaeger et al. (2019) estimated that $\mathrm{CO}_{2}$-containing atmospheres could be detected potentially in fewer than 10 transits - for all seven TRAPPIST-1 planets-if they lack high-altitude aerosols. Fauchez et al. (2019) calculated similar number of transits using more sophisticated 3-D Global Climate Models. The detection of $\mathrm{CO}_{2}$-containing atmosphere can be done mainly through the strong $\mathrm{CO}_{2} 4.3 \mu \mathrm{m}$ absorption band (Barstow and Irwin 2016; Morley et al. 2017; Krissansen-Totton et al. 2018; Lustig-Yaeger et al. 2019; Wunderlich et al. 2019; Fauchez et al. 2019; Pidhorodetska et al. 2020). This $4.3 \mu \mathrm{m} \mathrm{CO}_{2}$ band is clearly the most promising absorption feature to search for in TRAPPIST-1 planets transmission spectra with JWST. As illustrated in Fig. 11, it is weakly affected by clouds and hazes and robust to a wide range of $\mathrm{CO}_{2}$ concentration. Water may be extremely difficult to detect for TRAPPIST-1e and more distant planets (unless the planets are in an unlikely moist greenhouse state) because (i) water vapour should be-in most cases-confined in the lower atmosphere (because of a condensation cold trap at the top of the troposphere) where transmission spectra are less sensitive (Lustig-Yaeger et al. 2019; Fauchez et al. 2019), but also because (ii) water clouds (forming preferentially at the top of the troposphere) should flatten the transmission spectra (Fauchez et al. 2019; Komacek et al. 2020). $\mathrm{O}_{2}$ could also be detected with transit spectroscopy on JWST through the $\mathrm{O}_{2}-\mathrm{O}_{2}$ infrared $6.4 \mu \mathrm{m}$ collisioninduced absorption (Fauchez et al. 2020a) in $\sim 10$ transits for most planets of the system if they have a very dry $\mathrm{O}_{2}$-dominated atmosphere. The presence of $\mathrm{O}_{2}$ could also be indirectly inferred from the detection of $\mathrm{O}_{3}$ (through the $9.6 \mu \mathrm{m}$ absorption band), which has been shown to be detectable in tens of transits if present at present-day Earth level (Barstow and Irwin 2016). Detecting these two molecular features $\left(\mathrm{O}_{2}-\mathrm{O}_{2}\right.$ at $6.4 \mu \mathrm{m} ; \mathrm{O}_{3}$ at $\left.9.6 \mu \mathrm{m}\right)$ requires the use of the JWST-MIRI ${ }^{7}$ (Mid-InfraRed Instrument) instrument (Rieke et al. 2015) in Low Resolution Spectroscopy (LRS) mode (Kendrew et al. 2015). The analysis of Fauchez et al. (2019) suggests that other gases would potentially require hundreds (or thousands) of transits to be detectable, because of clouds and/or hazes flattening the transmission spectra in near-infrared wavelengths. Lincowski et al. (2019) proposed that JWSTNIRSpec $^{8}$ (Near-InfraRed Spectrograph) in particular in prism mode (Bagnasco et al. 2007; Ferruit et al. 2014) transmission spectra could be used to detect $\mathrm{CO}_{2}$ and $\mathrm{H}_{2} \mathrm{O}$ isotopologues, which would be very informative about isotopic fractionation processes such as atmospheric escape. Isotopologues such as $\mathrm{HDO}$ or ${ }^{18} \mathrm{OCO}$ (at mixing ratios compatible with solar system values) could be detected through their near-infrared absorption bands with as few as 4 to 11 transits at $5 \sigma$ on JWST-NIRSpec Prism (Lincowski et al. 2019). However, the presence of clouds and/or hazes would likely preclude detection of these isotopologues with

\footnotetext{
${ }^{7}$ MIRI has a wavelength coverage from 4.9 to $28.8 \mu \mathrm{m}$. It can be used with 9 different broad-band filters, and can also be used in a Low Resolution Spectroscopy (LRS) mode of $\mathrm{R}(\lambda / \Delta \lambda) \sim 100$. Detailed documentation is available at https://jwst-docs.stsci.edu/.

${ }^{8}$ NIRSPEC has a wavelength coverage from 0.6 to $5.3 \mu \mathrm{m}$. It can be used in several resolving power modes, including a low resolution prism mode $(\mathrm{R}(\lambda / \Delta \lambda) \sim 30-300)$ and several medium-to-high resolution grism modes $(\mathrm{R}(\lambda / \Delta \lambda) \sim 1000-3000)$ but with a smaller wavelength coverage. Detailed documentation is available at https://jwst-docs.stsci.edu/.
} 
JWST (Fauchez et al. 2019). Moreover, we emphasize that the real impact of stellar contamination by the presence of heterogeneities in the photosphere of TRAPPIST- 1 needs to be elucidated, as it can significantly bias the transmission spectra measured with JWST. For that, more information need to be gathered on TRAPPIST-1 during planetary transits (e.g. search for spot crossing events) and out of planetary transit (e.g. characterize the wavelength dependency of the stellar variability). That being said, these promising results motivated altogether Guaranteed Time Observations (GTO) programs on JWST (GTO 1201, PI: David Lafreniere; GTO 1331, PI: Nikole Lewis) to observe 2 transits of TRAPPIST-1d and 4 of TRAPPIST-1e with NIRSpec prism; 4 transits of TRAPPIST-1f and 3 of TRAPPIST-1g with JWST-NIRISS $^{9}$ (Near InfraRed Imager and Slitless Spectrograph) (Doyon et al. 2012).

In parallel, large-aperture ground-based telescopes coupled to high-resolution spectrographs could be used to infer the properties of the TRAPPIST-1 system and the planetary atmospheres. Such observations could lead to (i) the detection of the Rossiter-McLaughlin effect (Rossiter 1924; McLaughlin 1924; Cloutier and Triaud 2016) to further constrain the orbital architecture of the system and confirm the 3.3 days rotation period of TRAPPIST-1, which is a crucial assumption in the stellar contamination model of Morris et al. 2018a. Hirano et al. (2020) have very recently made a first potential detection of the RossiterMcLaughlin effect with the Subaru-IRD spectrograph and derived a projected rotation velocity of TRAPPIST- 1 of $1.49_{-0.37}^{+0.36} \mathrm{~km} \mathrm{~s}^{-1}$, which corresponds to a maximum stellar rotation period of $3.97_{-0.77}^{+1.32}$ days, in agreement with the 3.3 days rotation period from the K2 light curves. It is also consistent with the stellar line rotational broadening measurement of TRAPPIST-1 with CARMENES (Reiners et al. 2018) leading to a projected rotation velocity of $\sim 2 \mathrm{~km} \mathrm{~s}^{-1}$. The observations of Hirano et al. (2020) also suggest that the stellar obliquity of TRAPPIST-1 (i.e. the angle between the star's spin axis and the orbital axis of the planets) is likely small, providing new information on the orbital architecture of the system.; (ii) the detection of molecular absorption lines. At very high spectral resolution, molecular absorption lines can be individually resolved and their signal can be co-added using the cross-correlation technique (Snellen et al. 2013). The contamination by resolved telluric lines can be significantly reduced by taking advantage of the Doppler shift arising from the differential speed of the observed system and observers on Earth. Specifically, detection of molecules such as $\mathrm{H}_{2} \mathrm{O}$ or $\mathrm{O}_{2}$ (e.g. through the $760 \mathrm{~nm}$ A-band) could be attempted on the E-ELT (Snellen et al. 2013; Rodler and López-Morales 2014; Serindag and Snellen 2019), although the number of required transits may be prohibitively high, especially if clouds and/or hazes are present; (iii) the detection of the high-resolution component (e.g. inverted water vapour feature) of the stellar contamination spectrum (see Fig. 4-5 of Ducrot et al. 2018). The amplitude of inverted water vapour features can be as high as $\sim 1000 \mathrm{ppm}$ per planet and this signal could be again boosted with the use of the cross-correlation technique. These transit observations could be attempted with near-infrared instruments on existing large telescopes (e.g. CFHT-SPIRou, ESO-3.6m-NIRPS, Calar Alto-CARMENES, SubaruIRD) and future instruments on extremely large telescopes (e.g. E-ELT/HIRES); (iv) the use of Doppler tomography techniques to probe the photosphere of TRAPPIST-1 and put constraints on stellar contamination models. For example, the Zeeman Doppler Imaging (ZDI) tomographic technique could be used to characterize the distribution of magnetically active regions at the stellar surfaces (Hébrard et al. 2016) and thus help to identify the nature of stellar heterogeneities of TRAPPIST-1's photosphere, e.g. the distribution of spots. Such

\footnotetext{
${ }^{9}$ NIRISS has a wavelength coverage from 0.6 to $2.8 \mu \mathrm{m}$. In SOSS (Single Object Slitless Spectroscopy) mode, which is the most relevant mode for the characterization of exoplanet atmospheres, it has a medium-resolution ( $\mathrm{R}(\lambda / \Delta \lambda) \sim 150)$. Detailed documentation is available at https://jwst-docs.stsci.edu/.
} 
observations could be attempted with near-infrared spectro-polarimeters such as SPIRou or CRIRES+ (Follert et al. 2014).

In an even more distant future, transit observations in UV could be attempted with UVSPEX, a conceptual design of Ultraviolet Spectrograph for Exoplanet (UVSPEX) for World Space Observatory Ultraviolet (WSO-UV), to detect the presence of an oxygen-rich exosphere (Kameda et al. 2019). TRAPPIST-1 would be right at the limit of capability of the instrument/telescope; and with LUVOIR/LUMOS to detect the presence of an hydrogenrich exosphere in Lyman- $\alpha$ (dos Santos et al. 2019). As a matter of fact, dos Santos et al. (2019) calculated that it would be possible to detect the exosphere of an Earth-like planet transiting TRAPPIST-1 at $5 \sigma$ within 10 transits using LUVOIR-A design with the LUMOS instrument concept.

In parallel, thermal emission observations have been initiated on TRAPPIST-1 planets by observations of the secondary eclipses of TRAPPIST-1b and c using the Spitzer/IRAC 4$5 \mu \mathrm{m}$ channel (Ducrot et al. 2020). While these observations were inconclusive because of the low signal to noise level of the observations (Ducrot et al. 2020), Morley et al. (2017) have shown that JWST-MIRI could bring useful constraints in a handful of secondary eclipses for the innermost planets of the system. Besides, emission spectroscopy and photometry are expected to be less affected by the presence of heterogeneities in the photosphere of TRAPPIST-1. Morley et al. (2017) showed that TRAPPIST-1b - the most irradiated planet of the system-is an excellent target for emission spectroscopy with JWST-MIRI in LRS mode, requiring fewer than 10 eclipse observations to detect the band-integrated thermal emission at a $25 \sigma$ confidence. Inferring the atmospheric composition $\left(\mathrm{CO}_{2}\right.$-dominated? $\mathrm{H}_{2} \mathrm{O}$-dominated? $\mathrm{CH}_{4}$-dominated?) of TRAPPIST-1b may require tens (possibly hundreds) of eclipses for a $5 \sigma$ detection (Morley et al. 2017; Lustig-Yaeger et al. 2019; Koll et al. 2019; Malik et al. 2019), depending on the atmospheric composition and pressure of the planet. These promising results motivated two Guaranteed Time Observations (GTO) programs on JWST (GTO 1279, PI: Pierre-Olivier Lagage; GTO 1177, PI: Thomas Greene) to observe 10 secondary eclipses of TRAPPIST-1b with MIRI in imager mode. TRAPPIST-1c and outer planets which are supposedly colder are likely out of reach of eclipse spectroscopy (Lustig-Yaeger et al. 2019). Eclipse photometry could be used to detect an atmosphere on these colder planets in tens of eclipses (Morley et al. 2017; Lustig-Yaeger et al. 2019), but this requires special circumstances (right combination of JWST-MIRI photometric filters and planet atmospheric compositions).

Last but not least, thermal phase curves (Selsis et al. 2011) could be used to further constrain the presence of an atmosphere on TRAPPIST-1 planets as was recently done on the very hot, rocky exoplanet LHS 3844b (Kreidberg et al. 2019). However, such observations would be time-consuming and potentially difficult to analyze because TRAPPIST- 1 is a multiplanetary system and the measured signal would be the result of the superposition of the thermal emission signal of 7 distinct planets.

\section{Conclusions}

First of all, we have seen that the host star of the system TRAPPIST-1 is rather harmful to the atmospheric evolution of the planets due to (i) the evolution of its luminosity during the long Pre Main Sequence phase likely causing a runaway greenhouse and (ii) its high nonthermal $\mathrm{X}$ and far-UV emission (below $\sim 150 \mathrm{~nm}$ ). These two characteristics put together suggest that the TRAPPIST-1 planets likely suffered from intense atmospheric erosion. 
However, the ability of the planets today to have an atmosphere depends just as much on their initial volatile reservoir, which strongly depends on how they formed. For now, a scenario where planets were formed far away in the protoplanetary disk (and thus possibly beyond the ice lines of common volatile species such as $\mathrm{H}_{2} \mathrm{O}$ ) and then migrated inwards in resonant chains is preferred, mostly because of the orbital architecture of the system (highly compact and resonant). This is of course not the only possible formation scenario, just as it is not the only possible scenario in which the planets would have an atmosphere today (i.e. secondary outgassing or late cometary delivery). It is important to note that all processes (planet formation, stellar evolution, X/UV and stellar-wind driven erosion, cometary delivery, outgassing) should make it easier for the outer, more massive planets in the system to have an atmosphere than the inner planets.

The most favored formation scenario (formation far in the disk combined with resonant inward migration) suggests that TRAPPIST-1 planets may be enriched in volatiles, possibly water, thus possibly lowering their density in a detectable way. Preliminary results combining radius estimates (based on Spitzer transit light curves, and precise stellar parameters for TRAPPIST-1) with mass estimates (based on TTV analysis, and confirmed with orbital stability analysis) show this may be the case for at least some planets in the system.

Near-infrared transit observations with the Hubble Space Telescope show that the six inner planets are unlikely to be endowed with a cloud-free hydrogen-dominated envelope. Although the transit observations cannot exclude the case of hydrogen-dominated envelopes with high altitude clouds, measurements of the mass and radius of the planets, combined with atmospheric escape modelling and gas accretion modelling, indicate altogether that this case is unlikely. Despite many observation campaigns with many different telescopes (Spitzer, Hubble, K2, VLT, etc.), existing transit transmission spectra cannot be used to infer the molecular composition of atmospheres (if any) of TRAPPIST-1 planets. This stems from the fact that (i) the signal to noise ratio of single observations is insufficient to detect high mean molecular weight atmospheres, (ii) it is challenging to accurately compare the absolute transit depths between observations made by different instruments at different wavelengths, (iii) last but not least the transit transmission spectra may be significantly affected by the stellar contamination due to the presence of (occulted and/or unocculted) cold and/or bright spots in the photosphere of TRAPPIST-1. Depending on the (still debated) quantity, temperature(s) and spatial distribution of the spots, stellar contamination may (or may not) have a huge impact on the transit spectra of the TRAPPIST-1 planets. By combining information from all existing observations on the system (stellar temporal variability at different wavelengths, search for spot crossing events in the transit light curves, quality of the fit of the stellar contamination spectrum on the combined transit light curves, etc.) it should be possible to better constrain the nature of heterogeneities present in the photosphere of TRAPPIST-1, as well as to build a stellar contamination model that can be used to correct TRAPPIST-1 planets transit transmission spectra. This work is crucial in preparing future transit observations with the James Webb Space Telescope (Gillon et al. 2020).

In addition to transit observations, density measurements can be used to place constraints on the possible water content of the system's planets. Specifically, possible water mass fractions range from 0 up to $25 \%$, despite expected strong atmospheric water vapour erosion. Upper water mass fraction estimates are however expected to be significantly lowered for TRAPPIST-1 b, c and d. This stems from the fact that on these three planets, water must be in the form of steam, which increases - for a given amount of water-the apparent size of their atmosphere.

Last, numerical modeling (3-D global climate models, 1-D photochemical climate models, ion escape models) can be used to further constrain the possible atmospheres of TRAPPIST-1 planets. Some of the most likely atmospheres include: 
1. $\mathrm{H}_{2} \mathrm{O}$-dominated atmospheres. Only for the three inner TRAPPIST-1 planets, because they are more irradiated than the runaway greenhouse radiation limit.

2. $\mathrm{O}_{2}$-dominated atmospheres. They are the natural remnant of early erosion of $\mathrm{H}_{2} \mathrm{O}$ dominated atmospheres.

3. $\mathrm{CO}_{2}$-dominated atmospheres. This stems from the fact that $\mathrm{CO}_{2}$ is much more resilient to atmospheric escape processes than other common gases. However, photochemistry and surface condensation could significantly reduce the $\mathrm{CO}_{2}$ content of the atmosphere.

$\mathrm{CH}_{4}$ and $\mathrm{NH}_{3}$ are unlikely to be dominant gases because of their sensitivity to atmospheric photochemical collapse. It is unlikely that the planets could have maintained an $\mathrm{N}_{2}$ dominated atmosphere (especially the innermost planets) unless they started with a much higher nitrogen content than that of the present-day Earth.

There are undoubtedly, at the present stage, so many known unknowns and unknown unknowns that we must remain open to a wide range of possible atmospheric compositions of the TRAPPIST-1 planets. Fortunately, a new wave of observations with the James Webb Space Telescope and near-infrared high-resolution ground-based spectrographs on existing very large and forthcoming extremely large telescopes will bring significant advances in the coming decade.

Acknowledgements Open access funding provided by University of Geneva. This project has received funding from the European Union's Horizon 2020 research and innovation program under the Marie Sklodowska-Curie Grant Agreement No. 832738/ESCAPE, and through the European Research Council (ERC) grant agreements $n^{\circ}$ 679030/WHIPLASH and $n^{\circ}$ 724427/FOUR ACES. M.T. thanks the Gruber Foundation for its support to this research. M.T. thanks Sarah Peacock for sharing her high resolution TRAPPIST-1 spectra. M.T. thanks Nathan Hara and Christophe Lovis for useful discussions related to this work. The authors acknowledge support by the Swiss National Science Foundation (SNSF) in the frame of the National Centre for Competence in Research "PlanetS". This research has made use of NASA's Astrophysics Data System. This project is partly supported by the International Space Science Institute (ISSI) in the framework of an international team entitled "Understanding the Diversity of Planetary Atmospheres". The authors thank the reviewers for their constructive remarks and comments which helped to improve the manuscript.

Publisher's Note Springer Nature remains neutral with regard to jurisdictional claims in published maps and institutional affiliations.

Open Access This article is licensed under a Creative Commons Attribution 4.0 International License, which permits use, sharing, adaptation, distribution and reproduction in any medium or format, as long as you give appropriate credit to the original author(s) and the source, provide a link to the Creative Commons licence, and indicate if changes were made. The images or other third party material in this article are included in the article's Creative Commons licence, unless indicated otherwise in a credit line to the material. If material is not included in the article's Creative Commons licence and your intended use is not permitted by statutory regulation or exceeds the permitted use, you will need to obtain permission directly from the copyright holder. To view a copy of this licence, visit http://creativecommons.org/licenses/by/4.0/.

\section{References}

E. Agol, J. Steffen, R. Sari, W. Clarkson, On detecting terrestrial planets with timing of giant planet transits. Mon. Not. R. Astron. Soc. 359, 567-579 (2005)

E. Agol et al., Planet. Sci. J. (2020, submitted for publication)

R.L. Akeson, X. Chen, D. Ciardi, M. Crane, J. Good, M. Harbut, E. Jackson, S.R. Kane, A.C. Laity, S. Leifer, M. Lynn, D.L. McElroy, M. Papin, P. Plavchan, S.V. Ramírez, The NASA exoplanet archive: data and tools for exoplanet research. Publ. Astron. Soc. Pac. 125(930), 989 (2013)

G. Arney, S.D. Domagal-Goldman, V.S. Meadows, E.T. Wolf, E. Schwieterman, B. Charnay, M. Claire, E. Hébrard, M.G. Trainer, The pale orange dot: the spectrum and habitability of hazy Archean Earth. Astrobiology 16, 873-899 (2016) 
G.N. Arney, V.S. Meadows, S.D. Domagal-Goldman, D. Deming, T.D. Robinson, G. Tovar, E.T. Wolf, E. Schwieterman, Pale orange dots: the impact of organic haze on the habitability and detectability of Earthlike exoplanets. Astrophys. J. 836, 49 (2017)

P. Auclair-Desrotour, K. Heng, Atmospheric stability and collapse on tidally locked rocky planets (2020). ArXiv e-prints, arXiv:2004.07134

P. Auclair-Desrotour, J. Laskar, S. Mathis, A.C.M. Correia, The rotation of planets hosting atmospheric tides: from Venus to habitable super-Earths. Astron. Astrophys. 603, A108 (2017)

P. Auclair-Desrotour, J. Leconte, E. Bolmont, S. Mathis, Final spin states of eccentric ocean planets. Astron. Astrophys. 629, A132 (2019)

G. Bagnasco, M. Kolm, P. Ferruit, K. Honnen, J. Koehler, R. Lemke, M. Maschmann, M. Melf, G. Noyer, P. Rumler, J.-C. Salvignol, P. Strada, M. Te Plate, Overview of the near-infrared spectrograph (NIRSpec) instrument on-board the James Webb Space Telescope (JWST), in Society of Photo-Optical Instrumentation Engineers (SPIE) Conference Series, vol. 6692, (2007), p. 66920M

I. Baraffe, G. Chabrier, F. Allard, P.H. Hauschildt, Evolutionary models for solar metallicity low-mass stars: mass-magnitude relationships and color-magnitude diagrams. Astron. Astrophys. 337, 403-412 (1998)

I. Baraffe, D. Homeier, F. Allard, G. Chabrier, New evolutionary models for pre-main sequence and main sequence low-mass stars down to the hydrogen-burning limit. Astron. Astrophys. 577, A42 (2015)

A.C. Barr, V. Dobos, L.L. Kiss, Interior structures and tidal heating in the TRAPPIST-1 planets. Astron. Astrophys. 613, A37 (2018)

J.K. Barstow, P.G.J. Irwin, Habitable worlds with JWST: transit spectroscopy of the TRAPPIST-1 system? Mon. Not. R. Astron. Soc. 461, L92-L96 (2016)

D. Bilitza, D. Altadill, V. Truhlik, V. Shubin, I. Galkin, B. Reinisch, X. Huang, International reference ionosphere 2016: from ionospheric climate to real-time weather predictions. Space Weather 15(2), 418-429 (2017)

E. Bolmont, F. Selsis, J.E. Owen, I. Ribas, S.N. Raymond, J. Leconte, M. Gillon, Water loss from terrestrial planets orbiting ultracool dwarfs: implications for the planets of TRAPPIST-1. Mon. Not. R. Astron. Soc. 464, 3728-3741 (2017a)

E. Bolmont, F. Selsis, J.E. Owen, I. Ribas, S.N. Raymond, J. Leconte, M. Gillon, Water loss from terrestrial planets orbiting ultracool dwarfs: implications for the planets of TRAPPIST-1. Mon. Not. R. Astron. Soc. 464, 3728-3741 (2017b)

E. Bolmont, B.O. Demory, S. Blanco-Cuaresma, E. Agol, S.L. Grimm, P. Auclair-Desrotour, F. Selsis, A. Leleu, Impact of tides on the transit-timing fits to the TRAPPIST-1 system. Astron. Astrophys. 635, A117 (2020)

V. Bourrier, J. de Wit, E. Bolmont, V. Stamenković, P.J. Wheatley, A.J. Burgasser, L. Delrez, B.-O. Demory, D. Ehrenreich, M. Gillon, E. Jehin, J. Leconte, S.M. Lederer, N. Lewis, A.H.M.J. Triaud, V. Van Grootel, Temporal evolution of the high-energy irradiation and water content of TRAPPIST-1 exoplanets. Astron. J. 154, 121 (2017a)

V. Bourrier, D. Ehrenreich, P.J. Wheatley, E. Bolmont, M. Gillon, J. de Wit, A.J. Burgasser, E. Jehin, D. Queloz, A.H.M.J. Triaud, Reconnaissance of the TRAPPIST-1 exoplanet system in the Lyman- $\alpha$ line. Astron. Astrophys. 599, L3 (2017b)

I.A. Boutle, N.J. Mayne, B. Drummond, J. Manners, J. Goyal, F. Hugo Lambert, D.M. Acreman, P.D. Earnshaw, Exploring the climate of proxima B with the met office unified model. Astron. Astrophys. 601, A120 (2017)

R. Brasser, A.C. Barr, V. Dobos, The tidal parameters of TRAPPIST-1b and c. Mon. Not. R. Astron. Soc. 487(1), 34-47 (2019)

A.G.A. Brown, A. Vallenari, T. Prusti, J.H.J. de Bruijne, C. Babusiaux, C.A.L. Bailer-Jones, M. Biermann, D.W. Evans, L. Eyer, F. Jansen, C. Jordi, S.A. Klioner, U. Lammers, L. Lindegren, X. Luri, F. Mignard, C. Panem, D. Pourbaix, S. Randich, P. Sartoretti, H.I. Siddiqui, C. Soubiran, F. van Leeuwen, N.A. Walton, F. Arenou, U. Bastian, M. Cropper, R. Drimmel, D. Katz, M.G. Lattanzi, J. Bakker, C. Cacciari, J. Castañeda, L. Chaoul, N. Cheek, F. De Angeli, C. Fabricius, R. Guerra, B. Holl, E. Masana, R. Messineo, N. Mowlavi, K. Nienartowicz, P. Panuzzo, J. Portell, M. Riello, G.M. Seabroke, P. Tanga, F. Thévenin, G. Gracia-Abril, G. Comoretto, M. Garcia-Reinaldos, D. Teyssier, M. Altmann, R. Andrae, M. Audard, I. Bellas-Velidis, K. Benson, J. Berthier, R. Blomme, P. Burgess, G. Busso, B. Carry, A. Cellino, G. Clementini, M. Clotet, O. Creevey, M. Davidson, J. De Ridder, L. Delchambre, A. Dell'Oro, C. Ducourant, J. Fernández-Hernández, M. Fouesneau, Y. Frémat, L. Galluccio, M. García-Torres, J. González-Núñez, J.J. González-Vidal, E. Gosset, L.P. Guy, J.L. Halbwachs, N.C. Hambly, D.L. Harrison, J. Hernández, D. Hestroffer, S.T. Hodgkin, A. Hutton, G. Jasniewicz, A. Jean-Antoine-Piccolo, S. Jordan, A.J. Korn, A. Krone-Martins, A.C. Lanzafame, T. Lebzelter, W. Löffler, M. Manteiga, P.M. Marrese, J.M. Martín-Fleitas, A. Moitinho, A. Mora, K. Muinonen, J. Osinde, E. Pancino, T. Pauwels, J.M. Petit, A. Recio-Blanco, P.J. Richards, L. Rimoldini, A.C. Robin, L.M. Sarro, C. Siopis, M. Smith, A. Sozzetti, M. Süveges, J. Torra, W. van Reeven, U. Abbas, A. Abreu Aramburu, S. Accart, C. Aerts, 
G. Altavilla, M.A. Álvarez, R. Alvarez, J. Alves, R.I. Anderson, A.H. Andrei, E. Anglada Varela, E. Antiche, T. Antoja, B. Arcay, T.L. Astraatmadja, N. Bach, S.G. Baker, L. Balaguer-Núñez, P. Balm, C. Barache, C. Barata, D. Barbato, F. Barblan, P.S. Barklem, D. Barrado, M. Barros, M.A. Barstow, S. Bartholomé Muñoz, J.L. Bassilana, U. Becciani, M. Bellazzini, A. Berihuete, S. Bertone, L. Bianchi, O. Bienaymé, S. Blanco-Cuaresma, T. Boch, C. Boeche, A. Bombrun, R. Borrachero, D. Bossini, S. Bouquillon, G. Bourda, A. Bragaglia, L. Bramante, M.A. Breddels, A. Bressan, N. Brouillet, T. Brüsemeister, E. Brugaletta, B. Bucciarelli, A. Burlacu, D. Busonero, A.G. Butkevich, R. Buzzi, E. Caffau, R. Cancelliere, G. Cannizzaro, T. Cantat-Gaudin, R. Carballo, T. Carlucci, J.M. Carrasco, L. Casamiquela, M. Castellani, A. Castro-Ginard, P. Charlot, L. Chemin, A. Chiavassa, G. Cocozza, G. Costigan, S. Cowell, F. Crifo, M. Crosta, C. Crowley, J. Cuypers, C. Dafonte, Y. Damerdji, A. Dapergolas, P. David, M. David, P. de Laverny, F. De Luise, R. De March, D. de Martino, R. de Souza, A. de Torres, J. Debosscher, E. del Pozo, M. Delbo, A. Delgado, H.E. Delgado, P. Di Matteo, S. Diakite, C. Diener, E. Distefano, C. Dolding, P. Drazinos, J. Durán, B. Edvardsson, H. Enke, K. Eriksson, P. Esquej, G. Eynard Bontemps, C. Fabre, M. Fabrizio, S. Faigler, A.J. Falcão, M. Farràs Casas, L. Federici, G. Fedorets, P. Fernique, F. Figueras, F. Filippi, K. Findeisen, A. Fonti, E. Fraile, M. Fraser, B. Frézouls, M. Gai, S. Galleti, D. Garabato, F. García-Sedano, A. Garofalo, N. Garralda, A. Gavel, P. Gavras, J. Gerssen, R. Geyer, P. Giacobbe, G. Gilmore, S. Girona, G. Giuffrida, F. Glass, M. Gomes, M. Granvik, A. Gueguen, A. Guerrier, J. Guiraud, R. Gutiérrez-Sánchez, R. Haigron, D. Hatzidimitriou, M. Hauser, M. Haywood, U. Heiter, A. Helmi, J. Heu, T. Hilger, D. Hobbs, W. Hofmann, G. Holland, H.E. Huckle, A. Hypki, V. Icardi, K. Janßen, G. Jevardat de Fombelle, P.G. Jonker, Á.L. Juhász, F. Julbe, A. Karampelas, A. Kewley, J. Klar, A. Kochoska, R. Kohley, K. Kolenberg, M. Kontizas, E. Kontizas, S.E. Koposov, G. Kordopatis, Z. Kostrzewa-Rutkowska, P. Koubsky, S. Lambert, A.F. Lanza, Y. Lasne, J.B. Lavigne, Y. Le Fustec, C. Le Poncin-Lafitte, Y. Lebreton, S. Leccia, N. Leclerc, I. Lecoeur-Taibi, H. Lenhardt, F. Leroux, S. Liao, E. Licata, H.E.P. Lindstrøm, T.A. Lister, E. Livanou, A. Lobel, M. López, S. Managau, R.G. Mann, G. Mantelet, O. Marchal, J.M. Marchant, M. Marconi, S. Marinoni, G. Marschalkó, D.J. Marshall, M. Martino, G. Marton, N. Mary, D. Massari, G. Matijevič, T. Mazeh, P.J. McMillan, S. Messina, D. Michalik, N.R. Millar, D. Molina, R. Molinaro, L. Molnár, P. Montegriffo, R. Mor, R. Morbidelli, T. Morel, D. Morris, A.F. Mulone, T. Muraveva, I. Musella, G. Nelemans, L. Nicastro, L. Noval, W. O’Mullane, C. Ordénovic, D. Ordóñez-Blanco, P. Osborne, C. Pagani, I. Pagano, F. Pailler, H. Palacin, L. Palaversa, A. Panahi, M. Pawlak, A.M. Piersimoni, F.X. Pineau, E. Plachy, G. Plum, E. Poggio, E. Poujoulet, A. Prša, L. Pulone, E. Racero, S. Ragaini, N. Rambaux, M. Ramos-Lerate, S. Regibo, C. Reylé, F. Riclet, V. Ripepi, A. Riva, A. Rivard, G. Rixon, T. Roegiers, M. Roelens, M. Romero-Gómez, N. Rowell, F. Royer, L. Ruiz-Dern, G. Sadowski, T. Sagristà Sellés, J. Sahlmann, J. Salgado, E. Salguero, N. Sanna, T. Santana-Ros, M. Sarasso, H. Savietto, M. Schultheis, E. Sciacca, M. Segol, J.C. Segovia, D. Ségransan, I.C. Shih, L. Siltala, A.F. Silva, R.L. Smart, K.W. Smith, E. Solano, F. Solitro, R. Sordo, S. Soria Nieto, J. Souchay, A. Spagna, F. Spoto, U. Stampa, I.A. Steele, H. Steidelmüller, C.A. Stephenson, H. Stoev, F.F. Suess, J. Surdej, L. Szabados, E. Szegedi-Elek, D. Tapiador, F. Taris, G. Tauran, M.B. Taylor, R. Teixeira, D. Terrett, i.P. Teyssand, W. Thuillot, A. Titarenko, F. Torra Clotet, C. Turon, A. Ulla, E. Utrilla, S. Uzzi, M. Vaillant, G. Valentini, V. Valette, A. van Elteren, E. Van Hemelryck, M. van Leeuwen, M. Vaschetto, A. Vecchiato, J. Veljanoski, Y. Viala, D. Vicente, S. Vogt, C. von Essen, H. Voss, V. Votruba, S. Voutsinas, G. Walmsley, M. Weiler, O. Wertz, T. Wevers, Ł. Wyrzykowski, A. Yoldas, M. Žerjal, H. Ziaeepour, J. Zorec, S. Zschocke, S. Zucker, C. Zurbach, T. Zwitter, (Gaia Collaboration), Gaia Data Release 2. Summary of the contents and survey properties. Astron. Astrophys. 616, A1 (2018)

A.Y. Burdanov, S.M. Lederer, M. Gillon, L. Delrez, E. Ducrot, J. de Wit, E. Jehin, A.H.M.J. Triaud, C. Lidman, L. Spitler, B.O. Demory, D. Queloz, V. Van Grootel, Ground-based follow-up observations of TRAPPIST-1 transits in the near-infrared. Mon. Not. R. Astron. Soc. 487(2), 1634-1652 (2019)

A.J. Burgasser, E.E. Mamajek, On the age of the TRAPPIST-1 system. Astrophys. J. 845, 110 (2017)

J.A. Carter, E. Agol, W.J. Chaplin, S. Basu, T.R. Bedding, L.A. Buchhave, J. Christensen-Dalsgaard, K.M. Deck, Y. Elsworth, D.C. Fabrycky, E.B. Ford, J.J. Fortney, S.J. Hale, R. Handberg, S. Hekker, M.J. Holman, D. Huber, C. Karoff, S.D. Kawaler, H. Kjeldsen, J.J. Lissauer, E.D. Lopez, M.N. Lund, M. Lundkvist, T.S. Metcalfe, A. Miglio, L.A. Rogers, D. Stello, W.J. Borucki, S. Bryson, J.L. Christiansen, W.D. Cochran, J.C. Geary, R.L. Gilliland, M.R. Haas, J. Hall, A.W. Howard, J.M. Jenkins, T. Klaus, D.G. Koch, D.W. Latham, P.J. MacQueen, D. Sasselov, J.H. Steffen, J.D. Twicken, J.N. Winn, Kepler36: a pair of planets with neighboring orbits and dissimilar densities. Science 337, 556 (2012)

G. Chabrier, I. Baraffe, Structure and evolution of low-mass stars. Astron. Astrophys. 327, 1039-1053 (1997)

J.M. Chadney, M. Galand, Y.C. Unruh, T.T. Koskinen, J. Sanz-Forcada, XUV-driven mass loss from extrasolar giant planets orbiting active stars. Icarus 250, 357-367 (2015)

S. Chapman, R. Lindzen, Atmospheric Tides. Thermal and Gravitational (1970)

H. Chen, E.T. Wolf, Z. Zhan, D.E. Horton, Habitability and spectroscopic observability of warm M-dwarf exoplanets evaluated with a 3D chemistry-climate model. Astrophys. J. 886(1), 16 (2019) 
R. Cloutier, A.H.M.J. Triaud, Prospects for detecting the Rossiter-McLaughlin effect of Earth-like planets: the test case of TRAPPIST-1b and c. Mon. Not. R. Astron. Soc. 462(4), 4018-4027 (2016)

G.A.L. Coleman, A. Leleu, Y. Alibert, W. Benz, Pebbles versus planetesimals: the case of Trappist-1. Astron. Astrophys. 631, A7 (2019)

A.C.M. Correia, J. Laskar, The four final rotation states of Venus. Nature 411, 767-770 (2001)

C. Cossou, S.N. Raymond, F. Hersant, A. Pierens, Hot super-Earths and giant planet cores from different migration histories. Astron. Astrophys. 569, A56 (2014)

J.H. Davies, Global map of solid Earth surface heat flow. Geochem. Geophys. Geosyst. 14, 4608-4622 (2013)

J.H. Davies, D.R. Davies, Earth's surface heat flux. Solid Earth 1, 5-24 (2010)

J. de Wit, H.R. Wakeford, M. Gillon, N.K. Lewis, J.A. Valenti, B.-O. Demory, A.J. Burgasser, A. Burdanov, L. Delrez, E. Jehin, S.M. Lederer, D. Queloz, A.H.M.J. Triaud, V. Van Grootel, A combined transmission spectrum of the Earth-sized exoplanets TRAPPIST-1 b and c. Nature 537, 69-72 (2016)

J. de Wit, H.R. Wakeford, N.K. Lewis, L. Delrez, M. Gillon, F. Selsis, J. Leconte, B.-O. Demory, E. Bolmont, V. Bourrier, A.J. Burgasser, S. Grimm, E. Jehin, S.M. Lederer, J.E. Owen, V. Stamenković, A.H.M.J. Triaud, Atmospheric reconnaissance of the habitable-zone Earth-sized planets orbiting TRAPPIST-1. Nat. Astron. 2, 214-219 (2018)

K.M. Deck, E. Agol, Measurement of planet masses with transit timing variations due to synodic "chopping" effects. Astrophys. J. 802(2), 116 (2015)

L. Delrez, M. Gillon, A.H.M.J. Triaud, B.-O. Demory, J. de Wit, J.G. Ingalls, E. Agol, E. Bolmont, A. Burdanov, A.J. Burgasser, S.J. Carey, E. Jehin, J. Leconte, S. Lederer, D. Queloz, F. Selsis, V. Van Grootel, Early 2017 observations of TRAPPIST-1 with Spitzer. Mon. Not. R. Astron. Soc. 475, 35773597 (2018)

Z. Dencs, Z. Regály, Water delivery to the TRAPPIST-1 planets. Mon. Not. R. Astron. Soc. 487(2), 21912199 (2019)

V. Dobos, A.C. Barr, L.L. Kiss, Tidal heating and the habitability of the TRAPPIST-1 exoplanets. Astron. Astrophys. 624, A2 (2019)

C. Dong, M. Lingam, Y. Ma, O. Cohen, Is proxima Centauri b habitable? A study of atmospheric loss. Astrophys. J. Lett. 837(2), L26 (2017)

C. Dong, M. Jin, M. Lingam, V.S. Airapetian, Y. Ma, B. van der Holst, Atmospheric escape from the TRAPPIST-1 planets and implications for habitability. Proc. Natl. Acad. Sci. 115, 260-265 (2018)

C. Dong, Z. Huang, M. Lingam, Role of planetary obliquity in regulating atmospheric escape: G-dwarf versus M-dwarf Earth-like exoplanets. Astrophys. J. Lett. 882(2), L16 (2019)

C. Dong, M. Jin, M. Lingam, Atmospheric escape from TOI-700 d: Venus versus Earth analogs. Astrophys. J. Lett. 896, L24 (2020)

C. Dorn, K. Mosegaard, S.L. Grimm, Y. Alibert, Interior characterization in multiplanetary systems: TRAPPIST-1. Astrophys. J. 865, 20 (2018)

L.A. dos Santos, V. Bourrier, D. Ehrenreich, S. Kameda, Observability of hydrogen-rich exospheres in Earthlike exoplanets. Astron. Astrophys. 622, A46 (2019)

R. Doyon, J.B. Hutchings, M. Beaulieu, L. Albert, D. Lafrenière, C. Willott, D. Touahri, N. Rowlands, M. Maszkiewicz, A.W. Fullerton, K. Volk, A.R. Martel, P. Chayer, A. Sivaramakrishnan, R. Abraham, L. Ferrarese, R. Jayawardhana, D. Johnstone, M. Meyer, J.L. Pipher, M. Sawicki, The JWST Fine Guidance Sensor (FGS) and Near-Infrared Imager and Slitless Spectrograph (NIRISS), in Society of PhotoOptical Instrumentation Engineers (SPIE) Conference Series, vol. 8442 (2012), p. 84422R

E. Ducrot, M. Sestovic, B.M. Morris, M. Gillon, A.H.M.J. Triaud, J. De Wit, D. Thimmarayappa, E. Agol, Y. Almleaky, A. Burdanov, A.J. Burgasser, L. Delrez, B.-O. Demory, E. Jehin, J. Leconte, J. McCormac, C. Murray, D. Queloz, F. Selsis, S. Thompson, V. Van Grootel, The 0.8-4.5 $\mu \mathrm{m}$ broadband transmission spectra of TRAPPIST-1 planets. Astron. J. 156, 218 (2018)

E. Ducrot, M. Gillon, L. Delrez, E. Agol, P. Rimmer, M. Turbet, M.N. Günther, B.-O. Demory, A.H.M.J. Triaud, E. Bolmont, A. Burgasser, S.J. Carey, J.G. Ingalls, E. Jehin, J. Leconte, S.M. Lederer, D. Queloz, S.N. Raymond, F. Selsis, V. Van Grootel, J. de Wit, TRAPPIST-1: Global Results of the Spitzer Exploration Science Program Red Worlds. (2020). ArXiv e-prints, arXiv:2006.13826

N.V. Erkaev, Y.N. Kulikov, H. Lammer, F. Selsis, D. Langmayr, G.F. Jaritz, H.K. Biernat, Roche lobe effects on the atmospheric loss from "Hot Jupiters". Astron. Astrophys. 472, 329-334 (2007)

T.J. Fauchez, M. Turbet, G.L. Villanueva, E.T. Wolf, G. Arney, R.K. Kopparapu, A. Lincowski, A. Mandell, J. de Wit, D. Pidhorodetska, S.D. Domagal-Goldman, K.B. Stevenson, Impact of clouds and hazes on the simulated JWST transmission spectra of habitable zone planets in the TRAPPIST-1 system. Astrophys. J. 887(2), 194 (2019)

T. Fauchez, G. Villanueva, E.W. Schwieterman, M. Turbet, G. Arney, D. Pidhorodetska, R.K. Kopparapu, A. Mandell, S.D. Domagal-Goldman, Sensitive probing of exoplanetary oxygen via mid-infrared collisional absorption. Nat. Astron. 4, 372-376 (2020a) 
T.J. Fauchez, M. Turbet, E.T. Wolf, I. Boutle, M.J. Way, A.D. Del Genio, N.J. Mayne, K. Tsigaridis, R.K. Kopparapu, J. Yang, F. Forget, A. Mand ell, S.D. Domagal Goldman, TRAPPIST-1 Habitable Atmosphere Intercomparison (THAI): motivations and protocol version 1.0. Geosci. Model Dev. 13(2), 707716 (2020b)

J.A. Fennelly, D.G. Torr, Photoionization and photoabsorption cross sections of $\mathrm{O}, \mathrm{N}_{2} \mathrm{O}_{2}$, and $\mathrm{N}$ for aeronomic calculations. At. Data Nucl. Data Tables 51, 321 (1992)

P. Ferruit, S. Birkmann, T. Böker, M. Sirianni, G. Giardino, G. de Marchi, C. Alves de Oliveira, B. Dorner, Observing transiting exoplanets with JWST/NIRSpec, in Society of Photo-Optical Instrumentation Engineers (SPIE) Conference Series, vol. 9143, (2014), p. 91430A

J.C. Filippazzo, E.L. Rice, J. Faherty, K.L. Cruz, M.M. Van Gordon, D.L. Looper, Fundamental parameters and spectral energy distributions of young and field age objects with masses spanning the stellar to planetary regime. Astrophys. J. 810(2), 158 (2015)

D.P. Fleming, R. Barnes, R. Luger, J.T. VanderPlas, On the XUV luminosity evolution of TRAPPIST-1. Astrophys. J. 891(2), 155 (2020)

R. Follert, R.J. Dorn, E. Oliva, J.L. Lizon, A. Hatzes, N. Piskunov, A. Reiners, U. Seemann, E. Stempels, U. Heiter, T. Marquart, M. Lockhart, G. Anglada-Escude, T. Löwinger, D. Baade, J. Grunhut, P. Bristow, B. Klein, Y. Jung, D.J. Ives, F. Kerber, E. Pozna, J. Paufique, H.U. Kaeufl, L. Origlia, E. Valenti, D. Gojak, M. Hilker, L. Pasquini, A. Smette, J. Smoker, CRIRES+: a cross-dispersed high-resolution infrared spectrograph for the ESO VLT, in Society of Photo-Optical Instrumentation Engineers (SPIE) Conference Series, vol. 9147, (2014), p. 914719

F. Fraschetti, J.J. Drake, J.D. Alvarado-Gómez, S.P. Moschou, C. Garraffo, O. Cohen, Stellar energetic particles in the magnetically turbulent habitable zones of TRAPPIST-1-like planetary systems. Astrophys. J. 874, 21 (2019)

P. Gao, R. Hu, T.D. Robinson, C. Li, Y.L. Yung, Stability of CO2 atmospheres on desiccated M dwarf exoplanets. Astrophys. J. 806(2), 249 (2015)

C. Garraffo, J.J. Drake, O. Cohen, J.D. Alvarado-Gómez, S.P. Moschou, The threatening magnetic and plasma environment of the TRAPPIST-1 planets. Astrophys. J. Lett. 843, L33 (2017)

M. Gillon, E. Jehin, P. Magain, V. Chantry, D. Hutsemékers, J. Manfroid, D. Queloz, S. Udry, TRAPPIST: a robotic telescope dedicated to the study of planetary systems, in European Physical Journal Web of Conferences. European Physical Journal Web of Conferences, vol. 11 (2011), p. 06002

M. Gillon, E. Jehin, A. Fumel, P. Magain, D. Queloz, TRAPPIST-UCDTS: a prototype search for habitable planets transiting ultra-cool stars, in European Physical Journal Web of Conferences. European Physical Journal Web of Conferences, vol. 47 (2013), p. 03001

M. Gillon, E. Jehin, S.M. Lederer, L. Delrez, J. de Wit, A. Burdanov, V. Van Grootel, A.J. Burgasser, A.H.M.J. Triaud, C. Opitom, B.-O. Demory, D.K. Sahu, D. Bardalez Gagliuffi, P. Magain, D. Queloz, Temperate Earth-sized planets transiting a nearby ultracool dwarf star. Nature 533, 221-224 (2016)

M. Gillon, A.H.M.J. Triaud, B.-O. Demory, E. Jehin, E. Agol, K.M. Deck, S.M. Lederer, J. de Wit, A. Burdanov, J.G. Ingalls, E. Bolmont, J. Leconte, S.N. Raymond, F. Selsis, M. Turbet, K. Barkaoui, A. Burgasser, M.R. Burleigh, S.J. Carey, A. Chaushev, C.M. Copperwheat, L. Delrez, C.S. Fernandes, D.L. Holdsworth, E.J. Kotze, V. Van Grootel, Y. Almleaky, Z. Benkhaldoun, P. Magain, D. Queloz, Seven temperate terrestrial planets around the nearby ultracool dwarf star TRAPPIST-1. Nature 542, 456-460 (2017)

M. Gillon, V. Meadows, E. Agol, A.J. Burgasser, D. Deming, R. Doyon, J. Fortney, L. Kreidberg, J. Owen, F. Selsis, J. de Wit, J. Lustig-Yaeger, B.V. Rackham, The TRAPPIST-1 JWST Community Initiative. (2020). ArXiv e-prints, arXiv:2002.04798

C. Goldblatt, A.J. Watson, The runaway greenhouse: implications for future climate change, geoengineering and planetary atmospheres. Philos. Trans. R. Soc. Lond. Ser. A, Math. Phys. Sci. 370, 4197-4216 (2012)

C. Goldblatt, T.D. Robinson, K.J. Zahnle, D. Crisp, Low simulated radiation limit for runaway greenhouse climates. Nat. Geosci. 6, 661-667 (2013)

P. Goldreich, S. Peale, Spin-orbit coupling in the solar system. Astrophys. J. 71, 425 (1966)

E.C. Gonzales, J.K. Faherty, J. Gagné, J. Teske, A. McWilliam, K. Cruz, A reanalysis of the fundamental parameters and age of TRAPPIST-1. Astrophys. J. 886(2), 131 (2019)

I.E. Gordon, L.S. Rothman, C. Hill, R.V. Kochanov, Y. Tan, P.F. Bernath, M. Birk, V. Boudon, A. Campargue, K.V. Chance, B.J. Drouin, J.M. Flaud, R.R. Gamache, J.T. Hodges, D. Jacquemart, V.I. Perevalov, A. Perrin, K.P. Shine, M.A.H. Smith, J. Tennyson, G.C. Toon, H. Tran, V.G. Tyuterev, A. Barbe, A.G. Császár, V.M. Devi, T. Furtenbacher, J.J. Harrison, J.M. Hartmann, A. Jolly, T.J. Johnson, T. Karman, I. Kleiner, A.A. Kyuberis, J. Loos, O.M. Lyulin, S.T. Massie, S.N. Mikhailenko, N. Moazzen-Ahmadi, H.S.P. Müller, O.V. Naumenko, A.V. Nikitin, O.L. Polyansky, M. Rey, M. Rotger, S.W. Sharpe, K. Sung, E. Starikova, S.A. Tashkun, J.V.E.Auwera, G. Wagner, J. Wilzewski, P. Wcisło, S. Yu, E.J. Zak, The HITRAN2016 molecular spectroscopic database. J. Quant. Spectrosc. Radiat. Transf. 203, 3-69 (2017) 
S.L. Grimm, B.-O. Demory, M. Gillon, C. Dorn, E. Agol, A. Burdanov, L. Delrez, M. Sestovic, A.H.M.J. Triaud, M. Turbet, É. Bolmont, A. Caldas, J. de Wit, E. Jehin, J. Leconte, S.N. Raymond, V. Van Grootel, A.J. Burgasser, S. Carey, D. Fabrycky, K. Heng, D.M. Hernandez, J.G. Ingalls, S. Lederer, F. Selsis, D. Queloz, The nature of the TRAPPIST-1 exoplanets. Astron. Astrophys. 613, A68 (2018)

K. Hamano, Y. Abe, H. Genda, Emergence of two types of terrestrial planet on solidification of magma ocean. Nature 497, 607-610 (2013)

H.C.F.C. Hay, I. Matsuyama, Tides between the TRAPPIST-1 planets. Astrophys. J. 875(1), 22 (2019)

A.N. Heays, A.D. Bosman, E.F. van Dishoeck, Photodissociation and photoionisation of atoms and molecules of astrophysical interest. Astron. Astrophys. 602, A105 (2017)

É.M. Hébrard, J.F. Donati, X. Delfosse, J. Morin, C. Moutou, I. Boisse, Modelling the RV jitter of early-M dwarfs using tomographic imaging. Mon. Not. R. Astron. Soc. 461(2), 1465-1497 (2016)

N.R. Hinkel, F.X. Timmes, P.A. Young, M.D. Pagano, M.C. Turnbull, Stellar abundances in the solar neighborhood: the Hypatia Catalog. Astron. J. 148(3), 54 (2014)

T. Hirano, E. Gaidos, J.N. Winn, F. Dai, A. Fukui, M. Kuzuhara, T. Kotani, M. Tamura, M. Hjorth, S. Albrecht, D. Huber, E. Bolmont, H. Harakawa, K. Hodapp, M. Ishizuka, S. Jacobson, M. Konishi, T. Kudo, T. Kurokawa, J. Nishikawa, M. Omiya, T. Serizawa, A. Ueda, L.M. Weiss, Evidence for spin-orbit alignment in the TRAPPIST-1 system. Astrophys. J. Lett. 890(2), L27 (2020)

M.J. Holman, N.W. Murray, The use of transit timing to detect terrestrial-mass extrasolar planets. Science 307, 1288-1291 (2005)

M.J. Holman, D.C. Fabrycky, D. Ragozzine, E.B. Ford, J.H. Steffen, W.F. Welsh, J.J. Lissauer, D.W. Latham, G.W. Marcy, L.M. Walkowicz, N.M. Batalha, J.M. Jenkins, J.F. Rowe, W.D. Cochran, F. Fressin, G. Torres, L.A. Buchhave, D.D. Sasselov, W.J. Borucki, D.G. Koch, G. Basri, T.M. Brown, D.A. Caldwell, D. Charbonneau, E.W. Dunham, T.N. Gautier, J.C. Geary, R.L. Gilliland, M.R. Haas, S.B. Howell, D.R. Ciardi, M. Endl, D. Fischer, G. Fürész, J.D. Hartman, H. Isaacson, J.A. Johnson, P.J. MacQueen, A.V. Moorhead, R.C. Morehead, J.A. Orosz, Kepler-9: a system of multiple planets transiting a Sun-like star, confirmed by timing variations. Science 330(6000), 51 (2010)

Y. Hori, M. Ogihara, Do the TRAPPIST-1 planets have hydrogen-rich atmospheres? Astrophys. J. 889(2), 77 (2020)

S.B. Howell, C. Sobeck, M. Haas, M. Still, T. Barclay, F. Mullally, J. Troeltzsch, S. Aigrain, S.T. Bryson, D. Caldwell, W.J. Chaplin, W.D. Cochran, D. Huber, G.W. Marcy, A. Miglio, J.R. Najita, M. Smith, J.D. Twicken, J.J. Fortney, The K2 mission: characterization and early results. Publ. Astron. Soc. Pac. 126, 398 (2014)

R. Hu, L. Peterson, E.T. Wolf, $\mathrm{O}_{2}$ - and $\mathrm{CO}$-rich atmospheres for potentially habitable environments on TRAPPIST-1 planets. Astrophys. J. 888(2), 122 (2020)

A.P. Ingersoll, The runaway greenhouse: a history of water on Venus. J. Atmos. Sci. 26, 1191-1198 (1969)

A.P. Ingersoll, A.R. Dobrovolskis, Venus' rotation and atmospheric tides. Nature 275(5675), 37-38 (1978)

A. Izidoro, M. Ogihara, S.N. Raymond, A. Morbidelli, A. Pierens, B. Bitsch, C. Cossou, F. Hersant, Breaking the chains: hot super-Earth systems from migration and disruption of compact resonant chains. Mon. Not. R. Astron. Soc. 470(2), 1750-1770 (2017)

C.P. Johnstone, Hydrodynamic escape of water vapor atmospheres near very active stars. Astrophys. J. 890(1), $79(2020)$

C.P. Johnstone, M. Güdel, H. Lammer, K.G. Kislyakova, Upper atmospheres of terrestrial planets: carbon dioxide cooling and the Earth's thermospheric evolution. Astron. Astrophys. 617, A107 (2018)

M.M. Joshi, R.M. Haberle, R.T. Reynolds, Simulations of the atmospheres of synchronously rotating terrestrial planets orbiting M dwarfs: conditions for atmospheric collapse and the implications for habitability. Icarus 129(2), 450-465 (1997)

S. Kameda, A. Tavrov, G. Murakami, K. Enya, M. Ikoma, N. Narita, H. Fujiwara, N. Terada, O. Korablev, M. Sachkov, Observability of oxygen exosphere of an Earth-like exoplanet around a low temperature star, in EPSC-DPS Joint Meeting 2019, vol. 2019 (2019), EPSC-DPS2019-1031

S.R. Kane, The impact of stellar distances on habitable zone planets. Astrophys. J. Lett. 861, L21 (2018)

J.F. Kasting, Stability of ammonia in the primitive terrestrial atmosphere. J. Geophys. Res. 87(C4), 30913098 (1982)

J.F. Kasting, Runaway and moist greenhouse atmospheres and the evolution of Earth and Venus. Icarus 74, 472-494 (1988)

H. Keller-Rudek, G.K. Moortgat, R. Sander, R. Sörensen, The MPI-Mainz UV/VIS spectral atlas of gaseous molecules of atmospheric interest. Earth Syst. Sci. Data 5(2), 365-373 (2013)

S. Kendrew, S. Scheithauer, P. Bouchet, J. Amiaux, R. Azzollini, J. Bouwman, C.H. Chen, D. Dubreuil, S. Fischer, A. Glasse, T.P. Greene, P.O. Lagage, F. Lahuis, S. Ronayette, D. Wright, G.S. Wright, The midinfrared instrument for the James Webb Space Telescope, IV: the low-resolution spectrometer. Publ. Astron. Soc. Pac. 127(953), 623 (2015) 
K.G. Kislyakova, L. Noack, C.P. Johnstone, V.V. Zaitsev, L. Fossati, H. Lammer, M.L. Khodachenko, P. Odert, M. Guedel, Magma oceans and enhanced volcanism on TRAPPIST-1 planets due to induction heating. Nat. Astron. 1, 878-885 (2017)

B. Klein, J.F. Donati, Simulating radial velocity observations of trappist-1 with SPIRou. Mon. Not. R. Astron. Soc. 488(4), 5114-5126 (2019)

D.D.B. Koll, D.S. Abbot, Temperature structure and atmospheric circulation of dry tidally locked rocky exoplanets. Astrophys. J. 825(2), 99 (2016)

D.D.B. Koll, M. Malik, M. Mansfield, E.M.R. Kempton, E. Kite, D. Abbot, J.L. Bean, Identifying candidate atmospheres on rocky M dwarf planets via eclipse photometry. Astrophys. J. 886(2), 140 (2019)

T.D. Komacek, T.J. Fauchez, E.T. Wolf, D.S. Abbot, Clouds will likely prevent the detection of water vapor in JWST transmission spectra of terrestrial exoplanets. Astrophys. J. Lett. 888(2), L20 (2020)

R.K. Kopparapu, R. Ramirez, J.F. Kasting, V. Eymet, T.D. Robinson, S. Mahadevan, R.C. Terrien, S. Domagal-Goldman, V. Meadows, R. Deshpande, Erratum: Habitable zones around main-sequence stars: new estimates (Astrophysical journal (2013) 765 (131)). Astrophys. J. 770, 82 (2013a)

R.K. Kopparapu, R. Ramirez, J.F. Kasting, V. Eymet, T.D. Robinson, S. Mahadevan, R.C. Terrien, S. Domagal-Goldman, V. Meadows, R. Deshpande, Habitable zones around main-sequence stars: new estimates. Astrophys. J. 765, 131 (2013b)

R.K. Kopparapu, E.T. Wolf, J. Haqq-Misra, J. Yang, J.F. Kasting, V. Meadows, R. Terrien, S. Mahadevan, The inner edge of the habitable zone for synchronously rotating planets around low-mass stars using general circulation models. Astrophys. J. 819, 84 (2016)

T. Kotani, M. Tamura, J. Nishikawa, A. Ueda, M. Kuzuhara, M. Omiya, J. Hashimoto, M. Ishizuka, T. Hirano, H. Suto, T. Kurokawa, T. Kokubo, T. Mori, Y. Tanaka, K. Kashiwagi, M. Konishi, T. Kudo, B. Sato, S. Jacobson, K.W. Hodapp, D.B. Hall, W. Aoki, T. Usuda, S. Nishiyama, T. Nakajima, Y. Ikeda, T. Yamamuro, J.-I. Morino, H. Baba, K. Hosokawa, H. Ishikawa, N. Narita, E. Kokubo, Y. Hayano, H. Izumiura, E. Kambe, N. Kusakabe, J. Kwon, M. Ikoma, Y. Hori, H. Genda, A. Fukui, Y. Fujii, H. Kawahara, G. Olivier, N. Jovanovic, H. Harakawa, M. Hayashi, M. Hidai, M. Machida, T. Matsuo, T. Nagata, M. Ogihara, H. Takami, N. Takato, H. Terada, D. Oh, The infrared Doppler (IRD) instrument for the Subaru telescope: instrument description and commissioning results, in Proceedings of the SPIE. Society of Photo-Optical Instrumentation Engineers (SPIE) Conference Series, vol. 10702 (2018), p. 1070211

Q. Kral, M.C. Wyatt, A.H.M.J. Triaud, S. Marino, P. Thébault, O. Shorttle, Cometary impactors on the TRAPPIST-1 planets can destroy all planetary atmospheres and rebuild secondary atmospheres on planets f, g, and h. Mon. Not. R. Astron. Soc. 479(2), 2649-2672 (2018)

V.A. Krasnopolsky, A photochemical model of Titan's atmosphere and ionosphere. Icarus 201, 226-256 (2009)

V.A. Krasnopolsky, Chemical composition of Titan atmosphere and ionosphere: observations and the photochemical model. Icarus 236, 83-91 (2014)

L. Kreidberg, D.D.B. Koll, C. Morley, R. Hu, L. Schaefer, D. Deming, K.B. Stevenson, J. Dittmann, A. Vanderburg, D. Berardo, X. Guo, K. Stassun, I. Crossfield, D. Charbonneau, D.W. Latham, A. Loeb, G. Ricker, S. Seager, R. Vanderspek, Absence of a thick atmosphere on the terrestrial exoplanet LHS 3844b. Nature 573(7772), 87-90 (2019)

J. Krissansen-Totton, R. Garland, P. Irwin, D.C. Catling, Detectability of biosignatures in anoxic atmospheres with the James Webb Space Telescope: a TRAPPIST-1e case study. Astron. J. 156(3), 114 (2018)

H. Lammer, F. Selsis, I. Ribas, E.F. Guinan, S.J. Bauer, W.W. Weiss, Atmospheric loss of exoplanets resulting from stellar X-ray and extreme-ultraviolet heating. Astrophys. J. Lett. 598, L121-L124 (2003)

J. Leconte, F. Forget, B. Charnay, R. Wordsworth, F. Selsis, E. Millour, A. Spiga, 3D climate modeling of close-in land planets: circulation patterns, climate moist bistability, and habitability. Astron. Astrophys. 554, A69 (2013)

J. Leconte, H. Wu, K. Menou, N. Murray, Asynchronous rotation of Earth-mass planets in the habitable zone of lower-mass stars. Science 347, 632-635 (2015)

M.-C. Liang, A.N. Heays, B.R. Lewis, S.T. Gibson, Y.L. Yung, Source of nitrogen isotope anomaly in HCN in the atmosphere of Titan. Astrophys. J. Lett. 664, L115-L118 (2007)

H.I.M. Lichtenegger, H. Lammer, J.M. Grießmeier, Aeronomical evidence for higher $\mathrm{CO}_{2}$ levels during Earth's Hadean epoch. Icarus 210(1), 1-7 (2010)

A.P. Lincowski, V.S. Meadows, D. Crisp, T.D. Robinson, R. Luger, J. Lustig-Yaeger, G.N. Arney, Evolved climates and observational discriminants for the TRAPPIST-1 planetary system. Astrophys. J. 867(1), 76 (2018)

A.P. Lincowski, J. Lustig-Yaeger, V.S. Meadows, Observing isotopologue bands in terrestrial exoplanet atmospheres with the James Webb Space Telescope: implications for identifying past atmospheric and ocean loss. Astron. J. 158(1), 26 (2019) 
L. Lindegren, J. Hernández, A. Bombrun, S. Klioner, U. Bastian, M. Ramos-Lerate, A. de Torres, H. Steidelmüller, C. Stephenson, D. Hobbs, U. Lammers, M. Biermann, R. Geyer, T. Hilger, D. Michalik, U. Stampa, P.J. McMillan, J. Castañeda, M. Clotet, G. Comoretto, M. Davidson, C. Fabricius, G. Gracia, N.C. Hambly, A. Hutton, A. Mora, J. Portell, F. van Leeuwen, U. Abbas, A. Abreu, M. Altmann, A. Andrei, E. Anglada, L. Balaguer-Núñez, C. Barache, U. Becciani, S. Bertone, L. Bianchi, S. Bouquillon, G. Bourda, T. Brüsemeister, B. Bucciarelli, D. Busonero, R. Buzzi, R. Cancelliere, T. Carlucci, P. Charlot, N. Cheek, M. Crosta, C. Crowley, J. de Bruijne, F. de Felice, R. Drimmel, P. Esquej, A. Fienga, E. Fraile, M. Gai, N. Garralda, J.J. González-Vidal, R. Guerra, M. Hauser, W. Hofmann, B. Holl, S. Jordan, M.G. Lattanzi, H. Lenhardt, S. Liao, E. Licata, T. Lister, W. Löffler, J. Marchant, J.M. Martin-Fleitas, R. Messineo, F. Mignard, R. Morbidelli, E. Poggio, A. Riva, N. Rowell, E. Salguero, M. Sarasso, E. Sciacca, H. Siddiqui, R.L. Smart, A. Spagna, I. Steele, F. Taris, J. Torra, A. van Elteren, W. van Reeven, A. Vecchiato, Gaia Data Release 2. The astrometric solution. Astron. Astrophys. 616, A2 (2018)

J.L. Linsky, J. Fontenla, K. France, The intrinsic extreme ultraviolet fluxes of F5 V TO M5 V stars. Astrophys. J. 780, 61 (2014)

K. Lodders, H. Palme, H.P. Gail, Abundances of the elements in the solar system, in Landolt-Börnstein, vol. 4B (2009), p. 712

E.D. Lopez, J.J. Fortney, N. Miller, How thermal evolution and mass-loss sculpt populations of super-Earths and sub-Neptunes: application to the Kepler-11 system and beyond. Astrophys. J. 761(1), 59 (2012)

R.D. Lorenz, C.P. McKay, J.I. Lunine, Photochemically-induced collapse of Titan's atmosphere. Science 275, 642-644 (1997)

R. Luger, R. Barnes, Extreme water loss and abiotic O2Buildup on planets throughout the habitable zones of M dwarfs. Astrobiology 15, 119-143 (2015)

R. Luger, J. Lustig-Yaeger, E. Agol, Planet-planet occultations in TRAPPIST-1 and other exoplanet systems. Astrophys. J. 851, 94 (2017a)

R. Luger, M. Sestovic, E. Kruse, S.L. Grimm, B.-O. Demory, E. Agol, E. Bolmont, D. Fabrycky, C.S. Fernandes, V. Van Grootel, A. Burgasser, M. Gillon, J.G. Ingalls, E. Jehin, S.N. Raymond, F. Selsis, A.H.M.J. Triaud, T. Barclay, G. Barentsen, S.B. Howell, L. Delrez, J. de Wit, D. Foreman-Mackey, D.L. Holdsworth, J. Leconte, S. Lederer, M. Turbet, Y. Almleaky, Z. Benkhaldoun, P. Magain, B.M. Morris, K. Heng, D. Queloz, A seven-planet resonant chain in TRAPPIST-1. Nat. Astron. 1, 0129 (2017b)

J. Lustig-Yaeger, V.S. Meadows, A.P. Lincowski, The detectability and characterization of the TRAPPIST-1 exoplanet atmospheres with JWST. Astron. J. 158(1), 27 (2019)

M.G. MacDonald, R.I. Dawson, Three pathways for observed resonant chains. Astron. J. 156(5), 228 (2018)

V.V. Makarov, Conditions of passage and entrapment of terrestrial planets in spin-orbit resonances. Astrophys. J. 752(1), 73 (2012)

V.V. Makarov, C.T. Berghea, M. Efroimsky, Spin-orbital tidal dynamics and tidal heating in the TRAPPIST-1 multiplanet system. Astrophys. J. 857(2), 142 (2018)

M. Malik, E.M.R. Kempton, D.D.B. Koll, M. Mansfield, J.L. Bean, E. Kite, Analyzing atmospheric temperature profiles and spectra of M dwarf rocky planets. Astrophys. J. 886(2), 142 (2019)

S. Marino, M.C. Wyatt, G.M. Kennedy, M. Kama, L. Matrà, A.H.M.J. Triaud, T. Henning, Searching for a dusty cometary belt around TRAPPIST-1 with ALMA. Mon. Not. R. Astron. Soc. 492(4), 6067-6073 (2020)

F.S. Masset, A. Morbidelli, A. Crida, J. Ferreira, Disk surface density transitions as protoplanet traps. Astrophys. J. 642(1), 478-487 (2006)

N.J. Mayne, I. Baraffe, D.M. Acreman, C. Smith, N. Wood, D.S. Amundsen, J. Thuburn, D.R. Jackson, Using the UM dynamical cores to reproduce idealised 3-D flows. Geosci. Model Dev. 7(6), 3059-3087 (2014)

M. Mayor, D. Queloz, A Jupiter-mass companion to a solar-type star. Nature 378, 355-359 (1995)

D.B. McLaughlin, Some results of a spectrographic study of the Algol system. Astrophys. J. 60, 22-31 (1924)

M. Meftah, L. Damé, D. Bolsée, A. Hauchecorne, N. Pereira, D. Sluse, G. Cessateur, A. Irbah, J. Bureau, M. Weber, K. Bramstedt, T. Hilbig, R. Thiéblemont, M. Marchand, F. Lefèvre, A. Sarkissian, S. Bekki, SOLAR-ISS: a new reference spectrum based on SOLAR/SOLSPEC observations. Astron. Astrophys. 611, A1 (2018)

S.E. Moran, S.M. Hörst, N.E. Batalha, N.K. Lewis, H.R. Wakeford, Limits on clouds and hazes for the TRAPPIST-1 planets. Astron. J. 156, 252 (2018)

C.V. Morley, L. Kreidberg, Z. Rustamkulov, T. Robinson, J.J. Fortney, Observing the atmospheres of known temperate Earth-sized planets with JWST. Astrophys. J. 850, 121 (2017)

B.M. Morris, E. Agol, J.R.A. Davenport, L. Hebb, S.L. Hawley, Possible bright starspots on TRAPPIST-1. Astrophys. J. 857(1), 39 (2018a)

B.M. Morris, E. Agol, L. Hebb, S.L. Hawley, Robust transiting exoplanet radii in the presence of starspots from ingress and egress durations. Astron. J. 156(3), 91 (2018b) 
B.M. Morris, E. Agol, L. Hebb, S.L. Hawley, M. Gillon, E. Ducrot, L. Delrez, J. Ingalls, B.-O. Demory, Non-detection of contamination by stellar activity in the spitzer transit light curves of TRAPPIST-1. Astrophys. J. 863(2), L32 (2018c)

R.A. Murray-Clay, E.I. Chiang, N. Murray, Atmospheric escape from hot Jupiters. Astrophys. J. 693(1), 23-42 (2009)

M. Ogihara, S. Ida, N-body simulations of planetary accretion around M dwarf stars. Astrophys. J. 699(1), 824-838 (2009)

J.T. O’Malley-James, L. Kaltenegger, UV surface habitability of the TRAPPIST-1 system. Mon. Not. R. Astron. Soc. 469, L26-L30 (2017)

C.W. Ormel, B. Liu, D. Schoonenberg, Formation of TRAPPIST-1 and other compact systems. Astron. Astrophys. 604, A1 (2017)

J.E. Owen, S. Mohanty, Habitability of terrestrial-mass planets in the HZ of M dwarfs-I. H/He-dominated atmospheres. Mon. Not. R. Astron. Soc. 459, 4088-4108 (2016)

J.C.B. Papaloizou, E. Szuszkiewicz, C. Terquem, The TRAPPIST-1 system: orbital evolution, tidal dissipation, formation and habitability. Mon. Not. R. Astron. Soc. 476(4), 5032-5056 (2018)

S. Peacock, T. Barman, E.L. Shkolnik, P.H. Hauschildt, E. Baron, Predicting the extreme ultraviolet radiation environment of exoplanets around low-mass stars: the TRAPPIST-1 system. Astrophys. J. 871, 235 (2019)

D. Pidhorodetska, T. Fauchez, G. Villanueva, S. Domagal-Goldman, Detectability of molecular signatures on TRAPPIST-1e through transmission spectroscopy simulated for future space-based observatories (2020). ArXiv e-prints, arXiv:2001.01338

R.T. Pierrehumbert, Principles of Planetary Climate (2010)

R. Pierrehumbert, E. Gaidos, Hydrogen greenhouse planets beyond the habitable zone. Astrophys. J. Lett. 734, L13 (2011)

B. Quarles, E.V. Quintana, E. Lopez, J.E. Schlieder, T. Barclay, Plausible compositions of the seven TRAPPIST-1 planets using long-term dynamical simulations. Astrophys. J. Lett. 842(1), L5 (2017)

A. Quirrenbach, P.J. Amado, J.A. Caballero, R. Mundt, A. Reiners, I. Ribas, W. Seifert, M. Abril, J. Aceituno, F.J. Alonso-Floriano, M. Ammler-von Eiff, R. Antona Jiménez, H. Anwand-Heerwart, M. Azzaro, F. Bauer, D. Barrado, S. Becerril, V.J.S. Béjar, D. Benítez, Z.M. Berdiñas, M.C. Cárdenas, E. Casal, A. Claret, J. Colomé, M. Cortés-Contreras, S. Czesla, M. Doellinger, S. Dreizler, C. Feiz, M. Fernández, D. Galadí, M.C. Gálvez-Ortiz, A. García-Piquer, M.L. García-Vargas, R. Garrido, L. Gesa, V. Gómez Galera, E. González Álvarez, J.I. González Hernández, U. Grözinger, J. Guàrdia, E.W. Guenther, E. de Guindos, J. Gutiérrez-Soto, H.J. Hagen, A.P. Hatzes, P.H. Hauschildt, J. Helmling, T. Henning, D. Hermann, L. Hernández Castaño, E. Herrero, D. Hidalgo, G. Holgado, A. Huber, K.F. Huber, S. Jeffers, V. Joergens, E. de Juan, M. Kehr, R. Klein, M. Kürster, A. Lamert, S. Lalitha, W. Laun, U. Lemke, R. Lenzen, M. López del Fresno, B. López Martí, J. López-Santiago, U. Mall, H. Mandel, E.L. Martín, S. Martín-Ruiz, H. Martínez-Rodríguez, C.J. Marvin, R.J. Mathar, E. Mirabet, D. Montes, R. Morales Muñoz, A. Moya, V. Naranjo, A. Ofir, R. Oreiro, E. Pallé, J. Panduro, V.M. Passegger, A. Pérez-Calpena, D. Pérez Medialdea, M. Perger, M. Pluto, A. Ramón, R. Rebolo, P. Redondo, S. Reffert, S. Reinhardt, P. Rhode, H.W. Rix, F. Rodler, E. Rodríguez, C. Rodríguez-López, E. Rodríguez-Pérez, R.R. Rohloff, A. Rosich, E. Sánchez-Blanco, M.A. Sánchez Carrasco, J. Sanz-Forcada, L.F. Sarmiento, S. Schäfer, J. Schiller, C. Schmidt, J.H.M.M. Schmitt, E. Solano, O. Stahl, C. Storz, J. Stürmer, J.C. Suárez, R.G. Ulbrich, G. Veredas, K. Wagner, J. Winkler, M.R. Zapatero Osorio, M. Zechmeister, F.J. Abellán de Paco, G. Anglada-Escudé, C. del Burgo, A. Klutsch, J.L. Lizon, M. López-Morales, J.C. Morales, M.A.C. Perryman, S.M. Tulloch, W. Xu, CARMENES instrument overview, in Society of Photo-Optical Instrumentation Engineers (SPIE) Conference Series, vol. 9147 (2014), p. 91471F

B.V. Rackham, D. Apai, M.S. Giampapa, The transit light source effect: false spectral features and incorrect densities for M-dwarf transiting planets. Astrophys. J. 853, 122 (2018)

R.M. Ramirez, L. Kaltenegger, The habitable zones of pre-main-sequence stars. Astrophys. J. Lett. 797, L25 (2014)

R.M. Ramirez, L. Kaltenegger, A volcanic hydrogen habitable zone. Astrophys. J. Lett. 837, L4 (2017)

S. Ranjan, R. Wordsworth, D.D. Sasselov, The surface UV environment on planets orbiting M dwarfs: implications for prebiotic chemistry and the need for experimental follow-up. Astrophys. J. 843, 110 (2017)

A. Reiners, M. Zechmeister, J.A. Caballero, I. Ribas, J.C. Morales, S.V. Jeffers, P. Schöfer, L. Tal-Or, A. Quirrenbach, P.J. Amado, A. Kaminski, W. Seifert, M. Abril, J. Aceituno, F.J. Alonso-Floriano, M. Ammler-von Eiff, R. Antona, G. Anglada-Escudé, H. Anwand-Heerwart, B. Arroyo-Torres, M. Azzaro, D. Baroch, D. Barrado, F.F. Bauer, S. Becerril, V.J.S. Béjar, D. Benítez, Z.M. Berdinas, G. Bergond, M. Blümcke, M. Brinkmöller, C. del Burgo, J. Cano, M.C. Cárdenas Vázquez, E. Casal, C. Cifuentes, A. Claret, J. Colomé, M. Cortés-Contreras, S. Czesla, E. Díez-Alonso, S. Dreizler, C. Feiz, M. Fernández, I.M. Ferro, B. Fuhrmeister, D. Galadí-Enríquez, A. Garcia-Piquer, M.L. García Vargas, L. Gesa, V. Gómez Galera, J.I. González Hernández, R. González-Peinado, U. Grözinger, S. Grohnert, J. Guàrdia, 
E.W. Guenther, A. Guijarro, E. de Guindos, J. Gutiérrez-Soto, H.J. Hagen, A.P. Hatzes, P.H. Hauschildt, R.P. Hedrosa, J. Helmling, T. Henning, I. Hermelo, R. Hernández Arabí, L. Hernández Castaño, F. Hernández Hernando, E. Herrero, A. Huber, P. Huke, E.N. Johnson, E. de Juan, M. Kim, R. Klein, J. Klüter, A. Klutsch, M. Kürster, M. Lafarga, A. Lamert, M. Lampón, L.M. Lara, W. Laun, U. Lemke, R. Lenzen, R. Launhardt, M. López del Fresno, J. López-González, M. López-Puertas, J.F. López Salas, J. López-Santiago, R. Luque, H. Magán Madinabeitia, U. Mall, L. Mancini, H. el Mand, E. Marfil, J.A. Marín Molina, D. Maroto Fernández, E.L. Martín, S. Martín-Ruiz, C.J. Marvin, R.J. Mathar, E. Mirabet, D. Montes, M.E. Moreno-Raya, A. Moya, R. Mundt, E. Nagel, V. Naranjo, L. Nortmann, G. Nowak, A. Ofir, R. Oreiro, E. Pallé, J. Panduro, J. Pascual, V.M. Passegger, A. Pavlov, S. Pedraz, A. Pérez-Calpena, D. Pérez Medialdea, M. Perger, M.A.C. Perryman, M. Pluto, O. Rabaza, A. Ramón, R. Rebolo, P. Redondo, S. Reffert, S. Reinhart, P. Rhode, H.W. Rix, F. Rodler, E. Rodríguez, C. RodríguezLópez, A. Rodríguez Trinidad, R.R. Rohloff, A. Rosich, S. Sadegi, E. Sánchez-Blanco, M.A. Sánchez Carrasco, A. Sánchez-López, J. Sanz-Forcada, P. Sarkis, L.F. Sarmiento, S. Schäfer, J.H.M.M. Schmitt, J. Schiller, A. Schweitzer, E. Solano, O. Stahl, J.B.P. Strachan, J. Stürmer, J.C. Suárez, H.M. Tabernero, M. Tala, T. Trifonov, S.M. Tulloch, R.G. Ulbrich, G. Veredas, J.I. Vico Linares, F. Vilardell, K. Wagner, J. Winkler, V. Wolthoff, W. Xu, F. Yan, M.R. Zapatero Osorio, The CARMENES search for exoplanets around M dwarfs. High-resolution optical and near-infrared spectroscopy of 324 survey stars. Astron. Astrophys. 612, A49 (2018)

I. Ribas, E. Bolmont, F. Selsis, A. Reiners, J. Leconte, S.N. Raymond, S.G. Engle, E.F. Guinan, J. Morin, M. Turbet, F. Forget, G. Anglada-Escudé, The habitability of Proxima Centauri b. I. Irradiation, rotation and volatile inventory from formation to the present. Astron. Astrophys. 596, A111 (2016)

G.H. Rieke, G.S. Wright, T. Böker, J. Bouwman, L. Colina, A. Glasse, K.D. Gordon, T.P. Greene, M. Güdel, T. Henning, K. Justtanont, P.O. Lagage, M.E. Meixner, H.U. Nørgaard-Nielsen, T.P. Ray, M.E. Ressler, E.F. van Dishoeck, C. Waelkens, The mid-infrared instrument for the James Webb Space Telescope, I: introduction. Publ. Astron. Soc. Pac. 127(953), 584 (2015)

F. Rodler, M. López-Morales, Feasibility studies for the detection of $\mathrm{O}_{2}$ in an Earth-like exoplanet. Astrophys. J. 781(1), 54 (2014)

R.M. Roettenbacher, S.R. Kane, The stellar activity of TRAPPIST-1 and consequences for the planetary atmospheres. Astrophys. J. 851, 77 (2017)

R.A. Rossiter, On the detection of an effect of rotation during eclipse in the velocity of the brigher component of beta Lyrae, and on the constancy of velocity of this system. Astrophys. J. 60, 15-21 (1924)

S. Rugheimer, L. Kaltenegger, A. Segura, J. Linsky, S. Mohanty, Effect of UV radiation on the spectral fingerprints of Earth-like planets orbiting M stars. Astrophys. J. 809(1), 57 (2015a)

S. Rugheimer, A. Segura, L. Kaltenegger, D. Sasselov, UV surface environment of Earth-like planets orbiting FGKM stars through geological evolution. Astrophys. J. 806(1), 137 (2015b)

C. Sagan, C. Chyba, The early faint sun paradox: organic shielding of ultraviolet-labile greenhouse gases. Science 276, 1217-1221 (1997)

D. Saumon, G. Chabrier, H.M. van Horn, An equation of state for low-mass stars and giant planets. Astrophys. J. 99, 713 (1995)

L. Schaefer, R.D. Wordsworth, Z. Berta-Thompson, D. Sasselov, Predictions of the atmospheric composition of GJ 1132b. Astrophys. J. 829(2), 63 (2016)

A.C. Schneider, E.L. Shkolnik, HAZMAT. III. The UV evolution of mid- to late-M stars with GALEX. Astron. J. 155(3), 122 (2018)

J. Schneider, C. Dedieu, P. Le Sidaner, R. Savalle, I. Zolotukhin, Defining and cataloging exoplanets: the exoplanet.eu database. Astron. Astrophys. 532, A79 (2011)

D. Schoonenberg, B. Liu, C.W. Ormel, C. Dorn, Pebble-driven planet formation for TRAPPIST-1 and other compact systems. Astron. Astrophys. 627, A149 (2019)

S. Seager, M. Kuchner, C.A. Hier-Majumder, B. Militzer, Mass-radius relationships for solid exoplanets. Astrophys. J. 669, 1279-1297 (2007)

A. Segura, L.M. Walkowicz, V. Meadows, J. Kasting, S. Hawley, The effect of a strong stellar flare on the atmospheric chemistry of an Earth-like planet orbiting an M dwarf. Astrobiology 10(7), 751-771 (2010)

F. Selsis, R.D. Wordsworth, F. Forget, Thermal phase curves of nontransiting terrestrial exoplanets. I. Characterizing atmospheres. Astron. Astrophys. 532, A1 (2011)

D.B. Serindag, I.A.G. Snellen, Testing the detectability of extraterrestrial $\mathrm{O}_{2}$ with the extremely large telescopes using real data with real noise. Astrophys. J. Lett. 871(1), L7 (2019)

K. Shibata, T. Magara, Solar flares: magnetohydrodynamic processes. Living Rev. Sol. Phys. 8(1), 6 (2011)

I.A.G. Snellen, R.J. de Kok, R. le Poole, M. Brogi, J. Birkby, Finding extraterrestrial life using ground-based high-dispersion spectroscopy. Astrophys. J. 764(2), 182 (2013)

D. Tamayo, H. Rein, C. Petrovich, N. Murray, Convergent migration renders TRAPPIST-1 long-lived. Astrophys. J. Lett. 840(2), L19 (2017) 
C. Terquem, J.C.B. Papaloizou, Migration and the formation of systems of hot super-Earths and Neptunes. Astrophys. J. 654(2), 1110-1120 (2007)

F. Tian, Thermal escape from super Earth atmospheres in the habitable zones of M stars. Astrophys. J. 703(1), 905-909 (2009)

F. Tian, History of water loss and atmospheric $\mathrm{O}_{2}$ buildup on rocky exoplanets near $\mathrm{M}$ dwarfs. Earth Planet. Sci. Lett. 432, 126-132 (2015)

F. Tian, S. Ida, Water contents of Earth-mass planets around M dwarfs. Nat. Geosci. 8, 177-180 (2015)

F. Tian, J.F. Kasting, H.-L. Liu, R.G. Roble, Hydrodynamic planetary thermosphere model: 1. Response of the Earth's thermosphere to extreme solar EUV conditions and the significance of adiabatic cooling. J. Geophys. Res., Planets 113(E5), E05008 (2008a)

F. Tian, S.C. Solomon, L. Qian, J. Lei, R.G. Roble, Hydrodynamic planetary thermosphere model: 2. Coupling of an electron transport/energy deposition model. J. Geophys. Res., Planets 113(E7), E07005 (2008b)

F. Tian, J.F. Kasting, K. Zahnle, Revisiting HCN formation in Earth's early atmosphere. Earth Planet. Sci. Lett. 308, 417-423 (2011)

M. Turbet, J. Leconte, F. Selsis, E. Bolmont, F. Forget, I. Ribas, S.N. Raymond, G. Anglada-Escudé, The habitability of Proxima Centauri b. II. Possible climates and observability. Astron. Astrophys. 596, A112 (2016)

M. Turbet, E. Bolmont, J. Leconte, F. Forget, F. Selsis, G. Tobie, A. Caldas, J. Naar, M. Gillon, Modeling climate diversity, tidal dynamics and the fate of volatiles on TRAPPIST-1 planets. Astron. Astrophys. 612, A86 (2018)

M. Turbet, D. Ehrenreich, C. Lovis, E. Bolmont, T. Fauchez, The runaway greenhouse radius inflation effect-an observational diagnostic to probe water on Earth-sized planets and test the habitable zone concept. Astron. Astrophys. 628, A12 (2019a)

M. Turbet, H. Tran, O. Pirali, F. Forget, C. Boulet, J.-M. Hartmann, Far infrared measurements of absorptions by $\mathrm{CH}_{4}+\mathrm{CO}_{2}$ and $\mathrm{H}_{2}+\mathrm{CO}_{2}$ mixtures and implications for greenhouse warming on early Mars. Icarus 321, 189-199 (2019b)

M. Turbet, E. Bolmont, D. Ehrenreich, P. Gratier, J. Leconte, F. Selsis, N. Hara, C. Lovis, Revised mass-radius relationships for water-rich rocky planets more irradiated than the runaway greenhouse limit. Astron. Astrophys. 638, A41 (2020a)

M. Turbet, C. Boulet, T. Karman, Measurements and semi-empirical calculations of $\mathrm{CO} 2+\mathrm{CH} 4$ and $\mathrm{CO} 2+\mathrm{H} 2$ collision-induced absorption across a wide range of wavelengths and temperatures. Application for the prediction of early Mars surface temperature. Icarus 346, 113762 (2020b)

C.T. Unterborn, S.J. Desch, N.R. Hinkel, A. Lorenzo, Inward migration of the TRAPPIST-1 planets as inferred from their water-rich compositions. Nat. Astron. 2, 297-302 (2018)

V. Van Grootel, C.S. Fernandes, M. Gillon, E. Jehin, J. Manfroid, R. Scuflaire, A.J. Burgasser, K. Barkaoui, Z. Benkhaldoun, A. Burdanov, L. Delrez, B.-O. Demory, J. de Wit, D. Queloz, A.H.M.J. Triaud, Stellar parameters for Trappist-1. Astrophys. J. 853, 30 (2018)

K. Vida, Z. Kővári, A. Pál, K. Oláh, L. Kriskovics, Frequent flaring in the TRAPPIST-1 system-unsuited for life? Astrophys. J. 841, 124 (2017)

A. Vidal-Madjar, E.A. des Lecavelier, J.-M. Désert, G.E. Ballester, R. Ferlet, G. Hébrard, M. Mayor, An extended upper atmosphere around the extrasolar planet HD209458b. Nature 422, 143-146 (2003)

A.M. Vinson, B.M.S. Hansen, On the spin states of habitable zone exoplanets around M dwarfs: the effect of a near-resonant companion. Mon. Not. R. Astron. Soc. 472(3), 3217-3229 (2017)

A.M. Vinson, D. Tamayo, B.M.S. Hansen, The chaotic nature of TRAPPIST-1 planetary spin states. Mon. Not. R. Astron. Soc. 488(4), 5739-5747 (2019)

H.R. Wakeford, N.K. Lewis, J. Fowler, G. Bruno, T.J. Wilson, S.E. Moran, J. Valenti, N.E. Batalha, J. Filippazzo, V. Bourrier, S.M. Hörst, S.M. Lederer, J. de Wit, Disentangling the planet from the star in late-type M dwarfs: a case study of TRAPPIST-1g. Astron. J. 157(1), 11 (2019)

A. Way M. J., D. Del Genio, Venusian habitable climate scenarios: modeling Venus through time and applications to slowly rotating Venus-like exoplanets (2020). ArXiv e-prints, arXiv:2003.05704

M.J. Way, I. Aleinov, D.S. Amundsen, M.A. Chand ler, T.L. Clune, A.D. Del Genio, Y. Fujii, M. Kelley, N.Y. Kiang, L. Sohl, K. Tsigaridis, Resolving orbital and climate keys of Earth and extraterrestrial environments with dynamics (ROCKE-3D) 1.0: a general circulation model for simulating the climates of rocky planets. Astrophys. J. 231(1), 12 (2017)

P.J. Wheatley, T. Louden, V. Bourrier, D. Ehrenreich, M. Gillon, Strong XUV irradiation of the Earth-sized exoplanets orbiting the ultracool dwarf TRAPPIST-1. Mon. Not. R. Astron. Soc. 465, L74-L78 (2017)

F. Wildi, N. Blind, V. Reshetov, O. Hernandez, L. Genolet, U. Conod, M. Sordet, A. Segovilla, J.L. Rasilla, D. Brousseau, S. Thibault, B. Delabre, T. Bandy, M. Sarajlic, A. Cabral, S. Bovay, P. Vallée, F. Bouchy, R. Doyon, E. Artigau, F. Pepe, J. Hagelberg, C. Melo, X. Delfosse, P. Figueira, N.C. Santos, J.I. González Hernández, J.R. de Medeiros, R. Rebolo, C. Broeg, W. Benz, I. Boisse, L. Malo, U. Käufl, L. Sad- 
dlemyer, NIRPS: an adaptive-optics assisted radial velocity spectrograph to chase exoplanets around M-stars, in Proceedings of the SPIE. Society of Photo-Optical Instrumentation Engineers (SPIE) Conference Series, vol. 10400 (2017), p. 1040018

E.T. Wolf, Assessing the habitability of the TRAPPIST-1 system using a 3D climate model. Astrophys. J. Lett. 839, L1 (2017)

E.T. Wolf, Erratum: assessing the habitability of the TRAPPIST-1 system using a 3D climate model. Astrophys. J. Lett. 855(1), L14 (2018)

E.T. Wolf, O.B. Toon, Fractal organic hazes provided an ultraviolet shield for early Earth. Science 328, 1266 (2010)

E.T. Wolf, O.B. Toon, The evolution of habitable climates under the brightening Sun. J. Geophys. Res., Atmos. 120, 5775-5794 (2015)

R. Wordsworth, Atmospheric heat redistribution and collapse on tidally locked rocky planets. Astrophys. J. $\mathbf{8 0 6}(2), 180$ (2015)

R.D. Wordsworth, F. Forget, F. Selsis, E. Millour, B. Charnay, J.-B. Madeleine, Gliese 581d is the first discovered terrestrial-mass exoplanet in the habitable zone. Astrophys. J. Lett. 733, L48 (2011)

R. Wordsworth, Y. Kalugina, S. Lokshtanov, A. Vigasin, B. Ehlmann, J. Head, C. Sanders, H. Wang, Transient reducing greenhouse warming on early Mars. Geophys. Res. Lett. 44, 665-671 (2017)

R.D. Wordsworth, L.K. Schaefer, R.A. Fischer, Redox evolution via gravitational differentiation on low-mass planets: implications for abiotic oxygen, water loss, and habitability. Astron. J. 155, 195 (2018)

J.T. Wright, Planet-planet tides in the TRAPPIST-1 system. Res. Notes Am. Astron. Soc. 2(3), 175 (2018)

F. Wunderlich, M. Godolt, J.L. Grenfell, S. Städt, A.M.S. Smith, S. Gebauer, F. Schreier, P. Hedelt, H. Rauer, Detectability of atmospheric features of Earth-like planets in the habitable zone around M dwarfs. Astron. Astrophys. 624, A49 (2019)

J. Yang, N.B. Cowan, D.S. Abbot, Stabilizing cloud feedback dramatically expands the habitable zone of tidally locked planets. Astrophys. J. Lett. 771, L45 (2013)

J. Yang, G. Boué, D.C. Fabrycky, D.S. Abbot, Strong dependence of the inner edge of the habitable zone on planetary rotation rate. Astrophys. J. Lett. 787, L2 (2014a)

J. Yang, Y. Liu, Y. Hu, D.S. Abbot, Water trapping on tidally locked terrestrial planets requires special conditions. Astrophys. J. Lett. 796, L22 (2014b)

Y.L. Yung, M. Allen, J.P. Pinto, Photochemistry of the atmosphere of Titan-comparison between model and observations. Astrophys. J. Suppl. Ser. 55, 465-506 (1984)

L. Zeng, D.D. Sasselov, S.B. Jacobsen, Mass-radius relation for rocky planets based on PREM. Astrophys. J. 819, 127 (2016)

Z. Zhang, Y. Zhou, B.V. Rackham, D. Apai, The near-infrared transmission spectra of TRAPPIST-1 planets b, c, d, e, f, and g and stellar contamination in multi-epoch transit spectra. Astron. J. 156(4), 178 (2018) 